\title{
ON SCATTERING FOR THE QUINTIC DEFOCUSING NONLINEAR SCHRÖDINGER EQUATION ON $\mathbb{R} \times \mathbb{T}^{2}$.
}

\author{
ZAHER HANI AND BENOIT PAUSADER
}

\begin{abstract}
We consider the problem of large data scattering for the quintic nonlinear Schrödinger equation on $\mathbb{R} \times \mathbb{T}^{2}$. This equation is critical both at the level of energy and mass. Most notably, we exhibit a new type of profile (a "large scale profile") that controls the asymptotic behavior of the solutions.
\end{abstract}

\section{INTRODUCTION}

The purpose of this manuscript is to study the asymptotic behavior of the defocusing quintic nonlinear Schrödinger equation on $\mathbb{R} \times \mathbb{T}^{2}$ given by

$$
\left(i \partial_{t}+\Delta_{\mathbb{R} \times \mathbb{T}^{2}}\right) u=|u|^{4} u, \quad u(t=0)=u_{0} \in H^{1}\left(\mathbb{R} \times \mathbb{T}^{2}\right)
$$

Our main motivation is to better understand the broad question of the effect of the geometry of the domain on the asymptotic behavior of large solutions to nonlinear dispersive equations. While scattering holds for the quintic equation on $\mathbb{R}^{3}$, it is not expected to hold (apart from trivial cases) on $\mathbb{T}^{3}$ (cf. Appendix to [19]). As we will argue below, the situation on $\mathbb{R} \times \mathbb{T}^{2}$ seems to be a borderline case for this question.

The study of solutions of the nonlinear Schrödinger equation on compact or partially compact domains has been the subject of many works, dating back at least to 11 and then systematically developed by Bourgain 8, 9] on tori and Burq-Gérard-Tzvetkov [12, 13, 14] on compact manifolds, with recent key developments in the energy-critical setting following the work of Herr-Tataru-Tzvetkov [31, 32, 30, and extended to global existence in Ionescu-Pausader [34, 35]. We also refer to [6, 7, 24, 26, 29, 33, 49. and especially to 4, 55 for previous works on the relation between scattering and geometry. Such equations have also been extensively studied in applied sciences on various backgrounds. While we will not attempt to make any fairly exhaustive list of those works, we should point out that their study on "wave-guide" manifolds like $\mathbb{R} \times \mathbb{T}^{2}$ seems to be of particular interest especially in non-linear optics of telecommunications [47, 43, 22,

The studies on global wellposedness for energy critical and subcritical equation 3 so far seem to point to the absence of any geometric obstruction to global existence. Indeed in the cases known so far, the solutions to the defocusing problem all extend globally. In contrast, it is clear that the geometry influences strongly the asymptotic dynamics of solutions but the precise relationship remains poorly understood.

The first author was supported in part by a Simons Postdoctoral Fellowship. The second author was supported in part by NSF grant DMS-1069243.

${ }^{1}$ Since our main goal is to attack the large-data long-time theory, the regularity of our initial data is dictated by the conservation laws. For large data global regularity and scattering, considering data in $H^{1}$ or in any smoother space is effectively equivalent.

${ }^{2}$ That is when the potential part of the energy can be bounded in terms of the kinetic part. We remark that the energysupercritical case (the complementary case which we do not discuss) is completely unknown, even on Euclidean spaces $\mathbb{R}^{d}$, except for some ill-posedness results [2, 13, 54, or some results on the "barely supercritical" case [46] 52. 
A first step in this direction is to explore when one can obtain the simplest asymptotic behavior, namely scattering, which means that all nonlinear solutions asymptotically resemble linear solutions. From the heuristic that linear solutions with frequency $\sim N$ initially localized around the origin will disperse at time $t$ in the ball of radius $\sim N t$, one can hope that scattering is partly determined by the asymptotic volume growth of balls with respect to their radius. In fact, if

$$
V(r):=\inf _{q \in M^{d}}\left\{\operatorname{Vol}_{M^{d}}(B(q, r))\right\} \sim_{r \rightarrow \infty} r^{g},
$$

then one would expect that linear solutions decay at a rate $\sim t^{-g / 2}$ and based on the Euclidean theory on $\mathbb{R}^{g}$, the equation

$$
\left(i \partial_{t}+\Delta_{M^{d}}\right) u=|u|^{p-1} u, \quad u(0) \in H^{1}\left(M^{d}\right)
$$

would scatter in the range $1+4 / g \leq p \leq 1+4 /(d-2)$, while one might expect more exotic behavior, at least when $p \leq 1+2 / g$. Note that this heuristics is consistent with the results in [4].

We don't know whether such a simple picture is accurate but testing this hypothesis motivated us to study the asymptotic behavior for (1.1) in the case $g=1$ and $d=3$, which seems to be the hardest case that can be addressed in light of the recent developments in 31, 21. Indeed, as we will argue later, this problem is both mass-critical and energy-critical $(1+4 / g=1+4 /(d-2)=5)$.

Our two main results tend to confirm the picture above about scattering, at least in the case of quotients of Euclidean spaces and insofar as one can parallel the case $p=1+4 / g$ and the mass-critical problem in $\mathbb{R}^{g}$. The first result asserts that small initial data lead to solutions which are global and scatter.

Theorem 1.1. There exists $\delta>0$ such that any initial data $u_{0} \in H^{1}\left(\mathbb{R} \times \mathbb{T}^{2}\right)$ satisfying

$$
\left\|u_{0}\right\|_{H^{1}\left(\mathbb{R} \times \mathbb{T}^{2}\right)} \leq \delta
$$

leads to a unique global solution $u \in X_{c}^{1}(\mathbb{R})$ of (1.1) which scatters in the sense that there exists $v^{ \pm} \in$ $H^{1}\left(\mathbb{R} \times \mathbb{T}^{2}\right)$ such that

$$
\left\|u(t)-e^{i t \Delta_{\mathbb{R} \times \mathbb{T}^{2}}} v^{ \pm}\right\|_{H^{1}\left(\mathbb{R} \times \mathbb{T}^{2}\right)} \rightarrow 0 \quad \text { as } \quad t \rightarrow \pm \infty .
$$

The uniqueness space $X_{c}^{1} \subset C_{t}\left(\mathbb{R}: H^{1}\left(\mathbb{R} \times \mathbb{T}^{2}\right)\right)$ was essentially introduced by Herr-Tataru-Tzvetkov [31]. The main novelty here is the scattering statement on a manifold with such little volume (and so many trapped geodesics $3^{3}$ ). Using the time-reversal symmetry, a similar statement holds for negative times as well. One key fact about Theorem 1.1 is that it requires only a control provided by the conserved mass and energy of the solution defined respectively by

$$
M(u):=\|u(0)\|_{L^{2}\left(\mathbb{R} \times \mathbb{T}^{2}\right)}^{2}, \quad E(u):=\frac{1}{2}\|\nabla u(0)\|_{L^{2}\left(\mathbb{R} \times \mathbb{T}^{2}\right)}^{2}+\frac{1}{6}\|u(0)\|_{L^{6}\left(\mathbb{R} \times \mathbb{T}^{2}\right)}^{6} .
$$

We will refer to the quantity $L(u):=\frac{1}{2} M(u)+E(u)$ as the "full energy" of $u$. We should mention the work of Tzvetkov-Visciglia [55] for a previous scattering statement involving a norm which is not bounded by the full energy. The main advantage of having a control at the level of a conserved quantity is that one can then hope to extend a small data result to a global result. This is precisely the question we tackle in the main part of this paper.

In order to extend our analysis to large data, we use a method formalized in Kenig-Merle [36] following previous works on critical nonlinear dispersive equations [3, 10, 18, 38, see also [41, 20. One key ingredient is a linear and nonlinear profile decomposition for solutions with bounded energy. The so-called profiles correpond to sequences of solutions exhibiting an extreme behavior (in fact a defect of compactness) and

\footnotetext{
${ }^{3}$ The presence of trapped geodesics is known to have nontrivial effects on the linear flow and could be expected to also affect the asymptotic behavior of nonlinear solutions.
} 
possibly "leaving" the geometry. It is there that the "energy-critical" and "mass-critical" nature of our equation become manifest.

In order to understand the appearance of the profiles, one can argue as follows: in view of the scaling invariance of (1.1) under

$$
\mathbb{R}_{x} \times \mathbb{T}_{y}^{2} \rightarrow M_{\lambda}:=\mathbb{R}_{x} \times\left(\lambda^{-1} \mathbb{T}^{2}\right)_{y}, \quad u \rightarrow \tilde{u}(x, y, t)=\lambda^{1 / 2} u\left(\lambda x, \lambda y, \lambda^{2} t\right),
$$

a uniform control on the solutions on $M_{1}$ in terms of their full energy yields a control on solutions on $M_{\lambda}$ uniformly in $\lambda$. Therefore, we would expect a solution initially at "scale one" on $M_{\lambda}$ to remain so. We then observe the two natural scaling limits:

i) Small-scale limit: when $\lambda \rightarrow 0$, a "scale-one" solution on $M_{\lambda}$ should not "sense" the distinction between $M_{\lambda}$ and $\mathbb{R}^{3}$ and should therefore behave as a solution to the energy-critical NLS on $\mathbb{R}^{3}$. This corresponds in $M_{1}$ to solutions with initial data

$$
u^{\lambda}(x, y, 0)=\lambda^{-1 / 2} \phi\left(\lambda^{-1}(x, y)\right), \quad \phi \in C_{c}^{\infty}\left(\mathbb{R}^{3}\right), \lambda \rightarrow 0 .
$$

We call such profiles Euclidean profiles. Their appearance is a manifestation of the energy-critical nature of the nonlinearity .

ii) Large-scale limit: when $\lambda \rightarrow+\infty$ the manifold $M_{\lambda}$ becomes thinner and thinner and "resembles $\mathbb{R}^{\prime}$. It is then tempting to guess that scale-one solutions on $M_{\lambda}$ will evolve, on time-scales of size $\lambda^{2}$, as the solutions to the quintic (mass-critical) nonlinear Schrödinger equation on $\mathbb{R}$ :

$$
\left(i \partial_{t}+\partial_{x x}\right) u=|u|^{4} u, \quad u(0) \in H^{1}(\mathbb{R})
$$

and this is indeed the case when the initial data is constant in the compact $(y)$ variable. However, the general situation is more complicated as different Fourier modes will interact in a nontrivial manner and this "fast dynamics" has a profound effect on the slow dynamics coming from (1.5). Indeed the rigorous analysis of this "multi-scale dynamics" is one of the main component of this paper.

These solutions on $M_{\lambda}$ correspond to what we call the "large-scale profiles" on $M_{1}$ which are initial data given by:

$$
u^{\lambda}(x, y, 0)=\lambda^{-1 / 2} \phi\left(\lambda^{-1} x, y\right), \quad \phi \in C_{c}^{\infty}\left(\mathbb{R} \times \mathbb{T}^{2}\right), \lambda \rightarrow+\infty
$$

and their appearance seems to be a manifestation of the mass-critical nature of the equation. We will argue below that these solutions should follow the flow associated to a more general flow than that of (1.5), namely the one given by quintic resonant system on $\mathbb{R}$ that we now describe.

The quintic resonant system is given by

$$
\begin{aligned}
\left(i \partial_{t}+\partial_{x x}\right) u_{j}= & \sum_{\mathcal{R}(j)} u_{j_{1}} \overline{u_{j_{2}}} u_{j_{3}} \overline{u_{j_{4}}} u_{j_{5}} \quad j \in \mathbb{Z}^{2} \\
\mathcal{R}(j)= & \left\{\left(j_{1}, j_{2}, j_{3}, j_{4}, j_{5}\right) \in\left(\mathbb{Z}^{2}\right)^{5}: j_{1}-j_{2}+j_{3}-j_{4}+j_{5}=j\right. \text { and } \\
& \left.\left|j_{1}\right|^{2}-\left|j_{2}\right|^{2}+\left|j_{3}\right|^{2}-\left|j_{4}\right|^{2}+\left|j_{5}\right|^{2}=|j|^{2}\right\}
\end{aligned}
$$

with unknown $\vec{u}=\left\{u_{j}\right\}_{j \in \mathbb{Z}^{2}}$, where $u_{j}: \mathbb{R}_{x} \times \mathbb{R}_{t} \rightarrow \mathbb{C}$. In the special case when $u_{j}=0$ for $j \neq 0$, we recover (1.5), but in general, this is a new equation. Similar finite or infinite systems of nonlinear Schrödinger equations arise independently in the study of nonlinear optics in waveguides and are the object of several previous studie:5] (see e.g. [17, 25, 44], the books [1, 48] and references therein).

\footnotetext{
${ }^{4}$ In the sense that it corresponds to the critical exponent with respect to Sobolev embedding; in other words, with the notations in (1.2), $p=1+4 /(d-2)$.

${ }^{5}$ They sometimes go by the name of "vector nonlinear Schrödinger equation" or VNLS.
} 
As we show in an appendix, the system (1.6) is Hamiltonian, has a nice local theory and retains many properties of (1.5). In view of this and of the result of Dodson [21, it seems reasonable to formulate the following conjecture:

Conjecture 1.2. Let $E \in(0, \infty)$. For any smooth initial data $\vec{u}_{0}$ satisfying:

$$
E_{l s}\left(\vec{u}_{0}\right):=\frac{1}{2} \sum_{j \in \mathbb{Z}}\langle j\rangle^{2}\left\|u_{0, j}\right\|_{L^{2}(\mathbb{R})}^{2} \leq E
$$

there exists a global solution of (1.6), $\vec{u}(t), \vec{u}(t=0)=\vec{u}_{0}$ with conserved $E_{l s}(\vec{u}(t))=E_{l s}\left(\vec{u}_{0}\right)$ satisfying:

$$
\|\vec{u}\|_{\vec{W}}^{2}:=\sum_{j \in \mathbb{Z}}\langle j\rangle^{2}\left\|u_{j}\right\|_{L_{x, t}^{6}\left(\mathbb{R}_{x} \times \mathbb{R}_{t}\right)}^{2} \leq \Lambda_{l s}\left(E_{l s}\left(\vec{u}_{0}\right)\right) .
$$

for some finite non-decreasing function $\Lambda_{l s}(E)$.

We can now give the main result of this paper which asserts large data scattering for (1.1) conditioned on Conjecture 1.2

Theorem 1.3. Assume that Conjecture 1.2 holds for all $E \leq E_{\text {max }}^{l s}$, then any initial data $u_{0} \in H^{1}\left(\mathbb{R} \times \mathbb{T}^{2}\right)$ satisfying

$$
L\left(u_{0}\right)=\int_{\mathbb{R} \times \mathbb{T}^{2}}\left\{\frac{1}{2}\left|u_{0}\right|^{2}+\frac{1}{2}\left|\nabla u_{0}\right|^{2}+\frac{1}{6}\left|u_{0}\right|^{6}\right\} d x \leq E_{\text {max }}^{l s}
$$

leads to a solution $u \in X_{c}^{1}(\mathbb{R})$ which is global, and scatters in the sense that there exists $v^{ \pm} \in H^{1}\left(\mathbb{R} \times \mathbb{T}^{2}\right)$ such that (1.3) holds. In particular, if $E_{\max }^{l s}=+\infty$, then all solutions of (1.1) with finite energy and mass scatter.

Before we go into the details of the proof, a few remarks about the above theorem are in order. First, we should point out that the global regularity part holds for all solutions of finite energy, unconditional on Conjecture 1.2. Second, as a consequence of the local theory for the system (1.6), one has that Conjecture 1.2 holds below a nonzero threshold $E_{\max }^{l s}>0$, so Theorem 1.3 is non-empty and actually strengthens Theorem 1.1. Actually, by simple modifications of the proof, one can find regimes with large mass (for example using the main result in 21]) where one can obtain nontrivial large data that scatter. Another point worth mentioning is that while Theorem 1.3 is stated as an implication, it is actually an equivalence as it is easy to see that one can reverse the analysis needed to understand the behavior of large-scale profile initial data for (1.1) in order to control general solutions of (1.6) and prove Conjecture 1.2 assuming that Theorem 1.3 holds (cf. Appendix). Finally, we note that the full resolution of Conjecture 1.2 seems to require considerable additional work that is completely independent of the analysis on $\mathbb{R} \times \mathbb{T}^{2}$, so we choose to leave it for a later work.

The proof of Theorem 1.3 has a fairly standard skeleton based on the Kenig-Merle machinery [36 on one hand, and the recent works devoted to understanding inhomogeneous critical equations 33, 34, 35, 39, 40, on the other. Nonetheless, the global geometry, the two levels of criticality (mass and energy), and the emergence of large-scale profiles require some novel ideas and nonstandard adaptations. Most notably: i) proving good global Strichartz estimates not only to prove Theorem 1.1 but also to obtain an $L^{2}$-profile decomposition suitable for the large data theory, ii) the analysis of the large-scale profile initial data that appear in the profile decomposition, understanding their "two time-scale" behavior in terms of the quintic system (1.6) via a normal form transformation, and iii) a final nonlinear profile recomposition similar to that in [34] but with many more cases. We elaborate briefly on those three points: 
1.1. Global Strichartz estimates. While local-in-time Strichartz estimates are sufficient for the purposes of local existence and even global regularity [34, 35, they are not useful to obtain information about the asymptotic behavior, even for small data. On the other hand, in order to prove scattering with critical control (at the level of $H^{1}$ ), one cannot afford to work with Strichartz estimates that lose too many derivatives. These two limitations (good global-in-time integrability and derivative loss) encapsulate the main difficulties at this level.

By using the Strichartz estimates on $\mathbb{R}$ and Sobolev embedding, one can obtain global-in-time bounds with optimal scaling such as:

$$
\left\|e^{i t \Delta_{\mathbb{R} \times \mathbb{T}^{2}}} u_{0}\right\|_{L_{x, y, t}^{6}\left(\mathbb{R} \times \mathbb{T}^{2} \times \mathbb{R}\right)} \lesssim\left\|\langle\nabla\rangle^{\frac{2}{3}} u_{0}\right\|_{L_{x, y}^{2}\left(\mathbb{R} \times \mathbb{T}^{2}\right)} .
$$

However, this derivative loss of $2 / 3$ derivatives does not allow for a local theory at the level of $H^{1}$, which seems to require estimates with less than $1 / 2$ derivatives lost. On the other hand, one can also easily obtain local-in-time Strichartz estimates with satisfactory derivative loss from the corresponding estimates on $\mathbb{T}^{2}$, but with no obvious way to extend them globally. In addition, application of the HardyLittlewood circle method as in [8, 34] requires the use of the same $L^{p}$ norms in $x, y, t$ and no such $p$ can give both sufficiently good derivative loss and global-in-time integrability.

This suggests using norms that distinguish between local-in-time and global-in-time integrability. We are thus lead to the following Strichartz estimates:

$$
\left\|e^{i t \Delta_{\mathbb{R} \times \mathbb{T}^{2}}} u_{0}\right\|_{l_{\gamma}^{q} L_{x, y, t}^{r}\left(\mathbb{R} \times \mathbb{T}^{2} \times[\gamma, \gamma+1]\right)} \lesssim\left\|\langle\nabla\rangle^{\frac{3}{2}-\frac{5}{r}} u_{0}\right\|_{L_{x, y}^{2}\left(\mathbb{R} \times \mathbb{T}^{2}\right)}, \quad \frac{2}{q}+\frac{1}{r}=\frac{1}{2}, \quad 4<q, r<+\infty,
$$

where we take the $l^{q}$ sum in $\gamma$ of the $L_{x, y, t}^{p}\left(\mathbb{R} \times \mathbb{T}^{2} \times[\gamma, \gamma+1]\right)$ norm on the left hand side. Notice that the condition on $q$ is exactly the $1 d$ Schrödinger admissibility condition on $\mathbb{R}$ whereas the derivative loss of $3 / 2-5 / r$ is exactly that on $\mathbb{T}^{3}$ (or $\mathbb{R}^{3}$ ). The proof of this estimate follows a $T T^{*}$ argument that allows to decompose the relevant inner product into a diagonal part (the solution at time $t$ interacting with itself at a similar time) and a non-diagonal part (the solution at time $t$ interacting with itself at time $s$ with $|s-t| \gg 1$ ). The diagonal component leads to the loss of derivatives, but gives a contribution that has better time-integrability (like $l_{\gamma}^{2}$ ). The non-diagonal part loses fewer derivatives but forces the abovementioned slower $l_{\gamma}^{q}$ time-integrability and requires a nontrivial adaptation of the Hardy-Littlewood circle method in a way that incorporates the decay in $|s-t|$ coming from the $\mathbb{R}$ direction. The fact that we can decouple those two difficulties (loss of derivatives and slow integrability) is crucial in obtaining a good profile decomposition in this mass-critical context.

1.2. Large-scale profiles and the resonant quintic system. The treatment of the large-scale profiles alluded to in our previous heuristic and formalized using the profile decomposition leads to the quintic resonant system (1.6). This system is not completely unexpected as it can be derived from (1.1) by removing all non-resonant interactions between the periodic Fourier modes of $u$. In general, one would expect non-resonant interactions to have important large-time contributions especially for large data. However, in the special limit given by those large-scale profile initial data, one can integrate out the non-resonant dynamics by using a normal form transformation and this yields (1.6).

Among the three types of sequences appearing in the profile decomposition, the large-scale profiles correspond to sequences of solutions of (1.1) with initial data roughly of the form:

$$
\psi_{k}(x, y)=M_{k}^{1 / 2} \psi\left(M_{k} x, y\right), \quad \text { with } \psi \in C_{c}^{\infty}\left(\mathbb{R} \times \mathbb{T}^{2}\right), \text { and } M_{k} \rightarrow 0 .
$$

Predicting that this scaling is conserved leads one to consider $u(x, y, t)=M_{k}^{1 / 2} \tilde{u}\left(M_{k} x, y, t\right)$ where $\tilde{u}$ then satisfies the equation:

$$
\left(i \partial_{t}+\Delta_{\mathbb{T}^{2}}\right) \tilde{u}=M_{k}^{2}\left(|\tilde{u}|^{4} \tilde{u}-\partial_{x x} \tilde{u}\right), \quad \tilde{u}(0)=\psi .
$$


This suggests conjugating with respect to the corresponding semigroup $e^{i t \Delta_{\mathbb{T}^{2}}}$ and, after a rescaling, we see that $v$ satisfies an equation that is essentially:

$$
\left(i \partial_{t}+\partial_{x x}\right) v=\mathcal{N}(v)=\mathcal{O}\left(v^{5}\right)
$$

Since one would expect solutions of this equation to disperse after some large time $T_{0}$ (thanks to the dispersion of $e^{i t \Delta_{\mathbb{R}}}$ ), we only need to control the dynamics up to $T_{0}$. But over this time interval, the contribution of the non-resonant interactions is $O\left(T_{0} M_{k}^{2}\right)$ and hence can be ignored in the limit as $M_{k} \rightarrow 0$. Doing this leads to the quintic resonant system (1.6). The main conclusion of this analysis is that large-scale profiles admit the following "multi-scale" description 6

$$
u(x, y, t)=e^{i t \Delta_{\mathbb{T}^{2}}}\left[M_{k}^{\frac{1}{2}} v(X, y, T)\right], \quad X=M_{k} x, T=M_{k}^{2} t,
$$

where $v$ solves (1.6).

1.3. Profile recomposition and end of the proof. To end the argument, one needs to be able to write an approximate solution of (1.1) with initial data given as a sum of orthogonal profiles and dispersed initial data (i.e. one whose linear development is small in the relevant norm). A first guess for this approximate solution would be to sum the nonlinear profiles (solution of (1.1) with initial data given by the profiles) and the linear propagation of the perturbation. As in 34, this doesn't quite work because of terms that are linear in the perturbation. We follow [34 and focus on the second iterate of the Duhamel formula. While this does indeed give a good approximate solution it leaves us with proving that the newly defined perturbation is still small in the appropriate norm.

The main problem we face comes from perturbations that are concentrated at high frequencies and pointing only in the non-dispersive direction (near the zero frequency of $\mathcal{F}_{x}$ ). These solutions are close to concentrate on the trapped geodesics of $\mathbb{R} \times \mathbb{T}^{2}$ and they linger for a long time in compact regions of the manifold before they disperse, thereby interacting with solutions at larger scales for a long time. The redeeming features of such solutions are that i) their initial Fourier support makes them far from saturating the Strichartz estimates, so at least their linear development is small in a weak norm which makes their interaction with smaller scale profiles ineffective; ii) the qualitative fact that they are located in a small angular sector is conserved when they interact with larger scale nonlinear profiles, so the output of this interaction has similar properties. Implementing this requires introducing norms that are sensitive to the special Fourier support properties of the initial data and obtaining a good well-posedness theory to propagate the initial Fourier information effectively to later time:7.

The paper is organized as follows: in Section 2 we fix our notations and recall the function spaces we use; in Section 3 we prove the global Strichartz estimates we need for the rest of our work. Section 4 contains the local well-posedness and small-data scattering (Theorem 1.1) along with the associated stability theory. In Section [6 we obtain a good linear profile decomposition that leads us to the types of profiles we need to analyze, which is what we do in advance in Section 5 for the Euclidean and large-scale profiles. In Section 7 we prove the contradiction argument leading to Theorem 1.3. Finally, we develop the local existence theory for (1.6) in Section 8 .

\section{Notation and Function Spaces}

2.1. Notations. We write $A \lesssim B$ to signify that there is a constant $C>0$ such that $A \leq C B$. We write $A \simeq B$ when $A \lesssim B \lesssim A$. If the constant $C$ involved has some explicit dependency, we emphasize it by a subscript. Thus $A \lesssim_{u} B$ means that $A \leq C(u) B$ for some constant $C(u)$ depending on $u$. For $p \in \mathbb{N}^{n}$

\footnotetext{
${ }^{6}$ Indeed, this describes $u$ in terms of two "times" $t$ and $T$.

${ }^{7}$ Note that just putting the gradient of the highest frequency in $L^{2}$ loses any such information.
} 
a multi-index we denote by $\mathfrak{O}_{p_{1}, \ldots, p_{n}}\left(a_{1}, \ldots, a_{n}\right)$ a $|p|$-linear expression which is a product of $p_{1}$ terms which are either equal to $a_{1}$ or its complex conjugate $\bar{a}_{1}$ and similarly for $p_{j}, a_{j}, 2 \leq j \leq n$.

We label the coordinates on $\mathbb{R} \times \mathbb{T}^{2}$ as $\left(x, y_{1}, y_{2}\right) \in \mathbb{R} \times \mathbb{T} \times \mathbb{T}$. We fix $\eta^{1} \in C^{\infty}(\mathbb{R})$ such that $\eta^{1}(x)=1$ if $|x| \leq 1$ and $\eta^{1}(x)=0$ when $|x| \geq 2$. We also let $\eta^{3}(a, b, c)=\eta^{1}(a) \eta^{1}(b) \eta^{1}(c)$, so that for $x \in \mathbb{R}^{k}$ and $k \in\{1,3\}:$

$$
\eta_{\leq N}^{k}(x)=\eta^{k}(x / N), \quad \eta_{N}^{k}(x)=\eta_{\leq N}^{k}(x)-\eta_{\leq N / 2}^{k}(x), \quad \eta_{\geq N}^{k}(x)=1-\eta_{\leq N / 2}^{k}(x)
$$

For any dyadic number $N \geq 1$, we define the Littlewood-Paley projectors:

$$
P_{\leq N}:=\eta_{\leq N}^{3}(i \nabla), \quad P_{N}:=P_{\leq N}-P_{\leq N / 2} \text { for } N \geq 2 \text {, and } P_{1}:=P_{\leq 1} .
$$

Since we sometimes need to distinguish the regularity in the different directions, we similarly define $P_{\leq l}^{x}$ to be the Fourier multiplier associated to $\eta_{\leq M}^{1}\left(i \partial_{x}\right)$ and $P_{\leq N}^{y}$ to be the multiplier associated to $\eta_{\leq N}^{1}\left(i \partial_{y_{1}}\right) \eta_{\leq N}^{1}\left(i \partial_{y_{2}}\right)$. We define $P_{M}^{x}$ and $P_{M}^{y}$ similarly as before. We will also need the following angular frequency localization: for $\delta>0$, we define:

$$
\tilde{P}_{\delta}^{1}=\sum_{N \geq 1} P_{N} P_{\geq \delta N}^{x}
$$

In addition to the usual isotropic Sobolev spaces $H^{s}\left(\mathbb{R} \times \mathbb{T}^{2}\right)$, we will need non-isotropic versions. For $s_{1}, s_{2} \in \mathbb{R}$ we define:

$$
H^{s_{1}, s_{2}}\left(\mathbb{R} \times \mathbb{T}^{2}\right)=\left\{u: \mathbb{R} \times \mathbb{T}^{2} \rightarrow \mathbb{C}:\langle\xi\rangle^{s_{1}}\langle n\rangle^{s_{2}} \widehat{u}(\xi, n) \in L_{\xi, n}^{2}\left(\mathbb{R} \times \mathbb{Z}^{2}\right)\right\}
$$

We will be particularly interested in the space $H^{0,1}\left(\mathbb{R} \times \mathbb{T}^{2}\right)$. This is the set of functions $\psi \in L_{x, y}^{2}\left(\mathbb{R} \times \mathbb{T}^{2}\right)$ such that $\nabla_{y} \psi \in L_{x, y}^{2}\left(\mathbb{R} \times \mathbb{T}^{2}\right)$. We can also define a discrete analogue. For $\vec{\phi}=\left\{\phi_{p}\right\}_{p \in \mathbb{Z}^{2}}$ a sequence of real-variable functions, we let

$$
h^{s_{1}} H^{s_{2}}:=\left\{\vec{\phi}=\left\{\phi_{p}\right\}:\|\vec{\phi}\|_{h^{s_{1}} H^{s_{2}}}^{2}=\sum_{p \in \mathbb{Z}^{2}}\langle p\rangle^{2 s_{1}}\left\|\phi_{p}\right\|_{H^{s_{2}}}^{2}<+\infty\right\} .
$$

We can naturally identify $H^{0,1}\left(\mathbb{R} \times \mathbb{T}^{2}\right)$ and $h^{1} L^{2}$ by via the Fourier transform in the periodic variable $y$ as follows: for $\psi \in H^{0,1}\left(\mathbb{R} \times \mathbb{T}^{2}\right)$ we define the vector $\vec{\psi}=\left\{\psi_{p}\right\}_{p}$ where

$$
\psi_{p}(x):=\frac{1}{(2 \pi)^{2}} \int_{\mathbb{T}^{2}} \psi(x, y) e^{-i\langle p, y\rangle} d y
$$

is the sequence of periodic Fourier coefficients of $\psi$. Conversely, for any $\vec{v} \in h^{1} L^{2}$, we define

$$
\underline{\vec{v}}(x, y):=\sum_{p \in \mathbb{Z}^{2}} v_{p}(x) e^{i\langle y, p\rangle} .
$$

Clearly the two operations invert one another and are isometries 8 .

\footnotetext{
${ }^{8} \mathrm{Up}$ to the normalizing constants in the definition of the Fourier transform.
} 
2.2. Function spaces. For $C=\left[-\frac{1}{2}, \frac{1}{2}\right)^{3} \subset \mathbb{R}^{3}$ and $z \in \mathbb{R}^{3}$, we denote by $C_{z}=z+C$ the translate of $C$ by $z$ and define the sharp projection operator $P_{C_{z}}$ as follows:

$$
\mathcal{F}\left(P_{C_{z}} f\right)=\chi_{C_{z}}(\xi) \mathcal{F}(f)(\xi)
$$

We use the same modifications of the atomic and variation space norms that were employed in [31, 32, 34. Namely, for $s \in \mathbb{R}$, we define:

$$
\|u\|_{X_{0}^{s}(\mathbb{R})}^{2}=\sum_{z \in \mathbb{Z}^{3}}\langle z\rangle^{2 s}\left\|P_{C_{z}} u\right\|_{U_{\Delta}^{2}\left(\mathbb{R} ; L^{2}\right)}^{2}, \quad\|u\|_{Y^{s}(\mathbb{R})}^{2}=\sum_{z \in \mathbb{Z}^{3}}\langle z\rangle^{2 s}\left\|P_{C_{z}} u\right\|_{V_{\Delta}^{2}\left(\mathbb{R} ; L^{2}\right)}^{2} .
$$

We refer to [28, 31, 32] for the description and properties of the spaces $U_{\Delta}^{p}$ and $V_{\Delta}^{p}$. The norms $X_{0}^{s}$ and $Y^{s}$ are both stronger than the $L^{\infty}\left(\mathbb{R} ; H^{s}\right)$ norm and weaker than the $U_{\Delta}^{2}\left(\mathbb{R}: H^{s}\right)$ norm. In fact, they satisfy the following nesting property:

$$
U_{\Delta}^{2}\left(\mathbb{R} ; H^{s}\right) \hookrightarrow X_{0}^{s}(\mathbb{R}) \hookrightarrow Y^{s}(\mathbb{R}) \hookrightarrow V_{\Delta}^{2}\left(\mathbb{R} ; H^{s}\right) \hookrightarrow U_{\Delta}^{p}\left(\mathbb{R} ; H^{s}\right) \hookrightarrow L^{\infty}\left(\mathbb{R} ; H^{s}\right)
$$

for any $p>2$. For a compact interval $I \subset \mathbb{R}$, we also need the restriction norms $X^{s}(I)$ and $Y^{s}(I)$ defined in the usual way:

$$
\|u\|_{X^{s}(I)}=\inf \left\{\|v\|_{X_{0}^{s}(\mathbb{R})}: v \in X_{0}^{s}(\mathbb{R}) \text { satisfying } v_{\mid I}=u_{\mid I}\right\}
$$

and similarly for $Y^{s}(I)$. Our main solution space is then

$X_{c}^{s}(\mathbb{R}):=\left\{u \in C\left(\mathbb{R}: H^{s}\left(\mathbb{R} \times \mathbb{T}^{2}\right)\right): \phi_{-\infty}:=\lim _{t \rightarrow-\infty} e^{-i t \Delta} u(t)\right.$ exists in $H^{s}$, and $\left.u(t)-e^{i t \Delta} \phi_{-\infty} \in X_{0}^{s}(\mathbb{R})\right\}$ equipped with the norm:

$$
\|u\|_{X^{s}(\mathbb{R})}^{2}:=\|\phi\|_{H^{s}\left(\mathbb{R} \times \mathbb{T}^{2}\right)}^{2}+\left\|u-e^{i t \Delta} \phi_{-\infty}\right\|_{X_{0}^{s}(\mathbb{R})}^{2} \simeq \sup _{K \subset \mathbb{R}, K \text { compact }}\|u\|_{X^{s}(K)}^{2}
$$

and we can extend the second definition of the $X^{s}$-norm to arbitrary subintervals of $\mathbb{R}$. We also consider $X_{c, \text { loc }}^{1}(I)$ to be the set of all functions in $C_{\text {loc }}\left(I: H^{1}\right)$ whose $X^{1}(J)$-norm is finite for every compact subset $J \subset I$.

To control the nonlinearity on an interval $I=(a, b)$ we use the norm:

$$
\|h\|_{N^{s}(I)}:=\left\|\int_{a}^{t} e^{i(t-s) \Delta} h(s) d s\right\|_{X^{s}(I)} .
$$

We mostly use this norm for $s=1$ and in this case, we omit the $s$ in the notation.

In addition to the above norms, we need the following time-divisible norm:

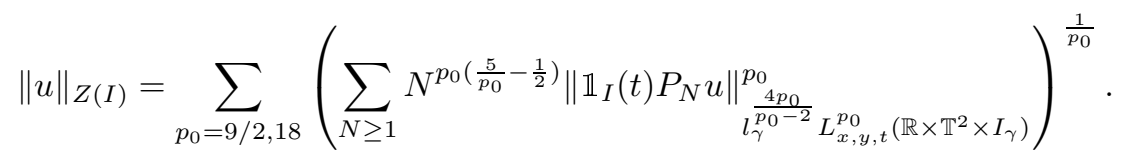

where $I_{\gamma}=[2 \pi \gamma, 2 \pi(\gamma+1)]$. $Z$ is a weaker norm than $X^{1}$. In fact, from Lemma 4.1 below,

$$
\|u\|_{Z(I)} \lesssim\|u\|_{X^{1}(I)}
$$

We will also need the following proposition which is the analogue of Poposition 2.11 of [31] or Proposition 2.10 of [32]:

Proposition $2.1(\underline{31}, 32])$. If $f \in L_{t}^{1}\left(I, H^{1}\left(\mathbb{R} \times \mathbb{T}^{2}\right)\right)$, then

$$
\|f\|_{N(I)} \lesssim \sup _{\substack{v \in Y^{-1}(I) \\\|v\|_{Y^{-1}(I)} \leq 1}} \int_{I \times\left(\mathbb{R} \times \mathbb{T}^{2}\right)} f(x, t) \overline{v(x, t)} d x d t
$$


In particular, the following estimate holds for any smooth function $g$ on an interval $I=[a, b]$ :

$$
\|g\|_{X^{1}(I)} \lesssim\|g(0)\|_{H^{1}\left(\mathbb{R} \times \mathbb{T}^{2}\right)}+\left(\sum_{N}\left\|P_{N}\left(i \partial_{t}+\Delta\right) g\right\|_{L_{t}^{1}\left(I, H_{x}^{1}\left(\mathbb{R} \times \mathbb{T}^{2}\right)\right)}^{2}\right)^{1 / 2} .
$$

\section{Global Strichartz estimates}

We recall the Strichartz estimates from $[8]$ : for any $p>4$, there holds that

$$
\left\|e^{i t \Delta_{\mathbb{T}^{3}}} P_{N} f\right\|_{L_{x, t}^{p}\left(\mathbb{T}_{x}^{3} \times \mathbb{T}_{t}\right)} \lesssim N^{\left(\frac{3}{2}-\frac{5}{p}\right)}\|f\|_{L^{2}\left(\mathbb{T}^{3}\right)}
$$

This bound can of course be adapted to the case of $\mathbb{R} \times \mathbb{T}^{2}$. However, in order to prove scattering estimates, we need to obtain global in time bounds. This is only possible by using the $\mathbb{R}$ component of our manifold. But this only supports linear solutions that decay much slower than on $\mathbb{R}^{3}$. As a result, we need to distinguish between the "local in time" integrability, similar to the one in $\mathbb{R}^{3}$ (or $\mathbb{T}^{3}$ ) and the "global in time" integrability, similar to the one on $\mathbb{R}$. This is done by decomposing

$$
\mathbb{R}_{t}=\bigcup_{\gamma \in \mathbb{Z}} 2 \pi[\gamma, \gamma+1) .
$$

In this section, we prove the following result:

Theorem 3.1. Let $N \geq 1$ be dyadic, then

$$
\left\|e^{i t \Delta_{\mathbb{R} \times \mathbb{T}^{2}}} P_{\leq N} u_{0}\right\|_{l_{\gamma}^{q} L_{x, y, t}^{p}\left(\mathbb{R} \times \mathbb{T}^{2} \times[2 \pi \gamma, 2 \pi(\gamma+1)]\right)} \lesssim N^{\left(\frac{3}{2}-\frac{5}{p}\right)}\left\|u_{0}\right\|_{L^{2}\left(\mathbb{R} \times \mathbb{T}^{2}\right)} .
$$

whenever

$$
p>4 \quad \text { and } \quad \frac{2}{q}+\frac{1}{p}=\frac{1}{2} .
$$

In the estimate above, we see that the loss in derivatives is dictated by the local $(3 d)$ geometry, while the global integrability condition is dictated by the global $(1 d)$ geometry. Theorem 3.1 follows directly by duality from the following more precise estimate

Lemma 3.2. For any $h \in C_{c}^{\infty}\left(\mathbb{R}_{x} \times \mathbb{T}_{y}^{2} \times \mathbb{R}_{t}\right)$, there holds that

$$
\begin{aligned}
& \left\|\int_{s \in \mathbb{R}} e^{-i s \Delta_{\mathbb{R} \times \mathbb{T}^{2}}} P_{\leq N} h(x, y, s) d s\right\|_{L_{x, y}^{2}\left(\mathbb{R} \times \mathbb{T}^{2}\right)} \\
& \lesssim N^{\left(\frac{3}{2}-\frac{5}{p}\right)}\|h\|_{l_{\gamma}^{2} L_{x, y, t}^{p^{\prime}}\left(\mathbb{R} \times \mathbb{T}^{2} \times[2 \pi \gamma, 2 \pi(\gamma+1)]\right)}+N^{\left(1-\frac{3}{p}\right)}\|h\|_{l_{\gamma}^{q^{\prime}} L_{x, y, t}^{p^{\prime}}\left(\mathbb{R} \times \mathbb{T}^{2} \times[2 \pi \gamma, 2 \pi(\gamma+1)]\right)}
\end{aligned}
$$

for any $(q, p)$ satisfying (3.3).

Proof of Lemma 3.1. In order to distinguish between the large and small time scales, we choose a smooth partition of unity

$$
1=\sum_{\gamma \in \mathbb{Z}} \chi(t-2 \pi \gamma)
$$


with $\chi$ supported in $[-2 \pi, 2 \pi]$. We also denote by $h_{\alpha}(t):=\chi(t) h(2 \pi \alpha+t)$. Using the semigroup property and the unitarity of $e^{i t \Delta_{\mathbb{R} \times \mathbb{T}^{2}}}$, we compute directly that

$$
\begin{aligned}
& \left\|\int_{s \in \mathbb{R}} e^{-i s \Delta_{\mathbb{R} \times \mathbb{T}^{2}}} P_{\leq N} h(x, y, s) d s\right\|_{L_{x, y}^{2}\left(\mathbb{R} \times \mathbb{T}^{2}\right)}^{2} \\
& =\iint_{s, t \in \mathbb{R}}\left\langle e^{-i s \Delta_{\mathbb{R} \times \mathbb{T}^{2}}} P_{\leq N} h(s), e^{-i t \Delta_{\mathbb{R} \times \mathbb{T}^{2}}} P_{\leq N} h(t)\right\rangle_{L_{x, y}^{2} \times L_{x, y}^{2}} d s d t \\
& =\sum_{\alpha, \beta} \iint_{s, t \in \mathbb{R}}\left\langle\chi(s-2 \pi \alpha) e^{-i s \Delta_{\mathbb{R} \times \mathbb{T}^{2}}} P_{\leq N} h(s), \chi(t-2 \pi \beta) e^{-i t \Delta_{\mathbb{R} \times \mathbb{T}^{2}}} P_{\leq N} h(t)\right\rangle_{L_{x, y}^{2} \times L_{x, y}^{2}} d s d t \\
& =\sum_{\alpha, \beta} \iint_{s, t \in[-2 \pi, 2 \pi]}\left\langle e^{-i(2 \pi(\alpha-\beta)+s) \Delta_{\mathbb{R} \times \mathbb{T}^{2}}} P_{\leq N} h_{\alpha}(s), e^{-i t \Delta_{\mathbb{R} \times \mathbb{T}^{2}}} P_{\leq N} h_{\beta}(t)\right\rangle_{L_{x, y}^{2} \times L_{x, y}^{2}} d s d t \\
& =\sigma_{\text {diag }}+\sigma_{\text {non-diag }}
\end{aligned}
$$

where

$$
\begin{aligned}
\sigma_{\text {diag }} & =\sum_{|\alpha-\beta| \leq 9} \iint_{s, t}\left\langle e^{-i(2 \pi(\alpha-\beta)+s) \Delta_{\mathbb{R} \times \mathbb{T}^{2}}} P_{\leq N} h_{\alpha}(s), e^{-i t \Delta_{\mathbb{R} \times \mathbb{T}^{2}}} P_{\leq N} h_{\beta}(t)\right\rangle_{L_{x, y}^{2} \times L_{x, y}^{2}} d s d t \\
\sigma_{\text {non-diag }} & =\sum_{\alpha, \gamma \in \mathbb{Z},|\gamma| \geq 10} \iint_{s, t}\left\langle e^{-i(s-2 \pi \gamma) \Delta_{\mathbb{R} \times \mathbb{T}^{2}}} P_{\leq N} h_{\alpha}(s), e^{-i t \Delta_{\mathbb{R} \times \mathbb{T}^{2}}} P_{\leq N} h_{\alpha+\gamma}(t)\right\rangle_{L_{x, y}^{2} \times L_{x, y}^{2}} d s d t .
\end{aligned}
$$

The diagonal sum dictates the loss of derivative, while the non-diagonal sum dictates the decay.

Diagonal Estimates. Here, we use the following result from [8] for any $f \in L^{2}\left(\mathbb{T}^{2}\right)$ and any $p>4$, there holds that

$$
\left\|e^{i t \Delta_{\mathbb{T}^{2}}} P_{\leq N}^{y} f\right\|_{L^{p}\left(\mathbb{T}_{y}^{2} \times \mathbb{T}_{t}\right)} \lesssim N^{\left(1-\frac{4}{p}\right)}\|f\|_{L^{2}\left(\mathbb{T}_{y}^{2}\right)} .
$$

This implies that for $p>4$,

$$
\left\|e^{i t \Delta_{\mathbb{R} \times \mathbb{T}^{2}}} P_{\leq N} f\right\|_{L_{x, y, t}^{p}\left(\mathbb{R} \times \mathbb{T}^{2} \times[0,2 \pi]\right)} \lesssim N^{\left(\frac{3}{2}-\frac{5}{p}\right)}\|f\|_{L_{x, y}^{2}\left(\mathbb{R} \times \mathbb{T}^{2}\right)} .
$$

Indeed, using the Hausdorff-Young, Hölder, Minkowski and Plancherel inequalities, we have that

$$
\begin{aligned}
& \left\|e^{i t \Delta_{\mathbb{R} \times \mathbb{T}^{2}}} P_{\leq N} f\right\|_{L_{x, y, t}^{p}\left(\mathbb{R} \times \mathbb{T}^{2} \times[-2 \pi, 2 \pi]\right)}=\left\|e^{i t \Delta_{\mathbb{R} \times \mathbb{T}^{2}}} P_{\leq N} f\right\|_{L_{x}^{p}\left(\mathbb{R} ; L_{y, t}^{p}\left(\mathbb{T}^{2} \times[-2 \pi, 2 \pi]\right)\right)} \\
& \lesssim N^{\left(\frac{1}{2}-\frac{1}{p}\right)}\left\|e^{-i t|\xi|^{2}} \mathcal{F}_{\xi} e^{i t \Delta_{\mathbb{T}^{2}}} P_{\leq N} f\right\|_{L_{\xi}^{2}\left(\mathbb{R} ; L_{y, t}^{p}\left(\mathbb{T}^{2} \times[-2 \pi, 2 \pi]\right)\right)} \\
& \lesssim N^{\left(\frac{1}{2}-\frac{1}{p}\right)}\left\|e^{i t \Delta_{\mathbb{T}^{2}}} P_{\leq N}^{y} \mathcal{F}_{\xi} f\right\|_{L_{\xi}^{2}\left(\mathbb{R} ; L_{y, t}^{p}\left(\mathbb{T}^{2} \times[-2 \pi, 2 \pi]\right)\right)} \\
& \lesssim N^{\left(\frac{3}{2}-\frac{5}{p}\right)}\left\|\mathcal{F}_{\xi} f\right\|_{L_{\xi}^{2} L_{y}^{2}}=N^{\left(\frac{3}{2}-\frac{5}{p}\right)}\|f\|_{L_{x, y}^{2}\left(\mathbb{R} \times \mathbb{T}^{2}\right)} \text {. }
\end{aligned}
$$

Again, by duality, this implies that

$$
\left\|\int_{s \in \mathbb{R}} e^{-i s \Delta_{\mathbb{R} \times \mathbb{T}^{2}}} P_{\leq N} h(s) d s\right\|_{L_{x, y}^{2}\left(\mathbb{R} \times \mathbb{T}^{2}\right)} \lesssim N^{\left(\frac{3}{2}-\frac{5}{p}\right)}\|h\|_{L_{x, y, t}^{p^{\prime}}\left(\mathbb{R} \times \mathbb{T}^{2} \times[-2 \pi, 2 \pi]\right)}
$$


whenever $h$ is supported in $[-2 \pi, 2 \pi]$. And consequently,

$$
\begin{aligned}
\sigma_{\text {diag }} & =\sum_{\alpha \in \mathbb{Z},|\gamma| \leq 9} \iint_{s, t \in \mathbb{R}}\left\langle e^{-i(s-2 \pi \gamma) \Delta_{\mathbb{R} \times \mathbb{T}^{2}}} P_{\leq N} h_{\alpha}(s), e^{-i t \Delta_{\mathbb{R} \times \mathbb{T}^{2}}} P_{\leq N} h_{\alpha+\gamma}(t)\right\rangle_{L_{x, y}^{2} \times L_{x, y}^{2}} d s d t \\
& \leq \sum_{\alpha,|\gamma| \leq 9}\left\|\int_{s \in \mathbb{R}} e^{-i s \Delta_{\mathbb{R} \times \mathbb{T}^{2}} P_{\leq N} h_{\alpha}(2 \pi \gamma+s) d s}\right\|_{L_{x, y}^{2}\left(\mathbb{R} \times \mathbb{T}^{2}\right)} \| \int_{s \in \mathbb{R}} e^{-i s \Delta_{\mathbb{R} \times \mathbb{T}^{2}} P_{\leq N} h_{\alpha+\gamma}(s) d s \|_{L_{x, y}^{2}\left(\mathbb{R} \times \mathbb{T}^{2}\right)}} \\
& \lesssim N^{2\left(\frac{3}{2}-\frac{5}{p}\right)} \sum_{\alpha}\left\|h_{\alpha}\right\|_{L_{x, y, t}^{p^{\prime}}}^{2}\left(\mathbb{R} \times \mathbb{T}^{2} \times[-2 \pi, 2 \pi]\right)
\end{aligned}
$$

This is controlled by the first term in the righthand side of (3.4).

Nondiagonal Estimates. We can estimate the non diagonal sum as follows using (3.8) proved below:

$$
\begin{aligned}
\sigma_{\text {non-diag }} & =\sum_{\alpha, \gamma \in \mathbb{Z},|\gamma| \geq 10} \int_{t}\left\langle\int_{s} e^{-i(s-t-2 \pi \gamma) \Delta_{\mathbb{R} \times \mathbb{T}^{2}}} P_{\leq N} h_{\alpha}(s) d s, P_{\leq N} h_{\alpha+\gamma}(t)\right\rangle_{L_{x, y}^{2} \times L_{x, y}^{2}} d t \\
& \lesssim N^{\left(2-\frac{6}{p}\right)} \sum_{\alpha, \gamma \in \mathbb{Z},|\gamma| \geq 3}|\gamma|^{\frac{1}{p}-\frac{1}{2}}\left\|h_{\alpha}\right\|_{L_{x, y, t}^{p^{\prime}}}\left\|h_{\alpha+\gamma}\right\|_{L_{x, y, t}^{p^{\prime}}} \\
& \lesssim N^{\left(2-\frac{6}{p}\right)}\left\|\left\{h_{\alpha}\right\}_{\alpha}\right\|_{l_{\alpha}^{q^{\prime}} L_{x, y, t}^{p^{\prime}}\left(\mathbb{R} \times \mathbb{T}^{2} \times[-2 \pi, 2 \pi]\right)}^{2}, \quad\left(\frac{2}{q}=\frac{1}{2}-\frac{1}{p}\right)
\end{aligned}
$$

where we have used the discrete Hardy-Littlewood-Sobolev inequality in the last step. Together with (3.6), this finishes the proof of (3.4) once we prove Lemma 3.3 .

Lemma 3.3. Suppose that $\gamma \in \mathbb{Z}$ satisfies $|\gamma| \geq 3$ and that $p>4$. For any $h \in L_{x, y, s}^{p^{\prime}}\left(\mathbb{R} \times \mathbb{T}^{2} \times[-2 \pi, 2 \pi]\right)$, there holds that:

$$
\left\|\int_{s \in \mathbb{R}} \chi(s) e^{i(t-s+2 \pi \gamma) \Delta_{\mathbb{R} \times \mathbb{T}^{2}}} P_{\leq N} h(s) d s\right\|_{L_{x, y, t}^{p}\left(\mathbb{R} \times \mathbb{T}^{2} \times[-2 \pi, 2 \pi]\right)} \lesssim|\gamma|^{\frac{1}{p}-\frac{1}{2}} N^{\left(2-\frac{6}{p}\right)}\|h\|_{L_{x, y, s}^{p^{\prime}}\left(\mathbb{R} \times \mathbb{T}^{2} \times[-2 \pi, 2 \pi]\right)} .
$$

Proof. Without loss of generality, we may assume that:

$$
h=\chi(s) P_{\leq N} h, \quad \text { and }\|h\|_{L_{x, y, t}^{p^{\prime}}\left(\mathbb{R} \times \mathbb{T}^{2} \times[-2 \pi, 2 \pi]\right)}=1
$$

and we define

We also define the Kernel

$$
g(x, y, t)=\int_{s \in \mathbb{R}} e^{i(t-s+2 \pi \gamma) \Delta_{\mathbb{R} \times \mathbb{T}^{2}} h(x, y, s) d s .}
$$

$$
\begin{aligned}
K_{N}(x, y, t) & =\sum_{k \in \mathbb{Z}^{2}} \int_{\mathbb{R}_{\xi}}\left[\eta_{\leq N}^{1}(\xi)\right]^{2}\left[\eta_{\leq N}^{1}\left(k_{1}\right) \eta_{\leq N}^{1}\left(k_{2}\right)\right]^{2} e^{i\left[x \cdot \xi+y \cdot k+t\left(|k|^{2}+|\xi|^{2}\right)\right]} d \xi \\
& =\left[\int_{\mathbb{R}_{\xi}}\left[\eta_{\leq N}^{1}(\xi)\right]^{2} e^{i\left(x \cdot \xi+t|\xi|^{2}\right)} d \xi\right] \cdot\left[\sum_{k \in \mathbb{Z}^{2}}\left[\eta_{\leq N}^{1}\left(k_{1}\right) \eta_{\leq N}^{1}\left(k_{2}\right)\right]^{2} e^{i\left(y \cdot k+t|k|^{2}\right)}\right] \\
& =K_{N}^{\mathbb{R}}(x, t) \otimes K_{N}^{\mathbb{T}^{2}}(y, t) .
\end{aligned}
$$

and introduce $K_{N, \gamma}(x, y, t):=K_{N}(x, y, 2 \pi \gamma+t)$, so that $g(x, y, t)=K_{N, \gamma} *_{x, y, t} h$. We have the following straightforward bounds:

$$
\begin{aligned}
\left\|K_{N, \gamma}\right\|_{L_{x, y, t}^{\infty}} & \lesssim|\gamma|^{-\frac{1}{2}} N^{2} \\
\left\|\mathcal{F}_{x, y, t} K_{N, \gamma}\right\|_{L_{\xi, k, \tau}^{\infty}} & \lesssim 1
\end{aligned}
$$


The first estimates follows from stationary phase estimates in $x$ and direct counting in $k$ (see also (3.16)). The second estimate is direct.

For $\alpha$ a dyadic number, we define $g^{\alpha}(x, y, t)=\alpha^{-1} g(x, y, t) \mathbb{1}_{\{\alpha / 2 \leq|g|<\alpha\}}$ which has modulus in $[1 / 2,1]$. We define $h^{\beta}$ similarly for $\beta \in 2^{\mathbb{Z}}$. Clearly, $g=\sum_{\alpha} \alpha g^{\alpha}$. Besides,

$$
\begin{aligned}
\|g\|_{L_{x, y, t}^{p}}^{p} & =\left\langle|g|^{p-2} g, g\right\rangle \\
& =\sum_{\alpha, \beta} \alpha^{p-1} \beta\left\langle\left|g^{\alpha}\right|^{p-2} g^{\alpha}, K_{N, \gamma} * h^{\beta}\right\rangle \\
& =\left[\sum_{\mathcal{S}_{1}}+\sum_{\mathcal{S}_{2}}+\sum_{\mathcal{S}_{3}}\right] \alpha^{p-1} \beta\left\langle\left|g^{\alpha}\right|^{p-2} g^{\alpha}, K_{N, \gamma} * h^{\beta}\right\rangle=\Sigma_{1}+\Sigma_{2}+\Sigma_{3},
\end{aligned}
$$

where

$$
\begin{aligned}
& \mathcal{S}_{1}=\left\{(\alpha, \beta): C|\gamma|^{-1 / 2} N^{2} \leq \alpha \beta^{p^{\prime}-1}\right\}, \\
& \mathcal{S}_{2}=\left\{(\alpha, \beta): \alpha \beta^{p^{\prime}-1} \leq C N|\gamma|^{-1 / 2}\right\}, \\
& \mathcal{S}_{3}=\left\{(\alpha, \beta): C N|\gamma|^{-1 / 2} \leq \alpha \beta^{p^{\prime}-1} \leq C|\gamma|^{-1 / 2} N^{2}\right\}
\end{aligned}
$$

for $C$ a large constant to be fixed later. In each of the three cases defined above and for any fixed $\alpha, \beta$, we will decompose $K_{N, \gamma}=K_{N, \gamma ; \alpha, \beta}^{1}+K_{N, \gamma ; \alpha, \beta}^{2}$ and estimate the corresponding contributions as follows:

$$
\begin{aligned}
\left\langle\left|g^{\alpha}\right|^{p-2} g^{\alpha}, K_{N, \gamma ; \alpha, \beta}^{1} * h^{\beta}\right\rangle & \lesssim\left\|K_{N, \gamma ; \alpha, \beta}^{1}\right\|_{L_{x, y, t}^{\infty}}\left\|g^{\alpha}\right\|_{L^{1}}\left\|h^{\beta}\right\|_{L^{1}}, \\
\left\langle\left|g^{\alpha}\right|^{p-2} g^{\alpha}, K_{N, \gamma ; \alpha, \beta}^{2} * h^{\beta}\right\rangle & \lesssim\left\|\mathcal{F}_{x, y, t} K_{N, \gamma ; \alpha, \beta}^{2}\right\|_{L_{\xi, k, \tau}^{\infty}}\left\|g^{\alpha}\right\|_{L^{2}}\left\|h^{\beta}\right\|_{L^{2}} .
\end{aligned}
$$

In what follows, we denote by $S_{\alpha}:=\operatorname{supp}\left(g_{\alpha}\right)$ and $E_{\beta}:=\operatorname{supp}\left(h_{\beta}\right)$. Then, by assumption

$$
\|g\|_{L_{x, y, t}^{p}}^{p} \simeq \sum_{\alpha} \alpha^{p}\left|S_{\alpha}\right|, \quad \sum_{\beta} \beta^{\frac{p}{p-1}}\left|E_{\beta}\right| \simeq 1, \quad\left\|g^{\alpha}\right\|_{L_{x, y, t}^{p}} \simeq\left|S_{\alpha}\right|^{\frac{1}{p}} .
$$

We will bound $\Sigma_{1}, \Sigma_{2}, \Sigma_{3}$ in (3.12) separately:

$\Sigma_{1}$, the large levels: $C|\gamma|^{-1 / 2} N^{2} \leq \alpha \beta^{p^{\prime}-1}$. In this case, we use the decomposition (3.13) with $K^{2}=0$ and the bound (3.11). This gives

$$
\Sigma_{1} \leq \sum_{\alpha, \beta ; C|\gamma|^{-1 / 2} N^{2} \leq \alpha \beta^{p^{\prime}-1}} \alpha^{p-1} \beta\left\|K_{N, \gamma}\right\|_{L_{x, y, t}^{\infty}}\left|S_{\alpha}\right|\left|E_{\beta}\right| \lesssim C^{-1} \sum_{\alpha, \beta} \alpha^{p}\left|S_{\alpha}\right| \beta^{p^{\prime}}\left|E_{\beta}\right| \lesssim C^{-1}\|g\|_{L_{x, y, t}^{p}}^{p}
$$

$\Sigma_{2}$, the small levels: $\alpha \beta^{p^{\prime}-1} \leq C N|\gamma|^{-1 / 2}$. In this case, we use the decomposition (3.13) with $K^{1}=0$ and (3.11). This gives

$$
\Sigma_{2} \leq \sum_{(\alpha, \beta) \in \mathcal{S}_{2}} \alpha^{p-1} \beta\left|S_{\alpha}\right|^{\frac{1}{2}}\left|E_{\beta}\right|^{\frac{1}{2}} \leq \sum_{\alpha, \beta ; \alpha \beta^{p^{\prime}-1} \leq C N|\gamma|^{-1 / 2}}\left[\alpha^{p}\left|S_{\alpha}\right|\right]^{\frac{1}{2}}\left[\beta^{p^{\prime}}\left|E_{\beta}\right|\right]^{\frac{1}{2}}\left[\alpha \beta^{p^{\prime}-1}\right]^{\frac{p-2}{2}} .
$$

Using Schur's test, we can sum in $\alpha, \beta$ and this gives

$$
\Sigma_{2} \lesssim C^{\frac{p-2}{2}}\|g\|_{L_{x, y, t}^{p}}^{\frac{p}{2}} N^{\frac{p-2}{2}}|\gamma|^{-\frac{p-2}{4}} .
$$

\footnotetext{
${ }^{9}$ This is not be confused with $h_{\alpha}$ defined previous to Lemma 3.3
} 
$\Sigma_{3}$, the medium levels: $C N|\gamma|^{-1 / 2} \leq \alpha \beta^{p^{\prime}-1} \leq C|\gamma|^{-1 / 2} N^{2}$. In this case, we will decompose the kernel into two components as in (3.13) which satisfy

$$
\begin{aligned}
\left\|K_{N, \gamma ; \alpha, \beta}^{1}\right\|_{L_{x, y, t}^{\infty}} & \lesssim C^{-1} \alpha \beta^{p^{\prime}-1} \\
\left\|\mathcal{F}_{t, x, y} K_{N, \gamma ; \alpha, \beta}^{2}\right\|_{L_{\xi, k, \tau}^{\infty}} & \lesssim \varepsilon N^{\varepsilon} C\left(\alpha \beta^{p^{\prime}-1}\right)^{-1}|\gamma|^{-1 / 2} .
\end{aligned}
$$

Assuming this, we finish the proof as follows:

$$
\begin{aligned}
\Sigma_{3} & \lesssim C^{-1} \sum_{\mathcal{S}_{3}} \alpha^{p} \beta^{p^{\prime}}\left|S_{\alpha}\right|\left|E_{\beta}\right|+N^{\varepsilon} C \sum_{\mathcal{S}_{3}} \alpha^{p-2} \beta^{2-p^{\prime}}\left|S_{\alpha}\right|^{\frac{1}{2}}\left|E_{\beta}\right|^{\frac{1}{2}}|\gamma|^{-1 / 2} \\
& \lesssim C^{-1}\|g\|_{L_{x, y, t}^{p}}^{p}+N^{\varepsilon} C \sum_{\mathcal{S}_{3}}\left[\alpha^{p}\left|S_{\alpha}\right|\right]^{\frac{1}{2}}\left[\beta^{p^{\prime}}\left|E_{\beta}\right|\right]^{\frac{1}{2}}\left[\alpha \beta^{p^{\prime}-1}\right]^{\frac{p-4}{2}}|\gamma|^{-1 / 2} \\
& \lesssim C^{-1}\|g\|_{L_{x, y, t}^{p}}^{p}+\|g\|_{L_{x, y, t}^{p}}^{\frac{p}{2}} C^{\frac{p-2}{2}} N^{(p-4+\varepsilon)}|\gamma|^{-\frac{p-2}{4}} .
\end{aligned}
$$

As a result we finally obtain

$$
\|g\|_{L_{x, y, t}^{p}}^{p}=\Sigma_{1}+\Sigma_{2}+\Sigma_{3} \lesssim C^{-1}\|g\|_{L_{x, y, t}^{p}}^{p}+C^{\frac{p-2}{2}}\|g\|_{L_{x, y, t}^{p}}^{\frac{p}{2}}\left[N^{\frac{p-2}{2}}|\gamma|^{-\frac{p-2}{4}}+N^{p-4+\varepsilon}|\gamma|^{-\frac{p-2}{4}}\right] .
$$

We now fix the universal constant $C$ so that it is large enough to absorb the first term on the RHS in the LHS and we get:

$$
\|g\|_{L_{x, y, t}^{p}}^{p} \lesssim C\|g\|_{L_{x, y, t}^{p}}^{\frac{p}{2}}|\gamma|^{-\frac{p-2}{4}} \max \left(N^{(p-4+\varepsilon)}, N^{\frac{p-2}{2}}\right) \lesssim\|g\|_{L_{x, y, t}^{p}}^{\frac{p}{2}}|\gamma|^{-\frac{p-2}{4}} N^{p-3}
$$

if $p>4$. This finishes the proof of (3.8) once we justify the decomposition (3.14).

In order to get the decomposition (3.14), we need a minor/major arc decomposition of the Kernel $K_{N}^{\mathbb{T}^{2}}$. We recall the following bound from [8, Lemma 3.18]: assume that $1 \leq a \leq q \leq N,(a, q)=1$ and $|t / 2 \pi-a / q| \leq 1 / N q$, then

$$
\left\|K_{N}^{\mathbb{T}^{2}}(\cdot, t)\right\|_{L_{y}^{\infty}\left(\mathbb{T}^{2}\right)} \lesssim \frac{N^{2}}{q\left(1+N^{2}|t / 2 \pi-a / q|\right)} .
$$

From (3.10) and the explicit formula in $\mathbb{R}$, we directly get that, under the above conditions, the first estimate in (3.11) can be further refined into:

$$
\left\|K_{N, \gamma}(t)\right\|_{L_{x, y}^{\infty}\left(\mathbb{R} \times \mathbb{T}^{2}\right)} \lesssim|\gamma|^{-1 / 2} \frac{N^{2}}{q\left(1+N^{2}|t / 2 \pi-a / q|\right)} .
$$

For $L \in 2^{\mathbb{Z}}$, we will need the following fine-scale time-cutoff functions $\phi_{\geq L}(\cdot):=\eta^{1}(L \cdot)$ and $\phi_{L}=$ $\phi_{\geq L}-\phi_{\geq 2 L}$. Let $n$ be such that $N=2^{n}$ and for $0 \leq k \leq n-10, j+k \leq n$, define the time projectors

$$
\begin{aligned}
p_{k, j}(s) & :=\sum_{2^{k} \leq q \leq 2^{k+1}} \sum_{(a, q)=1,1 \leq a \leq q} \phi_{N 2^{k+j+10}}(s / 2 \pi-a / q) \quad \text { for } j \leq n-k-1, \\
p_{k, n-k}(s) & :=\sum_{2^{k} \leq q \leq 2^{k+1}} \sum_{(a, q)=1,1 \leq a \leq q} \phi_{\geq 2^{10} N^{2}}(s / 2 \pi-a / q)
\end{aligned}
$$

and let $e$ be such that

$$
1=\sum_{k=0}^{n-10} \sum_{j ; j+k \leq n} p_{k, j}(t)+e(t) .
$$

We now define

$$
K_{N, \gamma ; \alpha, \beta}^{1}(x, y, t):=\left[e(t)+\sum_{\mathcal{S}_{M}} p_{k, j}(t)+\sum_{\mathcal{S}_{m}} \rho_{j, k} p_{k, 0}(t)\right] K_{N, \gamma}(x, y, t) .
$$


where we used the following notations:

$$
\begin{gathered}
\rho_{k, j}:=2^{-j} \text { if } j<n-k \text { and } \rho_{k, n-k}:=2^{-n+k+1} \\
\mathcal{S}_{M}:=\left\{(j, k): 0 \leq k \leq n-10,0 \leq j+k \leq n ; 2^{n+j} \leq C^{-1} \alpha \beta^{p^{\prime}-1}|\gamma|^{1 / 2}\right\} \\
\mathcal{S}_{m}:=\left\{(j, k): 0 \leq k \leq n-10,0 \leq j+k \leq n ; 2^{n+j} \geq C^{-1} \alpha \beta^{p^{\prime}-1}|\gamma|^{1 / 2}\right\}
\end{gathered}
$$

the defining sets of the major and minor arcs. The $\rho_{j, k}$ are introduced to cancel the large zero frequency mode of $K_{n, \gamma ; \alpha, \beta}^{2}(x, y, t)$ (cf. (3.20) and (3.23) $)$.

By direct inspection, we see that the major arcs are disjoint:

$$
\phi_{N 2^{k+j+10}}(s / 2 \pi-a / q) \phi_{N 2^{k^{\prime}+j^{\prime}+10}}\left(s / 2 \pi-a^{\prime} / q^{\prime}\right) \neq 0 \Rightarrow k=k^{\prime},(a, q)=\left(a^{\prime}, q^{\prime}\right),\left|j-j^{\prime}\right| \leq 3 .
$$

In particular,

$$
0 \leq p_{k, j} \leq 1 \quad \text { and } \quad p_{k, j}(t) p_{k^{\prime}, j^{\prime}}(t) \neq 0 \Rightarrow k=k^{\prime} ;\left|j-j^{\prime}\right| \leq 3 .
$$

If $e(t) \neq 0$, then either $|t / 2 \pi-a / q| \geq 2^{-n} / q$ or $q \gtrsim 2^{n}$. In both cases, from (3.16), we see that

$$
\left\|e(t) K_{N, \gamma}\right\|_{L_{x, y, t}^{\infty}} \lesssim 2^{n}|\gamma|^{-1 / 2} .
$$

Invoking (3.16) again, we deduce that

$$
\left\|K_{N, \gamma}(x, t) \sum_{\mathcal{S}_{M}} p_{k, j}(t)\right\|_{L_{x, y, t}^{\infty}} \lesssim \sup _{\mathcal{S}_{M}}\left\|p_{k, j} K_{N, \gamma}\right\|_{L_{x, y, t}^{\infty}} \lesssim C^{-1} \alpha \beta^{p^{\prime}-1} .
$$

Similarly using the fact that $2^{n}|\gamma|^{-1 / 2} \leq \alpha \beta^{p^{\prime}-1}$, we get that

$$
\left\|K_{N, \gamma}(x, t) \sum_{\mathcal{S}_{m}} 2^{-j} p_{k, 0}(t)\right\|_{L_{x, y, t}^{\infty}} \lesssim \sup _{k} \sum_{j:(j, k) \in \mathcal{S}_{m}} 2^{-j-k} 2^{2 n}|\gamma|^{-1 / 2} \lesssim C^{-1} \alpha \beta^{p^{\prime}-1} .
$$

This proves that $K_{n, \gamma ; \alpha, \beta}^{1}$ satisfies the right bound in (3.14).

It remains to prove that

$$
K_{N, \gamma ; \alpha, \beta}^{2}(x, y, t)=\chi(t) \sum_{\mathcal{S}_{m}}\left[p_{k, j}(t)-\rho_{k, j} p_{k, 0}(t)\right] K_{N, \gamma}(x, y, t)
$$

satisfies the appropriate estimates in (3.14). Here we need the following lemma from [34]:

Lemma 3.4. Assume $\kappa>0, Q, M \in \mathbb{N}^{*}, M \geq 8 Q, S \subseteq\{1, \ldots, Q\}$, and $\eta: \mathbb{R} \rightarrow[0,1]$ is a smooth function supported in $[-2,2]$. Then, for any $t \in \mathbb{R}$,

$$
\sum_{q \in S, a \in \mathbb{Z}(a, q)=1} \eta(M Q(t-a / q))=\sum_{m \in \mathbb{Z}}(M Q)^{-1} \widehat{\eta}(2 \pi m /(M Q)) c_{m} e^{2 \pi i m t},
$$

and the coefficients $c_{m}$ have the properties

$$
c_{m}=\sum_{q \in S, 0 \leq a \leq q-1,(a, q)=1} e^{-2 \pi i m \cdot a / q}, \quad \sup _{m \in \mathbb{Z}}\left|c_{m}\right| \leq 4 Q^{2}, \quad\left|c_{m}\right| \leq C_{\kappa} d(m, Q) Q^{1+\kappa},
$$

where $d(m, Q)$ denotes the number of divisors of $m$ less than or equal to $Q$.

Using this lemma and because of our definition of $\rho_{k, j}$ in (3.19), we can write

$$
p_{k, j}(t)-\rho_{k, j} p_{k, 0}(t)=\sum_{m \in \mathbb{Z}} c_{m} e^{i 2 \pi m t} 2^{-j-k-n-10}\left[\hat{\phi}_{1}\left(2^{-j-k-n-10} 2 \pi m\right)-\hat{\phi}_{1}\left(2^{-k-n-10} 2 \pi m\right)\right]
$$

if $k<n-k$, and

$$
p_{k, n-k}(t)-\rho_{k, n-k} p_{k, 0}(t)=\sum_{m \in \mathbb{Z}} c_{m} e^{i 2 \pi m t} 2^{-2 n-10}\left[\widehat{\phi_{\geq 1}}\left(2^{-2 n-10} 2 \pi m\right)-2 \hat{\phi}_{1}\left(2^{-k-n-10} 2 \pi m\right)\right]
$$


In either case, we have that for $0 \leq k \leq n-10,0 \leq j \leq n-k$ :

$$
p_{k, j}(t)-\rho_{k, j} p_{k, 0}(t)=\sum_{m \in \mathbb{Z}} b_{m} e^{i 2 \pi m t}
$$

with

$$
b_{0}=0, \quad\left|b_{m}\right| \leq C_{\varepsilon} d\left(m, 2^{k+1}\right) 2^{-j-n+\varepsilon k}\left(1+2^{-j-k-n}|m|\right)^{-10},
$$

where $d(m, Q)$ denotes the number of divisors of $m$ less than $Q$. We remark that $\rho_{k, j}$ were specifically introduced to make $b_{0}=0$ (recall that $\widehat{\phi_{\geq 1}}(0)=2 \widehat{\phi_{1}}(0)$ ).

Using the fact that $d(m, Q) \lesssim C_{\varepsilon}|m|^{\varepsilon}$, we finally get

$$
\begin{aligned}
\left\|\mathcal{F}_{x, y, t} K_{N, \gamma ; \alpha, \beta}^{2}\right\|_{L_{\xi, z, \tau}^{\infty}} & \lesssim \sum_{(k, j) \in \mathcal{S}_{m}} \sup _{\xi, z, \tau}\left|\int_{\mathbb{R}} \chi(t)\left(p_{k, j}(t)-2^{-j} p_{k, 0}(t)\right) e^{i t\left[|\xi|^{2}+|z|^{2}-\tau-\gamma\right]} d t\right| \\
& \leq \sum_{(k, j) \in \mathcal{S}_{m}} \sum_{m \in \mathbb{Z}}\left|b_{m}\right| \lesssim C_{\varepsilon} \sum_{(k, j) \in \mathcal{S}_{m}} 2^{\varepsilon n} 2^{-j-n} .
\end{aligned}
$$

which gives second bound in (3.14). This finishes the proof.

Remark 3.5. One can sharpen the estimate (3.2) to allow for different smoothness in both directions, namely, when $1 \leq M \leq N$,

$$
\left\|P_{\leq N} P_{\leq M}^{x} e^{i t \Delta_{\mathbb{R} \times \mathbb{T}^{2}}} u_{0}\right\|_{l_{\gamma}^{q} L_{x, y, t}^{p}\left(\mathbb{R} \times \mathbb{T}^{2} \times[2 \pi \gamma, 2 \pi(\gamma+1)]\right)} \lesssim\left(\frac{M}{N}\right)^{\theta} N^{\left(\frac{3}{2}-\frac{5}{p}\right)}\left\|u_{0}\right\|_{L_{x, y}^{2}\left(\mathbb{R} \times \mathbb{T}^{2}\right)} .
$$

where $\theta=1 / 2-2 / p$.

This follows directly from (3.4) after one refines the diagonal contribution (3.5) with

$$
\left\|\int_{\mathbb{R}} e^{-i s \Delta_{\mathbb{R} \times \mathbb{T}^{2}}} P_{\leq N} P_{\leq M}^{x} h(s) d s\right\|_{L_{x, y}^{2}\left(\mathbb{R} \times \mathbb{T}^{2}\right)} \lesssim\left(\frac{M}{N}\right)^{\frac{1}{2}-\frac{1}{p}} N^{\frac{3}{2}-\frac{5}{p}}\|h\|_{L_{x, y, t}^{p^{\prime}}\left(\mathbb{R} \times \mathbb{T}^{2} \times[-2 \pi, 2 \pi]\right)} .
$$

The claim follow: 10 .

\section{LOCAL WELL-POSEDNESS AND SMALL-DATA SCATTERING}

We start by translating the Strichartz estimate in Theorem 3.1 into an embedding theorem for $U_{\Delta}^{p}$ spaces:

Lemma 4.1. For $p>4$ and $q$ as in Theorem [3.1, the following estimate holds for any time interval $I \subset \mathbb{R}$ and every cube $Q \subset \mathbb{R}^{3}$ of size $N$ :

$$
\left\|\mathbb{1}_{I}(t) P_{Q} u\right\|_{l_{\gamma}^{q} L_{x, y, t}^{p}\left(\mathbb{R} \times \mathbb{T}^{2} \times I_{\gamma}\right)} \lesssim N^{\frac{3}{2}-\frac{5}{p}}\|u\|_{U_{\Delta}^{\min (p, q)}\left(I ; L_{x, y}^{2}\left(\mathbb{R} \times \mathbb{T}^{2}\right)\right)}
$$

The proof of this lemma is slightly tedious but straightforward. We omit the details.

\footnotetext{
${ }^{10}$ Taking into account the better derivative gain in the non-diagonal part (by optimizing the power of $N$ in (3.15) ), one can improve $\theta$ in (3.24) into $\theta=1-\frac{4}{p}$ if $4<p<6$ and $1 / 2-1 / p+\varepsilon$ if $p \geq 6$.
} 
4.1. Contraction argument. The following quantity will be of use in the local existence theory:

$$
\|u\|_{Z^{\prime}(I)}=\|u\|_{X^{1}(I)}^{1 / 4}\|u\|_{Z(I)}^{3 / 4}
$$

The local well-posedness theory will follow from the following two lemmas:

Lemma 4.2. Suppose that $u_{i}=P_{N_{i}} u_{i}$ for $i=1,2,3$ satisfying $N_{1} \geq N_{2} \geq N_{3}$. There exists $\delta>0$ such that the following estimate holds for any interval $I \subset \mathbb{R}$ :

$$
\left\|u_{1} u_{2} u_{3}\right\|_{L_{x, y, t}^{2}\left(\mathbb{R} \times \mathbb{T}^{2} \times I\right)} \lesssim\left(\frac{N_{3}}{N_{1}}+\frac{1}{N_{2}}\right)^{\delta}\left\|u_{1}\right\|_{Y^{0}(I)}\left\|u_{2}\right\|_{Z^{\prime}(I)}\left\|u_{3}\right\|_{Z^{\prime}(I)}
$$

Proof. It is sufficient to study the case $I=\mathbb{R}$. The result is a consequence of the following two estimates:

$$
\left\|u_{1} u_{2} u_{3}\right\|_{L_{x, y, t}^{2}\left(\mathbb{R} \times \mathbb{T}^{2} \times \mathbb{R}\right)} \lesssim N_{2} N_{3}\left(\frac{N_{3}}{N_{1}}+\frac{1}{N_{2}}\right)^{\delta_{0}}\left\|u_{1}\right\|_{Y^{0}(\mathbb{R})}\left\|u_{2}\right\|_{Y^{0}(\mathbb{R})}\left\|u_{3}\right\|_{Y^{0}(\mathbb{R})} .
$$

and

$$
\left\|u_{1} u_{2} u_{3}\right\|_{L_{x, y, t}^{2}\left(\mathbb{R} \times \mathbb{T}^{2} \times \mathbb{R}\right)} \lesssim\left\|u_{1}\right\|_{Y^{0}(\mathbb{R})}\left\|u_{2}\right\|_{Z(\mathbb{R})}\left\|u_{3}\right\|_{Z(\mathbb{R})}
$$

Estimate (4.4) follows as in Proposition 3.5 of 31 ] or Proposition 2.8 of 32 . As for (4.5), we first notice that, by orthogonality considerations, we may replace $u_{1}$ by $P_{C} u_{1}$ where $C$ is a cube of dimension $N_{2}$. This allows to estimate:

$$
\begin{aligned}
& \left\|\left(P_{C} u_{1}\right) u_{2} u_{3}\right\|_{L_{x, y, t}^{2}\left(\mathbb{R} \times \mathbb{T}^{2} \times \mathbb{R}\right)} \lesssim\left\|P_{C} u_{1}\right\|_{l_{\gamma}^{\frac{36}{5}} L_{x, y, t}^{\frac{9}{2}}}\left\|u_{2}\right\|_{l_{\gamma}^{\frac{36}{5}} L_{x, y, t}^{9 / 2}}\left\|u_{3}\right\|_{l_{\gamma}^{\frac{9}{2}} L_{x, y, t}^{18}} \\
& \lesssim N_{2}^{\frac{7}{18}}\left\|P_{C} u_{1}\right\|_{Y^{0}}\left\|u_{2}\right\|_{l_{\gamma}^{\frac{36}{5}} L_{x, y, t}^{9 / 2}}\left\|u_{3}\right\|_{l_{\gamma}^{\frac{9}{2}}} L_{x, y, t}^{18} \\
& \lesssim\left(\frac{N_{3}}{N_{2}}\right)^{2 / 9}\left\|P_{C} u_{1}\right\|_{Y^{0}}\left\|u_{2}\right\|_{Z(\mathbb{R})}\left\|u_{3}\right\|_{Z(\mathbb{R})}
\end{aligned}
$$

which is acceptable.

As a result of the above lemma, we have:

Lemma 4.3. For $u_{i} \in X^{1}(I), i=1, \ldots, 5$, there holds that

$$
\left\|\sum_{K \geq 1} P_{K} u_{1} \prod_{i=2}^{5} P_{\leq C K} \tilde{u}_{i}\right\|_{N(I)} \lesssim_{C}\left\|u_{1}\right\|_{X^{1}(I)} \prod_{i=2}^{5}\left\|u_{i}\right\|_{Z^{\prime}(I)} .
$$

and in particular,

$$
\left\|\prod_{i=1}^{5} \tilde{u}_{i}\right\|_{N(I)} \lesssim \sum_{j=1}^{5}\left\|u_{j}\right\|_{X^{1}(I)} \prod_{i \neq j}\left\|u_{i}\right\|_{Z^{\prime}(I)}
$$

where $\tilde{u}_{i}$ is either $u_{i}$ or $\overline{u_{i}}$.

Proof. The proof is a fairly standard consequence of Lemma 4.2 (cf. Lemma 3.2 of [34]).

We are now ready to prove the local existence theory for large data and global existence and scattering theory for small data. The proof of the following statement is very similar from the proofs in [34, Section $3]$ given Lemma 4.3 and is omitted. 
Proposition 4.4 (Local Well-posedness). Let $E>0$ and suppose that $\left\|u_{0}\right\|_{H^{1}\left(\mathbb{R} \times \mathbb{T}^{2}\right)} \leq E$. There exists $\delta_{0}=\delta_{0}(E) \approx \min \left(1, E^{-1}\right)$ such that if

$$
\left\|e^{i t \Delta_{\mathbb{R} \times \mathbb{T}^{2}}} u_{0}\right\|_{Z(I)} \leq \delta \leq \delta_{0}
$$

on some interval $I \subset \mathbb{R}$ containing 0 , then there exists a unique strong solution $u \in X_{c}^{1}(I)$ of (1.1) satisfying $u(0)=u_{0}$ and

$$
\left\|u(t)-e^{i t \Delta_{\mathbb{R} \times \mathbb{T}^{2}}} u_{0}\right\|_{X^{1}(I)} \lesssim E^{2} \delta^{3} .
$$

Moreover, the quantities $E(u)$ and $M(u)$ defined in (1.4) are conserved on $I$.

Observe that if $u \in X_{c}^{1}(\mathbb{R})$, then $u$ scatters as $t \rightarrow \pm \infty$ as in (1.3). Also, if $E$ is small enough, $I$ can be taken to be $\mathbb{R}$ which proves Theorem 1.1 .

Lemma 4.5 (Controlling norm). Suppose that $u \in X_{c, \text { loc }}^{1}(I)$ is a strong solution on an interval $I \subset \mathbb{R}$ satisfying:

$$
\|u\|_{Z(I)}<+\infty .
$$

(1) If I is finite, then $u$ can be extended as a strong solution in $X_{c}^{1}\left(I^{\prime}\right)$ on a strictly larger interval $I^{\prime}, I \subsetneq I^{\prime} \subset \mathbb{R}$. In particular, if u blows up in finite time, then the $Z$ norm of $u$ has to blow up.

(2) If I is infinite, then $u \in X_{c}^{1}(I)$.

Finally, we end this section with the following stability result:

Proposition 4.6. Let $I \subset \mathbb{R}$ be an interval, and let $\tilde{u} \in X^{1}(I)$ solve the approximate equation:

$$
\left(i \partial_{t}+\Delta_{\mathbb{R} \times \mathbb{T}^{2}}\right) \tilde{u}=\rho|\tilde{u}|^{4} \tilde{u}+e
$$

where $\rho$ is a constant in $[0,1]$. Assume that the following boundedness estimates holds:

$$
\|\tilde{u}\|_{Z(I)}+\|\tilde{u}\|_{L_{t}^{\infty}\left(I, H^{1}\left(\mathbb{R} \times \mathbb{T}^{2}\right)\right)} \leq M .
$$

There exists $\epsilon_{0}=\epsilon_{0}(M) \in(0,1]$ such that if for some $t_{0} \in I$ :

$$
\left\|\tilde{u}\left(t_{0}\right)-u_{0}\right\|_{H^{1}\left(\mathbb{R} \times \mathbb{T}^{2}\right)}+\|e\|_{N(I)} \leq \epsilon<\epsilon_{0},
$$

then there exists a solution $u(t)$ to the exact equation:

$$
\left(i \partial_{t}+\Delta_{\mathbb{R} \times \mathbb{T}^{2}}\right) u=\rho|u|^{4} u
$$

with initial data $u_{0}$ which satisfies

$$
\begin{aligned}
\|u\|_{X^{1}(I)}+\|\tilde{u}\|_{X^{1}(I)} & \leq C(M) \\
\|u-\tilde{u}\|_{X^{1}(I)} & \leq C(M) \epsilon .
\end{aligned}
$$

\section{Nonlinear ANALysis OF PROFILES}

In this section, we describe and analyze the main profiles that appear in our linear and nonlinear profile decomposition. 
5.1. Euclidean profiles. The Euclidean profiles define a regime where we can compare solutions of quintic NLS on $\mathbb{R}^{3}$ with those on $\mathbb{R} \times \mathbb{T}^{2}$. Of course, this analysis is only possible in the small-scale limit, i.e. for solutions that concentrate at a point. Our arguments below follow closely those in [33, 34].

We fix a spherically-symmetric function $\eta \in C_{0}^{\infty}\left(\mathbb{R}^{3}\right)$ supported in the ball of radius 2 and equal to 1 in the ball of radius 1 . Given $\phi \in \dot{H}^{1}\left(\mathbb{R}^{3}\right)$ and a real number $N \geq 1$ we define

$$
\begin{aligned}
\phi_{N} \in H^{1}\left(\mathbb{R}^{3}\right), & \phi_{N}(x)=N^{\frac{1}{2}} \eta\left(N^{\frac{1}{2}} x\right) \phi(N x), \\
f_{N} \in H^{1}\left(\mathbb{R} \times \mathbb{T}^{2}\right), & f_{N}(y)=\phi_{N}\left(\Psi^{-1}(y)\right),
\end{aligned}
$$

where $\Psi:\left\{x \in \mathbb{R}^{3}:|x|<1\right\} \rightarrow O_{0} \subseteq \mathbb{R} \times \mathbb{T}^{2}, \Psi(x)=x$. Thus $\phi_{N}$ is an $\dot{H}^{1}$-invariant rescaling of a smooth compact truncation $\phi$, and $f_{N}$ is the function obtained by transferring $\phi_{N}$ to a neighborhood of 0 in $\mathbb{R} \times \mathbb{T}^{2}$. Notice that

$$
\left\|f_{N}\right\|_{H^{1}\left(\mathbb{R} \times \mathbb{T}^{2}\right)} \lesssim\|\phi\|_{\dot{H}^{1}\left(\mathbb{R}^{3}\right)}
$$

We define also

$$
E_{\mathbb{R}^{3}}(\phi)=\frac{1}{2} \int_{\mathbb{R}^{3}}\left|\nabla_{\mathbb{R}^{3}} \phi\right|^{2} d x+\frac{1}{6} \int_{\mathbb{R}^{3}}|\phi|^{6} d x .
$$

We will use the main theorem of [18, Theorem 1.1] (see also [42]), in the following form.

Theorem 5.1. Assume $\psi \in \dot{H}^{1}\left(\mathbb{R}^{3}\right)$. Then there is a unique global solution $v \in C\left(\mathbb{R}: \dot{H}^{1}\left(\mathbb{R}^{3}\right)\right)$ of the initial-value problem

$$
\left(i \partial_{t}+\Delta_{\mathbb{R}^{3}}\right) v=v|v|^{4}, \quad v(0)=\psi
$$

and

$$
\left\|\left|\nabla_{\mathbb{R}^{3}} v\right|\right\|_{\left(L_{t}^{\infty} L_{x}^{2} \cap L_{t}^{2} L_{x}^{6}\right)\left(\mathbb{R}^{3} \times \mathbb{R}\right)} \leq \widetilde{C}\left(E_{\mathbb{R}^{3}}(\psi)\right) .
$$

Moreover this solution scatters in the sense that there exists $\psi^{ \pm \infty} \in \dot{H}^{1}\left(\mathbb{R}^{3}\right)$ such that

$$
\left\|v(t)-e^{i t \Delta_{\mathbb{R}^{3}}} \psi^{ \pm \infty}\right\|_{\dot{H}^{1}\left(\mathbb{R}^{3}\right)} \rightarrow 0
$$

as $t \rightarrow \pm \infty$. Besides, if $\psi \in H^{5}\left(\mathbb{R}^{3}\right)$ then $v \in C\left(\mathbb{R}: H^{5}\left(\mathbb{R}^{3}\right)\right)$ and

$$
\sup _{t \in \mathbb{R}}\|v(t)\|_{H^{5}\left(\mathbb{R}^{3}\right)} \lesssim\|\psi\|_{H^{5}\left(\mathbb{R}^{3}\right)} 1 .
$$

Our first result in this section is the following lemma:

Lemma 5.2. Assume $\phi \in \dot{H}^{1}\left(\mathbb{R}^{3}\right), T_{0} \in(0, \infty)$, and $\rho \in\{0,1\}$ are given, and define $f_{N}$ as in (5.1). Then the following conclusions hold:

(i) There is $N_{0}=N_{0}\left(\phi, T_{0}\right)$ sufficiently large such that for any $N \geq N_{0}$ there is a unique solution $U_{N} \in C\left(\left(-T_{0} N^{-2}, T_{0} N^{-2}\right) ; H^{1}\left(\mathbb{R} \times \mathbb{T}^{2}\right)\right)$ of the initial-value problem

$$
\left(i \partial_{t}+\Delta\right) U_{N}=\rho U_{N}\left|U_{N}\right|^{4}, \quad U_{N}(0)=f_{N} .
$$

Moreover, for any $N \geq N_{0}$,

$$
\left\|U_{N}\right\|_{X^{1}\left(-T_{0} N^{-2}, T_{0} N^{-2}\right)} \lesssim_{E_{\mathbb{R}^{3}}(\phi)} 1 .
$$

(ii) Assume $\varepsilon_{1} \in(0,1]$ is sufficiently small (depending only on $\left.E_{\mathbb{R}^{3}}(\phi)\right)$, $\phi^{\prime} \in H^{5}\left(\mathbb{R}^{3}\right)$, and $\left\|\phi-\phi^{\prime}\right\|_{\dot{H}^{1}\left(\mathbb{R}^{3}\right)} \leq$ $\varepsilon_{1}$. Let $v^{\prime} \in C\left(\mathbb{R}: H^{5}\right)$ denote the solution of the initial-value problem

$$
\left(i \partial_{t}+\Delta_{\mathbb{R}^{3}}\right) v^{\prime}=\rho v^{\prime}\left|v^{\prime}\right|^{4}, \quad v^{\prime}(0)=\phi^{\prime} .
$$


For $R \geq 1$, and $N \geq 10 R$ we define

$$
\begin{array}{lc}
v_{R}^{\prime}(x, t)=\eta(x / R) v^{\prime}(x, t), & (x, t) \in \mathbb{R}^{3} \times\left(-T_{0}, T_{0}\right), \\
v_{R, N}^{\prime}(x, t)=N^{\frac{1}{2}} v_{R}^{\prime}\left(N x, N^{2} t\right), & (x, t) \in \mathbb{R}^{3} \times\left(-T_{0} N^{-2}, T_{0} N^{-2}\right), \\
V_{R, N}(y, t)=v_{R, N}^{\prime}\left(\Psi^{-1}(y), t\right) & (y, t) \in \mathbb{R} \times \mathbb{T}^{2} \times\left(-T_{0} N^{-2}, T_{0} N^{-2}\right) .
\end{array}
$$

Then there is $R_{0} \geq 1$ (depending on $T_{0}$ and $\phi^{\prime}$ and $\varepsilon_{1}$ ) such that, for any $R \geq R_{0}$ and $N \geq 10 R$,

$$
\limsup _{N \rightarrow \infty}\left\|U_{N}-V_{R, N}\right\|_{X^{1}\left(-T_{0} N^{-2}, T_{0} N^{-2}\right)} \lesssim_{E_{\mathbb{R}^{3}}(\phi)} \varepsilon_{1}
$$

Proof. The proof of Lemma 5.2 is very similar to the proof of [33, Lemma 4.2].

To understand linear and nonlinear evolutions beyond the Euclidean window we need an additional extinction lemma:

Lemma 5.3. Suppose that $\phi \in \dot{H}^{1}\left(\mathbb{R}^{3}\right), \epsilon>0$, and $I \subset \mathbb{R}$ is an interval. Assume that

$$
\|\phi\|_{\dot{H}^{1}\left(\mathbb{R}^{3}\right)} \leq 1, \quad\left\|\nabla_{x} e^{i t \Delta} \phi\right\|_{L_{t}^{2} L_{x}^{6}\left(I \times \mathbb{R}^{3}\right)} \leq \epsilon .
$$

For $N \geq 1$, we define as before:

$$
Q_{N} \phi=\eta\left(N^{-1 / 2} x\right) \phi(x), \quad \phi_{N}=N^{1 / 2} Q_{N}(N x)=N^{1 / 2} \eta\left(N^{1 / 2} x\right) \phi(N x), \quad f_{N}(y)=\phi_{N}\left(\Psi^{-1}(y)\right) .
$$

Then there exists $N_{0}=N_{0}(\phi, \epsilon)$ such that for any $N \geq N_{0}$,

$$
\left\|e^{i t \Delta} f_{N}\right\|_{Z\left(N^{-2} I\right)} \lesssim \epsilon^{\frac{1}{6}}
$$

Proof. The proof is fairly similar to that of Lemma of 4.3 [34], so we omit the details for brevity.

We are now ready to describe the nonlinear solutions of (1.1) corresponding to data concentrating at a point. Let $\widetilde{\mathcal{F}}_{e}$ denote the set of renormalized Euclidean frame:11

$$
\begin{aligned}
\widetilde{\mathcal{F}}_{e}:=\left\{\left(N_{k}, t_{k}, x_{k}\right)_{k \geq 1}:\right. & N_{k} \in[1, \infty), x_{k} \in \mathbb{R} \times \mathbb{T}^{2}, N_{k} \rightarrow \infty, \\
& \text { and either } \left.t_{k}=0 \text { for any } k \geq 1 \text { or } \lim _{k \rightarrow \infty} N_{k}^{2}\left|t_{k}\right|=\infty\right\} .
\end{aligned}
$$

Given $f \in L_{x, y}^{2}\left(\mathbb{R} \times \mathbb{T}^{2}\right), t_{0} \in \mathbb{R}$, and $x_{0} \in \mathbb{R} \times \mathbb{T}^{2}$, we define:

$$
\pi_{x_{0}} f=f\left(x-x_{0}\right), \quad \Pi_{\left(t_{0}, x_{0}\right)} f=\left(e^{-i t_{0} \Delta_{\mathbb{R} \times \mathbb{T}^{2}}} f\right)\left(x-x_{0}\right)=\pi_{x_{0}} e^{-i t_{0} \Delta_{\mathbb{R} \times \mathbb{T}^{2}}} f .
$$

Also for $\phi \in \dot{H}^{1}\left(\mathbb{R}^{3}\right)$ and $N \geq 1$, we denote the function obtained in (5.1) by:

$$
T_{N}^{e} \phi:=N^{1 / 2} \widetilde{\phi}\left(N \Psi^{-1}(x)\right) \quad \text { where } \quad \widetilde{\phi}(y):=\eta\left(y / N^{1 / 2}\right) \phi(y),
$$

and observe as before that $T_{N}^{e}: \dot{H}^{1}\left(\mathbb{R}^{3}\right) \rightarrow H^{1}\left(\mathbb{R} \times \mathbb{T}^{2}\right)$ with $\left\|T_{N}^{e} \phi\right\|_{H^{1}\left(\mathbb{R} \times \mathbb{T}^{2}\right)} \lesssim\|\phi\|_{\dot{H}^{1}\left(\mathbb{R}^{3}\right)}$.

\footnotetext{
${ }^{11}$ We will later consider a slightly more general class of frames, called Euclidean frames, see Definition 6.1 However, for all practical purposes (cf. Lemma 6.2), it suffices to prove Proposition 5.4 under the stronger assumption that $\mathcal{O}$ is a renormalized Euclidean frame.
} 
Proposition 5.4. Assume that $\mathcal{O}=\left(N_{k}, t_{k}, x_{k}\right)_{k} \in \widetilde{\mathcal{F}}_{e}, \phi \in \dot{H}^{1}\left(\mathbb{R}^{3}\right)$, and let $U_{k}(0)=\Pi_{t_{k}, x_{k}}\left(T_{N_{k}}^{e} \phi\right)$ :

(i) For $k$ large enough (depending only on $\phi, \mathcal{O}$ ), there is a nonlinear solution $U_{k} \in X_{c}^{1}(\mathbb{R})$ of the equation (1.1) satisfying:

$$
\left\|U_{k}\right\|_{X^{1}(\mathbb{R})} \lesssim E_{\mathbb{R}^{3}(\phi)} 1 .
$$

(ii) There exists a Euclidean solution $u \in C\left(\mathbb{R}: \dot{H}^{1}\left(\mathbb{R}^{3}\right)\right)$ of

$$
\left(i \partial_{t}+\Delta_{\mathbb{R}^{3}}\right) u=|u|^{4} u
$$

with scattering data $\phi^{ \pm \infty}$ defined as in (5.4) such that the following holds, up to a subsequence: for any $\varepsilon>0$, there exists $T(\phi, \varepsilon)$ such that for all $T \geq T(\phi, \varepsilon)$ there exists $R(\phi, \varepsilon, T)$ such that for all $R \geq R(\phi, \varepsilon, T)$, there holds that

$$
\left\|U_{k}-\tilde{u}_{k}\right\|_{X^{1}\left(\left\{\left|t-t_{k}\right| \leq T N_{k}^{-2}\right\}\right)} \leq \varepsilon
$$

for $k$ large enough, where

$$
\left(\pi_{-x_{k}} \tilde{u}_{k}\right)(x, t)=N_{k}^{\frac{1}{2}} \eta\left(N_{k} \Psi^{-1}(x) / R\right) u\left(N_{k} \Psi^{-1}(x), N_{k}^{2}\left(t-t_{k}\right)\right) .
$$

In addition, up to a subsequence,

$$
\left\|U_{k}(t)-\Pi_{t_{k}-t, x_{k}} T_{N_{k}}^{e} \phi^{ \pm \infty}\right\|_{X^{1}\left(\left\{\left(t-t_{k}\right) \geq T N_{k}^{-2}\right\}\right)} \leq \varepsilon,
$$

for $k$ large enough (depending on $\phi, \varepsilon, T, R$ ).

Proof. cf. Proposition 4.4 of [34] for a similar argument.

5.2. Large Scale Profiles. In this subsection, we analyze the large-scale profiles that appear in the profile decomposition in Section [6. We need the following notation: given $\psi \in H^{0,1}\left(\mathbb{R} \times \mathbb{T}^{2}\right)$ and $M \leq 1$, we define the large-scale rescaling

$$
T_{M}^{l s} \psi(x, y):=M^{\frac{1}{2}} \widetilde{\psi^{*}}(M x, y) \quad \text { where } \quad \widetilde{\psi}^{*}(x, y)=P_{\leq M^{-1 / 100}}^{x} \psi(x, y) .
$$

It's easy to see that,

$$
T_{M}^{l s}: H^{0,1}\left(\mathbb{R} \times \mathbb{T}^{2}\right) \rightarrow H^{1}\left(\mathbb{R} \times \mathbb{T}^{2}\right) \text { is a linear operator with }\left\|T_{M}^{l s} \psi\right\|_{H^{1}\left(\mathbb{R} \times \mathbb{T}^{2}\right)} \lesssim\|\psi\|_{H^{0,1}\left(\mathbb{R} \times \mathbb{T}^{2}\right)} .
$$

The purpose of this section is to understand the behavior of nonlinear solutions $u_{k}$ of (1.1) with initial data as above and variations thereof. As we will see these are tightly connected to solutions of the quintic resonant system defined in (1.6) that we recall here for convenience.

5.2.1. The quintic resonant system. We consider the quintic resonant system

$$
\begin{aligned}
\left(i \partial_{t}+\partial_{x x}\right) u_{j}= & \sum_{\left(p_{1}, p_{2}, p_{3}, p_{4}, p_{5}\right) \in \mathcal{R}(j)} u_{p_{1}} \bar{u}_{p_{2}} u_{p_{3}} \bar{u}_{p_{4}} u_{p_{5}}, \quad j \in \mathbb{Z}^{2} \\
\mathcal{R}(j)= & \left\{\vec{p}:=\left(p_{1}, p_{2}, p_{3}, p_{4}, p_{5}\right) \in\left(\mathbb{Z}^{2}\right)^{5}: p_{1}-p_{2}+p_{3}-p_{4}+p_{5}-j=0,\right. \\
& \left.\left|p_{1}\right|^{2}-\left|p_{2}\right|^{2}+\left|p_{3}\right|^{2}-\left|p_{4}\right|^{2}+\left|p_{5}\right|^{2}-|j|^{2}=0\right\} .
\end{aligned}
$$

with initial data $\vec{u}(0)=\left\{u_{j}(0)\right\}_{j \in \mathbb{Z}^{2}} \in h^{1} L^{2}$, where $h^{1} L^{2}$ is defined in (2.2). In light of the conservation laws (8.2) proved in the Appendix, the following energy:

$$
E_{l s}[\vec{u}]:=\sum_{p \in \mathbb{Z}^{2}}\left[1+|p|^{2}\right]\left\|u_{p}\right\|_{L_{x}^{2}(\mathbb{R})}^{2}
$$

is conserved and hence so is the $h^{1} L^{2}$ norm of any solution of (1.6). 
We remark that in the special case when only one component of the initial data is nontrivial: $u_{j}(0)=$ $0, j \neq 0$, this is propagated by the nonlinear flow and (1.6) reduces to the scalar defocusing mass-critical Schrödinger equation,

$$
\left(i \partial_{t}+\partial_{x x}\right) u_{0}=\left|u_{0}\right|^{4} u_{0}, \quad E_{l s}\left(u_{0}\right)=\left\|u_{0}\right\|_{L_{x}^{2}(\mathbb{R})}^{2} .
$$

Based on the local and small data theory that we develop in the appendix and the large-data scattering result in the scalar case 21, we conjecture the following global existence theorem about solutions to (1.6):

Conjecture 5.5. Any initial data $\vec{u}_{0}$ of finite $E_{l s}$ energy leads to a global solution of (1.6) satisfying

$$
\|\vec{u}\|_{\vec{W}}^{2}:=\sum_{p \in \mathbb{Z}^{2}}\left[1+|p|^{2}\right]\left\|u_{p}\right\|_{L_{x, t}^{6}\left(\mathbb{R}_{x} \times \mathbb{R}_{t}\right)}^{2} \leq \Lambda_{l s}\left(E_{l s}(\vec{u})\right)
$$

for some nondecreasing finite function $\Lambda_{l s}$. In addition, this solution scatters in the sense that there exists $\vec{v}^{ \pm \infty} \in h^{1} L^{2}$ such that

$$
\sum_{p \in \mathbb{Z}^{2}}\left[1+|p|^{2}\right]\left\|u_{p}(t)-e^{i t \partial_{x x}} v_{p}^{ \pm \infty}\right\|_{L_{x}^{2}(\mathbb{R})}^{2} \rightarrow 0 \quad \text { as } t \rightarrow \pm \infty .
$$

As we also show in the appendix, this conjecture is in fact implied by the conclusion of Theorem 1.3 In addition, the local wellposedness theory for (1.6) (cf. Appendix) gives that this conjecture is true under the smallness hypothesis $E_{l s}(\vec{u})<\delta$ for some $\delta>0$. Finally, the result of Dodson 21] shows that this conjecture is again true if one adds the additional assumption that $\vec{u}(0)$ is, in fact scalar.

A direct consequence of Conjecture 5.5 and the propagation of regularity part of the local well-posedness proposition 8.1 is the following:

Proposition 5.6. Assume that Conjecture 5.5 holds true. Suppose that $\vec{u}_{0} \in h^{1} L^{2}$ and that $\vec{u} \in C(\mathbb{R}$ : $\left.h^{1} L^{2}\right)$ is the solution of (1.6) with initial data $\vec{u}_{0}$ given by Conjecture 5.5. Suppose also that $\vec{v}_{0} \in h^{5} H^{4}$ satisfies

$$
\left\|\vec{u}_{0}-\vec{v}_{0}\right\|_{h^{1} L^{2}} \lesssim \varepsilon
$$

and that $\vec{v}(t)$ is the solution to (1.6) with initial data $\vec{v}(0)=\vec{v}_{0}$. Then, it holds that:

$$
\begin{aligned}
\left\|\left(1-\partial_{x x}\right)^{2}\left\{\left(1+|p|^{4}\right) v_{p}\right\}_{p}\right\|_{L_{t}^{\infty}\left(h^{1} L^{2}\right) \cap \vec{W}(\mathbb{R})} & \lesssim\left\|\vec{u}_{0}\right\|_{h^{1} L^{2}} \\
\|\vec{u}-\vec{v}\|_{L_{t}^{\infty}\left(h^{1} L^{2}\right) \cap \vec{W}(\mathbb{R})} & \lesssim\left\|\vec{u}_{0}\right\|_{h^{1} L^{2}} \varepsilon .
\end{aligned}
$$

and there exists $\vec{w}^{ \pm} \in h^{5} H^{4}$ such that

$$
\sum_{p \in \mathbb{Z}^{2}}\left[1+|p|^{2}\right]\left\|v_{p}(t)-e^{i t \partial_{x x}} w_{p}^{ \pm}\right\|_{L_{x}^{2}(\mathbb{R})}^{2} \rightarrow 0 \text { as } t \rightarrow \pm \infty .
$$

5.2.2. Description of the large scale profiles. We are now in a position to describe the behavior of solutions with initial data $f_{M}=T_{M}^{l s} \psi$. We also define for $\vec{\psi}=\left\{\psi_{p}\right\}_{p}$ as in (2.3),

$$
\vec{\psi}_{M}:=\left\{\psi_{p}^{M}\right\}_{p}, \quad \text { where } \psi_{p}^{M}(x):=M^{\frac{1}{2}} \psi_{p}(M x),
$$

and, more generally, we similarly define $\vec{u}_{M}$ from $\vec{u}$ for any $\vec{u} \in h^{1} L^{2}$. Clearly, with $f_{M}$ as in (5.17),

$$
\left\|\vec{\psi}_{M}\right\|_{h^{1} L^{2}}=\|\psi\|_{H^{0,1}\left(\mathbb{R} \times \mathbb{T}^{2}\right)} ; \quad\left\|\vec{\psi}_{M}-\vec{f}_{M}\right\|_{h^{1} L^{2}} \rightarrow 0 \text { as } M \rightarrow 0 .
$$

Our first result in this direction is the following lemma: 
Lemma 5.7. Assume that Conjecture 5.5 holds true. Let $\psi \in H^{0,1}\left(\mathbb{R} \times \mathbb{T}^{2}\right), T_{0} \in(0, \infty)$, and $\rho \in\{0,1\}$ be given, and define $f_{M}=T_{M}^{l s} \psi(x, y)$ as in (5.17). The following conclusions hold:

(i) There is $M_{0}=M_{0}\left(\phi, T_{0}\right)$ sufficiently small such that for any $M \leq M_{0}$ there is a unique solution $U_{M} \in C\left(\left(-T_{0} M^{-2}, T_{0} M^{-2}\right) ; H^{1}\left(\mathbb{R} \times \mathbb{T}^{2}\right)\right)$ of the initial-value problem

$$
\left(i \partial_{t}+\Delta_{\mathbb{R} \times \mathbb{T}^{2}}\right) U_{M}=\rho\left|U_{M}\right|^{4} U_{M}, \quad U_{M}(0)=f_{M} .
$$

Moreover, for any $M \leq M_{0}$,

$$
\left\|U_{M}\right\|_{X^{1}\left(-T_{0} M^{-2}, T_{0} M^{-2}\right)} \lesssim_{E_{l s}(\psi)} 1 .
$$

(ii) Assume $\varepsilon_{1} \in(0,1]$ is sufficiently small (depending only on $E_{l s}(\psi)$ ), $\vec{v}_{0} \in h^{5} H^{4}$, and $\left\|\vec{\psi}-\vec{v}_{0}\right\|_{h^{1} L^{2}} \leq \varepsilon_{1}$. Let $\vec{v} \in C\left(\mathbb{R}: h^{5} H^{4}\right)$ denote the solution of the initial-value problem

$$
\left(i \partial_{t}+\partial_{x x}\right) v_{j}=\rho \sum_{\left(p_{1}, p_{2}, p_{3}, p_{4}, p_{5}\right) \in \mathcal{R}(j)} v_{p_{1}} \overline{v_{p_{2}}} v_{p_{3}} \overline{v_{p_{4}}} v_{p_{5}}, \quad v_{j}(0)=v_{0, j}, j \in \mathbb{Z}^{2} .
$$

For $M \geq 1$ we define

$$
\begin{array}{ll}
v_{j, M}(x, t)=M^{\frac{1}{2}} v_{j}\left(M x, M^{2} t\right), & (x, t) \in \mathbb{R} \times\left(-T_{0} M^{-2}, T_{0} M^{-2}\right), \\
V_{M}(x, y, t)=\sum_{q \in \mathbb{Z}^{2}} e^{-i t|q|^{2}} e^{i\langle y, q\rangle} v_{q, M}(x, t), & (x, y, t) \in \mathbb{R} \times \mathbb{T}^{2} \times\left(-T_{0} M^{-2}, T_{0} M^{-2}\right) .
\end{array}
$$

Then

$$
\limsup _{M \rightarrow 0}\left\|U_{M}-V_{M}\right\|_{X^{1}\left(-T_{0} M^{-2}, T_{0} M^{-2}\right)} \lesssim_{E_{l s}(\vec{\psi})} \varepsilon_{1} .
$$

The proof of Lemma 5.7 is the equivalent for large-scale profiles of the proof of [33, Lemma 4.2]. Here we handle the error caused by neglecting the non resonant terms by using a normal form transformation. This reduction to the analysis of the resonant part of the system seems to be relevant only for large scale profiles $(M \rightarrow 0)$ or in very special regimes.

Proof. When $\rho=0$, there is nothing to prove. It suffices to prove part (ii). All implicit constants are allowed to depend on $\|\psi\|_{H^{0,1}\left(\mathbb{R} \times \mathbb{T}^{2}\right)}$.

First, we remark that by (5.19) and Stichartz estimates on $\mathbb{R}$, we have that

$$
\sum_{q \in \mathbb{Z}^{2}}\langle q\rangle^{2}\left\|v_{q}\right\|_{L_{t}^{5} L_{x}^{10}}^{2} \lesssim E_{l s}(\vec{\psi}), \quad \sum_{q \in \mathbb{Z}^{2}}\langle q\rangle^{10}\left\|v_{q}\right\|_{L_{t}^{5} W_{x}^{4,10}}^{2} \lesssim_{E_{l s}(\vec{\psi})}\|\vec{v}\|_{h^{5} H^{4}}^{2}
$$

We now show that $V_{M}$ is an almost solution in the sense of Lemma 4.6.

$$
\begin{aligned}
\left(i \partial_{t}+\Delta_{\mathbb{R} \times \mathbb{T}^{2}}\right) V_{M}-\left|V_{M}\right|^{4} V_{M} & =-\sum_{q \in \mathbb{Z}^{2}} e^{-i t|q|^{2}} e^{i\langle y, q\rangle} \sum_{\vec{p} \in \mathcal{N} \mathcal{R}(q)} v_{p_{1}, M} \overline{v_{p_{2}, M}} v_{p_{3}, M} \overline{v_{p_{4}, M}} v_{p_{5}, M} e^{-i t \Phi_{q, \vec{p}}} \\
& =L H S \\
\Phi_{q, \vec{p}} & =\left|p_{1}\right|^{2}-\left|p_{2}\right|^{2}+\left|p_{3}\right|^{2}-\left|p_{4}\right|^{2}+\left|p_{5}\right|^{2}-|q|^{2}
\end{aligned}
$$

where

$$
\mathcal{N} \mathcal{R}(q)=\left\{\vec{p}=\left(p_{1}, p_{2}, p_{3}, p_{4}, p_{5}\right): p_{1}-p_{2}+p_{3}-p_{4}+p_{5}-q=0 ; \Phi_{q, \vec{p}} \neq 0\right\}
$$

We now claim that, for $I=\left(-T_{0} M^{-2}, T_{0} M^{-2}\right)$,

$$
\|L H S\|_{N^{1}(I)} \lesssim\left\|\vec{v}_{0}\right\|_{h^{5} H^{4}} M .
$$


We first decompose

$$
L H S=P_{\geq 2^{-10}}^{x} L H S+P_{<2^{-10}}^{x} L H S=P_{h i g h} L H S+P_{\text {low }} L H S .
$$

Using Bernstein's estimate, the definition of the $N$-norm and (2.7), we obtain that

$$
\begin{aligned}
\left\|P_{\geq 2^{-10}}^{x} L H S\right\|_{N^{1}(0, S)}^{2} & \lesssim\left\|P_{\geq^{-10}}^{x} \partial_{x} L H S\right\|_{N^{1}(0, S)}^{2}=\sum_{q \in \mathbb{Z}^{2}}\left\|e^{i\langle y, q\rangle} P_{\geq 2^{-10}}^{x} \partial_{x} \mathcal{F}_{y}(L H S)(q)\right\|_{N^{1}(0, S)}^{2} \\
& \lesssim \sum_{q \in \mathbb{Z}^{2}}\langle q\rangle^{-2}\left[\langle q\rangle^{2} \sum_{\vec{p} \in \mathcal{N} \mathcal{R}(q)}\left\|\partial_{x}\left\{v_{p_{1}, M} \overline{v_{p_{2}, M}} v_{p_{3}, M} \overline{v_{p_{4}, M}} v_{p_{5}, M}\right\}\right\|_{L_{t}^{1} H_{x}^{1}}\right]^{2} .
\end{aligned}
$$

Since $q \lesssim \max \left(p_{1}, p_{2}, p_{3}, p_{4}, p_{5}\right)$, we see that, for any $q$,

$$
\begin{aligned}
& \langle q\rangle^{2} \sum_{\vec{p} \in \mathcal{N} \mathcal{R}(q)}\left\|\partial_{x}\left\{v_{p_{1}, M} \overline{v_{p_{2}, M}} v_{p_{3}, M} \overline{v_{p_{4}, M}} v_{p_{5}, M}\right\}\right\|_{L_{t}^{1} H_{x}^{1}} \\
& \lesssim M \sum_{\vec{p} \in \mathcal{N} \mathcal{R}(q)}\left\langle p_{1}\right\rangle^{2}\left\|\left(\partial_{x} v_{p_{1}}\right)_{M}\right\|_{L_{t}^{5} W_{x}^{1,10} \Pi_{j=2}^{5}}\left\{\left\langle p_{j}\right\rangle^{2}\left\|v_{p_{j}, M}\right\|_{L_{t}^{5} W^{1,10}}\right\}
\end{aligned}
$$

Thus $P_{\text {high }} L H S$ is acceptable once we sum in $q$.

The contribution of $P_{\text {low }} L H S$ is bounded by recalling the definition of the $N^{1}$ norm in (2.5) and integrating the time integral by parts as follows:

$$
\begin{aligned}
& \int_{0}^{S} e^{i(S-\sigma) \Delta_{\mathbb{R} \times \mathbb{T}^{2}}} P_{l o w} L H S(\sigma) d \sigma \\
& =-\sum_{q \in \mathbb{Z}^{2}} \sum_{\vec{p} \in \mathcal{N} \mathcal{R}(q)} e^{-i S\left[|q|^{2}+\Phi_{q, \vec{p}}\right]} e^{i\langle y, q\rangle} \int_{0}^{S} e^{i(S-\sigma)\left[\partial_{x x}+\Phi_{q, \vec{p}}\right]} P_{l o w}\left(v_{p_{1}, M} \overline{v_{p_{2}, M}} v_{p_{3}, M} \overline{v_{p_{4}, M}} v_{p_{5}, M}\right) d \sigma \\
& =\sum_{q \in \mathbb{Z}^{2}} \sum_{\vec{p} \in \mathcal{N} \mathcal{R}(q)} e^{-i S\left[|q|^{2}+\Phi_{q, \vec{p}}\right]} e^{i\langle y, q\rangle} \times \\
& \left\{\left[i e^{i(S-\sigma)\left[\partial_{x x}+\Phi_{q, \vec{p}}\right]}\left(\partial_{x x}+\Phi_{q, \vec{p}}\right)^{-1} P_{l o w}\left(v_{p_{1}, M} \overline{v_{p_{2}, M}} v_{p_{3}, M} \overline{v_{p_{4}, M}} v_{p_{5}, M}\right)\right]_{0}^{S}\right. \\
& \left.-i \int_{0}^{S} e^{i(S-\sigma)\left[\partial_{x x}+\Phi_{q, \vec{p}}\right]}\left(\partial_{x x}+\Phi_{q, \vec{p}}\right)^{-1} P_{l o w} \partial_{\sigma}\left\{v_{p_{1}, M} \overline{v_{p_{2}, M}} v_{p_{3}, M} \overline{v_{p_{4}, M}} v_{p_{5}, M}\right\} d \sigma\right\} .
\end{aligned}
$$

We bound the $S$-boundary limit using (2.7) and the boundedness of $\left(\partial_{x x}+\Phi_{q, \vec{p}}\right)^{-1} P_{\text {low }}$ on $L_{x}^{2}(\mathbb{R})$ along with Lemma 8.2 .

$$
\begin{aligned}
& \left\|\sum_{q \in \mathbb{Z}^{2}} \sum_{\vec{p} \in \mathcal{N} \mathcal{R}(q)} e^{-i S\left[|q|^{2}+\Phi_{q, \vec{p}}\right]} e^{i\langle y, q\rangle}\left(\partial_{x x}+\Phi_{q, \vec{p}}\right)^{-1} P_{l o w}\left(v_{p_{1}, M} \overline{v_{p_{2}, M}} v_{p_{3}, M} \overline{v_{p_{4}, M}} v_{p_{5}, M}\right)\right\|_{X^{1}(I)}^{2} \\
& \lesssim \sum_{q \in \mathbb{Z}}\langle q\rangle^{2}\left\|\sum_{\vec{p} \in \mathcal{N} \mathcal{R}(q)} v_{p_{1}, M}(0) \overline{v_{p_{2}, M}}(0) v_{p_{3}, M}(0) \overline{v_{p_{4}, M}}(0) v_{p_{5}, M}(0)\right\|_{H_{x}^{1}(\mathbb{R})}^{2} \\
& +\sum_{q \in \mathbb{Z}}\langle q\rangle^{2}\left\|\left(i \partial_{t}+\partial_{x x}\right) \sum_{\vec{p} \in \mathcal{N} \mathcal{R}(q)} v_{p_{1}, M} \overline{v_{p_{2}, M}} v_{p_{3}, M} \overline{v_{p_{4}, M}} v_{p_{5}, M}\right\|_{L_{t}^{1} H_{x}^{1}}^{2} \\
& \lesssim_{\left\|\vec{v}_{0}\right\|_{h^{5} H^{4}}} M^{4} .
\end{aligned}
$$


The 0-boundary limit is bounded more directly as follows:

$$
\begin{aligned}
& \left\|\sum_{q \in \mathbb{Z}^{2}} \sum_{\vec{p} \in \mathcal{N} \mathcal{R}(q)} e^{i S \Delta_{\mathbb{R} \times \mathbb{T}^{2}}} e^{i\langle y, q\rangle}\left(\partial_{x x}+\Phi_{q, \vec{p}}\right)^{-1} P_{l o w}\left[v_{p_{1}, M} \overline{v_{p_{2}, M}} v_{p_{3}, M} \overline{v_{p_{4}, M}} v_{p_{5}, M}\right](0)\right\|_{X^{1}(I)}^{2} \\
& \lesssim \sum_{q \in \mathbb{Z}^{2}}\langle q\rangle^{2}\left\|\sum_{\vec{p} \in \mathcal{N} \mathcal{R}(q)}\left(v_{p_{1}, M} \overline{v_{p_{2}, M}} v_{p_{3}, M} \overline{v_{p_{4}, M}} v_{p_{5}, M}\right)(0)\right\|_{H_{x}^{1}(\mathbb{R})}^{2} \lesssim_{\left\|\vec{v}_{0}\right\|_{h^{5} H^{4}}} M^{4} .
\end{aligned}
$$

Finally, we also have that

$$
\begin{aligned}
& \| \sum_{q \in \mathbb{Z}^{2}} \sum_{\vec{p} \in \mathcal{N} \mathcal{R}(q)} e^{-i S\left[|q|^{2}+\Phi_{q, \vec{p}}\right]} e^{i\langle y, q\rangle} \\
& \int_{0}^{S} e^{i(S-\sigma)\left[\partial_{x x}+\Phi_{q, \vec{p}}\right]}\left(\partial_{x x}+\Phi_{q, \vec{p}}\right)^{-1} P_{l o w} \partial_{\sigma}\left\{v_{p_{1}, M} \overline{v_{p_{2}, M}} v_{p_{3}, M} \overline{v_{p_{4}, M}} v_{p_{5}, M}\right\} d \sigma \|_{X^{1}}^{2} \\
& \lesssim \sum_{q \in \mathbb{Z}^{2}}\langle q\rangle^{2}\left\|\sum_{\vec{p} \in \mathcal{N} \mathcal{R}(q)} \partial_{\sigma}\left\{v_{p_{1}, M} \overline{v_{p_{2}, M}} v_{p_{3}, M} \overline{v_{p_{4}, M}} v_{p_{5}, M}\right\}\right\|_{L_{t}^{1} H_{x}^{1}}^{2} \lesssim_{\left\|\vec{v}_{0}\right\|_{h^{5} H^{4}}} M^{4} .
\end{aligned}
$$

This finishes the proof of (5.26).

Independently, we also have that

$$
\left\|V_{M}\right\|_{L_{t}^{\infty} H_{x, y}^{1}\left(\mathbb{R} \times \mathbb{T}^{2} \times I\right)}^{2} \leq \sum_{q \in \mathbb{Z}^{2}}\langle q\rangle^{2}\left\|v_{q, M}\right\|_{L_{t}^{\infty} H_{x}^{1}}^{2} \leq 2\|\vec{u}(0)\|_{h^{1} L^{2}}^{2}+M^{2}\|\vec{v}\|_{h^{1} H^{1}}^{2}
$$

and that

$$
\left\|V_{M}\right\|_{X^{1}(I)} \lesssim_{\|\vec{u}\|_{h^{1} L^{2}}} 1+C\left(\|\vec{v}\|_{h^{5} H^{4}}\right) M
$$

Indeed, using Lemma 8.2 in the third line,

$$
\begin{aligned}
\left\|\left(i \partial_{t}+\Delta_{\mathbb{R} \times \mathbb{T}^{2}}\right) V_{M}\right\|_{N^{1}(I)}^{2} & \lesssim \sum_{q \in \mathbb{Z}^{2}}\langle q\rangle^{2}\left\|\sum_{\vec{p} \in \mathcal{R}(q)} v_{p_{1}, M} \overline{v_{p_{2}, M}} v_{p_{3}, M} \overline{v_{p_{4}, M}} v_{p_{5}, M}\right\|_{L_{t}^{1} H_{x}^{1}}^{2} \\
& \lesssim \sum_{q \in \mathbb{Z}^{2}}\left[\sum_{\vec{p} \in \mathcal{R}(q)} \Pi_{k=1}^{5}\left\langle p_{k}\right\rangle\left\|v_{p_{k}, M}\right\|_{L_{t}^{5} W_{x}^{1,10}} \times\langle q\rangle \Pi_{k=1}^{5}\left\langle p_{k}\right\rangle^{-1}\right]^{2} \\
& \lesssim\left[\sum_{p \in \mathbb{Z}^{2}}\langle p\rangle^{2}\left\|v_{p, M}\right\|_{L_{t}^{5} W_{x}^{1,10}}^{2}\right]^{5}
\end{aligned}
$$

and appealing to (5.25), we can justify (5.27).

Using Proposition 4.6, we conclude that, for $M$ small enough (depending on $\vec{v}_{0}$ ), the solution $U_{M}$ of (1.1) with initial data $V_{M}(0)$ exists on $I$ and that

$$
\left\|U_{M}-V_{M}\right\|_{X^{1}(I)} \lesssim \varepsilon_{1}+C\left(\left\|\vec{v}_{0}\right\|_{h^{5} H^{4}}\right) M,
$$

which ends the proof.

To understand linear and nonlinear evolutions beyond the large-scale window we need an additional extinction lemma: 
Lemma 5.8. For any $\psi \in H^{0,1}\left(\mathbb{R} \times \mathbb{T}^{2}\right)$ and any $\varepsilon>0$, there exists $T_{0}=T(\psi, \varepsilon)$ and $M_{0}=M_{0}(\psi, \epsilon)$ such that for any $T \geq T_{0}$ and any $M \leq M_{0}$,

$$
\left\|e^{i t \Delta_{\mathbb{R} \times \mathbb{T}^{2}}} T_{M}^{l s} \psi\right\|_{Z\left(\left\{M^{2}|t| \geq T_{0}\right\}\right)} \lesssim \varepsilon .
$$

Proof. By Strichartz estimates on $\mathbb{R}$, and dominated convergence, there exists $T_{0}=T_{0}(\psi, \varepsilon)$ such that

$$
\sum_{p \in \mathbb{Z}^{2}}\langle p\rangle^{2}\left\|e^{i t \partial_{x x}} \psi_{p}\right\|_{L_{x, t}^{6}\left(\mathbb{R} \times\left\{|t| \geq T_{0}\right\}\right)}^{2} \leq \varepsilon^{1000} .
$$

Let $I=\left\{|t| \geq T_{0}\right\}$ and $I_{M}=\left\{M^{2}|t| \geq T_{0}\right\}$. We have that

$$
\begin{aligned}
e^{i t \Delta_{\mathbb{R} \times \mathbb{T}^{2}}} T_{M}^{l s} \psi & =\sum_{q \in \mathbb{Z}^{2}} e^{i\left(\langle q, y\rangle-t|q|^{2}\right)}\left(M^{1 / 2} e^{i M^{2} t \partial_{x x}} \tilde{\psi}_{q}^{M}(M x)\right), \quad \tilde{\psi}^{M}=P_{\leq M^{-1 / 100}}^{x} \psi \\
& =\sum_{q \in \mathbb{Z}^{2}} e^{i\left(\langle q, y\rangle-|q|^{2} t\right)} v_{q, M}(t, x)
\end{aligned}
$$

where we denoted by:

$$
v_{p, M}(x, t)=M^{1 / 2} e^{i M^{2} t \partial_{x x}} \tilde{\psi}_{p}^{M}(M x) .
$$

Using the fact that $e^{i\langle q, y\rangle} v_{p, M}(x, t)$ is supported in Fourier space in the box centered at $q$ of radius 2 and Bernstein's estimate in $y$, we can estimate on $\mathbb{R} \times \mathbb{T}^{2} \times I_{M}$ :

$$
\left\|P_{N} e^{i t \Delta_{\mathbb{R} \times \mathbb{T}^{2}}} T_{M}^{l s} \psi\right\|_{L_{x, y, t}^{6}} \lesssim N^{2 / 3}\left\|\left(\sum_{|q| \sim N}\left|v_{q, M}(x, t)\right|^{2}\right)^{1 / 2}\right\|_{L_{x, t}^{6}} \lesssim N^{2 / 3}\left(\sum_{|q| \sim N}\left\|v_{q, M}\right\|_{L_{x, t}^{6}\left(\mathbb{R}_{x} \times \mathbb{R}_{t}\right)}^{2}\right)^{1 / 2} .
$$

Consequently we see that

$$
\begin{aligned}
\sum_{N \geq 1} N^{\frac{2}{3}} \| P_{N} e^{i t \Delta_{\mathbb{R} \times \mathbb{T}^{2}} T_{M}^{l s} \psi \|_{L_{x, y, t}^{6}}^{2}\left(\mathbb{R} \times \mathbb{T}^{2} \times I_{M}\right)} & \lesssim \sum_{N \geq 1} N^{2} \sum_{|q| \sim N}\left\|v_{q, M}\right\|_{L_{x, t}^{6}\left(\mathbb{R} \times I_{M}\right)}^{2} \\
& \lesssim \sum_{q \in \mathbb{Z}^{2}}\langle q\rangle^{2}\left\|e^{i t \partial_{x x}} \widetilde{\psi}_{q}^{M}\right\|_{L_{x, t}^{6}(\mathbb{R} \times I)}^{2} \lesssim \varepsilon^{1000},
\end{aligned}
$$

if $M$ is chosen large enough depending on $\psi$ and $\varepsilon$. On the other hand, by Strichartz estimates (3.2), we also have that

$$
\sum_{N \geq 1} N^{5-\frac{p_{0}}{2}}\left\|P_{N} e^{i t \Delta_{\mathbb{R} \times \mathbb{T}^{2}}} T_{M}^{l s} \psi\right\|_{l_{\gamma}^{\frac{4 p_{0}}{p_{0}-2}} L_{x, y, t}^{p_{0}}}^{p_{0}} \lesssim\left\|T_{M}^{l s} \psi\right\|_{H^{1}\left(\mathbb{R} \times \mathbb{T}^{2}\right)}^{p_{0}} \lesssim\|\psi\|_{H^{0,1}\left(\mathbb{R} \times \mathbb{T}^{2}\right)}^{p_{0}}
$$

for $p_{0}=4.1,1000$. Interpolating this with (5.30) gives (5.28).

We are now ready to describe the nonlinear solutions of (1.1) corresponding to large-scale profiles. In view of the profile analysis in the next section, we need to consider renormalized large-scale frames defined by:

$$
\begin{aligned}
\widetilde{\mathcal{F}_{l s}}:= & \left\{\left(M_{k}, t_{k}, p_{k}, \xi_{k}\right)_{k}: M_{k} \leq 1, M_{k} \rightarrow 0, t_{k} \in \mathbb{R}, p_{k}=\left(x_{k}, 0\right) \in \mathbb{R} \times \mathbb{T}^{2} \text { and } \xi_{k} \in \mathbb{R}, \xi_{k} \rightarrow \xi_{\infty} \in \mathbb{R}\right. \\
& \text { and such that either } \left.t_{k} \equiv 0 \text { or } M_{k}^{2} t_{k} \rightarrow \pm \infty, \text { and either } \xi_{k} \equiv 0 \text { or } M_{k}^{-1} \xi_{k} \rightarrow \pm \infty\right\}
\end{aligned}
$$

We can then describe the solution of (1.1) with initial data given by a large-scale profile as follows: 
Proposition 5.9. Assume that Conjecture 5.5 holds true. Fix a renormalized large-scale frame, $\mathcal{S}=$ $\left(M_{k}, t_{k},\left(x_{k}, 0\right), \xi_{k}\right)_{k} \in \widetilde{\mathcal{F}}_{l s}, \psi \in H^{0,1}\left(\mathbb{R} \times \mathbb{T}^{2}\right)$, and let

$$
U_{k}(0)=\Pi_{t_{k}, x_{k}} e^{i \xi_{k} x} T_{M_{k}}^{l s} \psi
$$

(i) For $k$ large enough (depending only on $\psi, \mathcal{S}$ ), there is a nonlinear solution $U_{k} \in X_{c}^{1}(\mathbb{R})$ of the equation (1.1) satisfying:

$$
\left\|U_{k}\right\|_{X^{1}(\mathbb{R})} \lesssim_{E_{l s}(\psi)} 1 .
$$

(ii) There exists a solution $\vec{v} \in C\left(\mathbb{R}: h^{1} L^{2}\right)$ of (1.6) with scattering data $\vec{v}_{0}{ }^{ \pm \infty}$ such that the following holds, up to a subsequence: for any $\varepsilon>0$, there exists $T(\psi, \varepsilon)$ such that for all $T \geq T(\psi, \varepsilon)$, there holds that

$$
\left\|U_{k}-W_{k}\right\|_{X^{1}\left(\left\{\left|t-t_{k}\right| \leq T M_{k}^{-2}\right\}\right)} \leq \varepsilon
$$

for $k$ large enough, where

$$
W_{k}(x, t)=e^{-i \tau\left|\xi_{k}\right|^{2}} e^{i x \xi_{k}} V_{M_{k}}\left(x-x_{k}-2 \xi_{k} \tau, y, \tau\right), \quad \tau=t-t_{k}
$$

with $V_{M_{k}}$ defined in terms of $\vec{v}$ as in (5.23). In addition, up to a subsequence,

$$
\left\|U_{k}(t)-\Pi_{t_{k}-t, x_{k}} e^{i x \xi_{k}} T_{M_{k}}^{l^{s}}{\underline{\overrightarrow{v_{0}}}}^{ \pm \infty}\right\|_{X^{1}\left(\left\{ \pm\left(t-t_{k}\right) \geq T M_{k}^{-2}\right\}\right)} \leq \varepsilon,
$$

for $k$ large enough (depending on $\psi, \varepsilon, T$ ).

Proof. Without loss of generality we may assume that $x_{k}=0$. Using a Galilean transform and the fact that $\xi_{k}$ is bounded, we may assume that $\xi_{k}=0$ for all $k$. We first consider the case when $t_{k}=0$ for all $k$ and we let $\vec{v}$ be the solution of (1.6) with initial data $\vec{\psi}$. Then, by Proposition [5.6, we see that there exists $T_{0}=T_{0}(\psi, \varepsilon)$ such that

$$
\begin{array}{r}
\sup _{t \geq T_{0}}\left\|\vec{u}(t)-e^{i t \partial_{x x}} \vec{v}_{0}^{+\infty}\right\|_{h^{1} L^{2}}+\left\|e^{i t \partial_{x x}} \vec{v}_{0}^{+\infty}\right\|_{\vec{W}\left(\mathbb{R} \times\left\{t \geq T_{0}\right\}\right)} \leq \varepsilon, \\
\sup _{t \leq-T_{0}}\left\|\vec{u}(t)-e^{i t \partial_{x x}} \vec{v}_{0}^{-\infty}\right\|_{h^{1} L^{2}}+\left\|e^{i t \partial_{x x}} \vec{v}_{0}^{-\infty}\right\|_{\vec{W}\left(\mathbb{R} \times\left\{t \leq-T_{0}\right\}\right)} \leq \varepsilon,
\end{array}
$$

Fix $T \geq T_{0}$. Applying Lemma 5.7, we see that, provided that $k$ is large enough,

$$
\left\|U_{k}-V_{M_{k}}\right\|_{\tilde{X}^{1}\left(\left\{|t| \leq T M_{k}^{-2}\right\}\right)} \leq \varepsilon .
$$

This and Strichartz estimates, together with (5.35), Lemma 5.8 imply that

$$
\left\|e^{i t \Delta_{\mathbb{R} \times \mathbb{T}^{2}}} U_{k}\left( \pm T M_{k}^{-2}\right)\right\|_{Z\left( \pm t \geq M_{k}^{-2} T\right)} \leq \varepsilon .
$$

At this point, Proposition 4.4 shows that $U_{k}$ extends to a global solution $U_{k} \in X_{c}^{1}(\mathbb{R})$ satisfying (5.34).

We now turn to the case when $M_{k}^{-2}\left|t_{k}\right| \rightarrow \infty$. For definiteness, we assume that $M_{k}^{-2} t_{k} \rightarrow+\infty$ and let $\vec{v}$ be the solutions to (1.6) satisfying:

$$
\left\|\vec{v}(t)-e^{i t \partial_{x x}} \vec{\psi}\right\|_{h^{1} L^{2}} \rightarrow 0
$$

as $t \rightarrow-\infty$. Let $\psi^{\prime}=\underline{\vec{v}}(0) \in H^{0,1}\left(\mathbb{R} \times \mathbb{T}^{2}\right)$ and let $V_{k}$ be the solution of (1.1) with initial data $T_{M_{k}}^{l s} \psi^{\prime}$. Applying the first case of the proof to the frame $\left(N_{k}, 0,0,0\right)$ and the family $V_{k}$ we conclude that:

$$
\left\|V_{k}\left(-t_{k}\right)-\Pi_{t_{k}, 0} T_{M_{k}}^{l s} \psi\right\|_{H^{1}\left(\mathbb{R} \times \mathbb{T}^{2}\right)} \rightarrow 0
$$

as $k \rightarrow \infty$. The conclusion of the proof now follows from Proposition 4.6 and by inspecting the behavior of $V_{k}$. 


\section{Profile Decomposition}

Recall the definitions (5.11), (5.12) and (5.17). We consider three different kind of profiles corresponding to different frames.

Definition 6.1 (Frames and Profiles). (1) We define a frame to be sequence $\left(N_{k}, t_{k}, p_{k}, \xi_{k}\right)_{k}$ in $2^{\mathbb{Z}} \times$ $\mathbb{R} \times\left(\mathbb{R} \times \mathbb{T}^{2}\right) \times \mathbb{R}$. We distinguish the following three different types of frames:

(a) A Euclidean frame is a sequence $\mathcal{F}_{e}=\left(N_{k}, t_{k}, p_{k}, 0\right)_{k}$ with $N_{k} \geq 1, N_{k} \rightarrow+\infty, t_{k} \in \mathbb{R}$, $p_{k} \in \mathbb{R} \times \mathbb{T}^{2}$.

(b) A large-scale frame is a sequence $\mathcal{F}_{l s}=\left(M_{k}, t_{k}, p_{k}, \xi_{k}\right)_{k}$ with $M_{k} \leq 1, M_{k} \rightarrow 0, t_{k} \in \mathbb{R}$, $p_{k}=\left(x_{k}, 0\right) \in \mathbb{R} \times \mathbb{T}^{2}$ and $\xi_{k} \in \mathbb{R}, \xi_{k} \rightarrow \xi_{\infty} \in \mathbb{R}$.

(c) A Scale-1 frame is a sequence $\mathcal{F}_{1}=\left(1, t_{k}, p_{k}, 0\right)_{k}$ with $t_{k} \in \mathbb{R}, p_{k} \in \mathbb{R} \times \mathbb{T}^{2}$.

(2) We say that two frames $\left(N_{k}, t_{k}, p_{k}, \xi_{k}\right)_{k}$ and $\left(M_{k}, s_{k}, q_{k}, \eta_{k}\right)_{k}$ are orthogonal if

$\lim _{k \rightarrow+\infty}\left(\left|\ln \frac{N_{k}}{M_{k}}\right|+N_{k}^{2}\left|t_{k}-s_{k}\right|+N_{k}^{-1}\left|\xi_{k}-\eta_{k}\right|+N_{k}\left|\left(p_{k}-q_{k}\right)-2\left(t_{k}-s_{k}\right) \xi_{k}\right|\right)=+\infty$.

Two frames that are not orthogonal are called equivalent.

(3) Associated to each of the above three types of frames, we associate a profile defined as:

(a) If $\mathcal{O}=\left(N_{k}, t_{k}, p_{k}, 0\right)_{k}$ is a Euclidean frame and if $\phi \in \dot{H}^{1}\left(\mathbb{R}^{3}\right)$, we define the Euclidean profile associated to $(\phi, \mathcal{O})$ as the sequence $\widetilde{\phi}_{\mathcal{O}, k}$ with

$$
\widetilde{\phi}_{\mathcal{O}, k}(x, y):=\Pi_{t_{k}, p_{k}}\left(T_{N_{k}}^{e} \phi\right)(x, y) .
$$

(b) If $\mathcal{O}=\left(M_{k}, t_{k}, p_{k}, \xi_{k}\right)_{k}$ is a large scale frame, if $p_{k}=\left(x_{k}, 0\right)$ and if $\psi \in H^{0,1}\left(\mathbb{R} \times \mathbb{T}^{2}\right)$, we define the large scale profile associated to $(\psi, \mathcal{O})$ as the sequence $\widetilde{\psi}_{\mathcal{O}, k}$ with

$$
\widetilde{\psi}_{\mathcal{O}, k}(x, y):=\Pi_{t_{k}, p_{k}}\left[e^{i \xi_{k} x} T_{M_{k}}^{l s} \psi(x, y)\right] .
$$

(c) If $\mathcal{O}=\left(1, t_{k}, p_{k}, 0\right)$ is a scale-1 frame and $W \in H^{1}\left(\mathbb{R} \times \mathbb{T}^{2}\right)$, we define the Scale-1 profile associated to $(W, \mathcal{O})$ as $\widetilde{W}_{\mathcal{O}, k}$ with

$$
\widetilde{W}_{\mathcal{O}, k}=\Pi_{t_{k}, p_{k}} W
$$

(4) Finally, we say that a sequence of functions $\left\{f_{k}\right\}_{k} \subset H^{1}\left(\mathbb{R} \times \mathbb{T}^{2}\right)$ is absent from a frame $\mathcal{O}$ if, up to a subsequence:

$$
\left\langle f_{k}, \widetilde{\psi}_{\mathcal{O}, k}\right\rangle_{H^{1} \times H^{1}} \rightarrow 0
$$

as $k \rightarrow+\infty$ for any profile $\widetilde{\psi}_{\mathcal{O}, k}$ associated with $\mathcal{O}$.

6.1. Orthogonality of profiles. This section summarizes some orthogonality and equivalence properties of frames. These are included in the following lemma:

Lemma 6.2 (Frame equivalences and orthogonality). $\quad$ (1) Suppose that $\mathcal{O}$ and $\mathcal{O}^{\prime}$ are equivalent Euclidean (respectively large-scale, or scale-1) frames, then there exists an isometry $L$ of $\dot{H}^{1}\left(\mathbb{R}^{3}\right)$ (resp. $\quad H^{0,1}\left(\mathbb{R} \times \mathbb{T}^{2}\right), H^{1}\left(\mathbb{R} \times \mathbb{T}^{2}\right)$ ) such that, for any profile generator $\psi \in \dot{H}^{1}\left(\mathbb{R}^{3}\right)$ (resp. $\left.H^{0,1}\left(\mathbb{R} \times \mathbb{T}^{2}\right), H^{1}\left(\mathbb{R} \times \mathbb{T}^{2}\right)\right)$ it holds that, up to a subsequence:

$$
\limsup _{k \rightarrow+\infty}\left\|\widetilde{L \psi}_{\mathcal{O}, k}-\widetilde{\psi}_{\mathcal{O}^{\prime}, k}\right\|_{H^{1}\left(\mathbb{R} \times \mathbb{T}^{2}\right)}=0
$$


(2) Suppose that $\mathcal{O}$ and $\mathcal{O}^{\prime}$ are orthogonal frames and $\widetilde{\psi}_{\mathcal{O}, k}$ and $\widetilde{\phi}_{\mathcal{O}^{\prime}, k}$ are two profiles associated with $\mathcal{O}$ and $\mathcal{O}^{\prime}$ respectively. Then

$$
\begin{array}{r}
\lim _{k \rightarrow+\infty}\left\langle\widetilde{\psi}_{\mathcal{O}, k}, \widetilde{\phi}_{\mathcal{O}^{\prime}, k}\right\rangle_{H^{1} \times H^{1}\left(\mathbb{R} \times \mathbb{T}^{2}\right)}=0, \\
\lim _{k \rightarrow+\infty}\left\langle\left|\widetilde{\psi}_{\mathcal{O}, k}\right|^{3},\left|\widetilde{\phi}_{\mathcal{O}^{\prime}, k}\right|^{3}\right\rangle=0 .
\end{array}
$$

(3) If $\mathcal{O}$ is a Euclidean frame and $\widetilde{\psi}_{\mathcal{O}, k}, \widetilde{\phi}_{\mathcal{O}, k}$ are two profiles associated to $\mathcal{O}$, then:

$$
\begin{aligned}
\lim _{k \rightarrow+\infty}\left\|\widetilde{\psi}_{\mathcal{O}, k}\right\|_{L^{2}}+\left\|\widetilde{\phi}_{\mathcal{O}, k}\right\|_{L^{2}} & =0, \\
\lim _{k \rightarrow+\infty}\left\langle\widetilde{\psi}_{\mathcal{O}, k}, \widetilde{\phi}_{\mathcal{O}, k}\right\rangle_{H^{1} \times H^{1}\left(\mathbb{R} \times \mathbb{T}^{2}\right)} & =\langle\psi, \phi\rangle_{\dot{H}^{1} \times \dot{H}^{1}\left(\mathbb{R}^{3}\right)} .
\end{aligned}
$$

(4) If $\mathcal{O}$ is a scale- 1 frame and $\widetilde{\psi}_{\mathcal{O}, k}, \widetilde{\phi}_{\mathcal{O}, k}$ are two profiles associated to $\mathcal{O}$, then:

$$
\lim _{k \rightarrow+\infty}\left\langle\widetilde{\psi}_{\mathcal{O}, k}, \widetilde{\phi}_{\mathcal{O}, k}\right\rangle_{H^{1} \times H^{1}\left(\mathbb{R} \times \mathbb{T}^{2}\right)}=\langle\psi, \phi\rangle_{H^{1} \times H^{1}\left(\mathbb{R} \times \mathbb{T}^{2}\right)}
$$

(5) If $\mathcal{O}$ is a large-scale frame and $\widetilde{\psi}_{\mathcal{O}, k}, \widetilde{\phi}_{\mathcal{O}, k}$ are two profiles associated to $\mathcal{O}$, then:

$$
\begin{gathered}
\lim _{k \rightarrow+\infty}\left\|\widetilde{\psi}_{\mathcal{O}, k}\right\|_{L_{x, y}^{6}\left(\mathbb{R} \times \mathbb{T}^{2}\right)}=0, \\
\lim _{k \rightarrow+\infty}\left\langle\widetilde{\psi}_{\mathcal{O}, k}, \widetilde{\phi}_{\mathcal{O}, k}\right\rangle_{H^{1} \times H^{1}\left(\mathbb{R} \times \mathbb{T}^{2}\right)}=\langle\psi, \phi\rangle_{H^{0,1} \times H^{0,1}\left(\mathbb{R} \times \mathbb{T}^{2}\right)}+\left|\xi_{\infty}\right|^{2}\langle\psi, \phi\rangle_{L^{2} \times L^{2}\left(\mathbb{R} \times \mathbb{T}^{2}\right)} \simeq\langle\psi, \phi\rangle_{H^{0,1} \times H^{0,1}\left(\mathbb{R} \times \mathbb{T}^{2}\right)} \\
\text { where here } \simeq \text { means bounded above and below by } C \text { and } C^{-1} \text { respectively for some } C \geq 1 .
\end{gathered}
$$

Proof. The proof is tedious but straightforward, so we omit the details (cf. [33], 34]).

6.2. Profile Decomposition. We are now in a position to perform a profile decomposition for bounded sequences of functions in $H^{1}\left(\mathbb{R} \times \mathbb{T}^{2}\right)$.

Proposition 6.3. Assume that $\phi_{k}$ is a sequence satisfying

$$
\sup _{k \geq 0}\left\|\phi_{k}\right\|_{L^{2}\left(\mathbb{R} \times \mathbb{T}^{2}\right)}+\left\|\nabla_{x, y} \phi_{k}\right\|_{L^{2}\left(\mathbb{R} \times \mathbb{T}^{2}\right)} \leq E<+\infty,
$$

then there exists a subsequence (which we also denote by $\phi_{k}$ ), a family of Euclidean profiles $\widetilde{\varphi}_{\mathcal{O}^{\alpha}, k}^{\alpha}$, a family of large scale profiles $\widetilde{\psi}_{\mathcal{S}^{\beta}, k}^{\beta}$, a family of scale 1 profiles $\widetilde{W}_{\mathcal{O}^{\gamma}, k}^{\gamma}$ such that, for any $A \geq 1$ and any $k \geq 0$ in the subsequence

$$
\phi_{k}(x, y)=\sum_{1 \leq \alpha \leq A} \widetilde{\varphi}_{\mathcal{O}^{\alpha}, k}^{\alpha}(x, y)+\sum_{1 \leq \beta \leq A} \widetilde{\psi}_{\mathcal{S}^{\beta}, k}^{\beta}(x, y)+\sum_{1 \leq \gamma \leq A} \widetilde{W}_{\mathcal{O}^{\gamma}, k}^{\gamma}(x, y)+R_{k}^{A}(x, y)
$$

with

$$
\lim _{A \rightarrow+\infty} \limsup _{k \rightarrow+\infty}\left\|e^{i t \Delta_{\mathbb{R} \times \mathbb{T}^{2}}} R_{k}^{A}\right\|_{Z(\mathbb{R})}=0
$$


In addition, all the frames are pairwise orthogonal and we have the following orthogonality property:

$$
\begin{aligned}
M\left(\phi_{k}\right) & =\sum_{1 \leq \beta \leq A} M\left(\psi^{\beta}\right)+\sum_{1 \leq \gamma \leq A} M\left(W^{\gamma}\right)+M\left(R_{k}^{A}\right)+o_{A, k \rightarrow+\infty}(1), \\
\left\|\nabla_{x, y} \phi_{k}\right\|_{L^{2}\left(\mathbb{R} \times \mathbb{T}^{2}\right)}^{2} & =\sum_{1 \leq \alpha \leq A}\left\|\varphi^{\alpha}\right\|_{\dot{H}^{1}\left(\mathbb{R}^{3}\right)}^{2}+\sum_{1 \leq \beta \leq A}\left[\left|\xi_{\infty}^{\beta}\right|^{2} M\left(\psi^{\beta}\right)+\left\|\nabla_{y} \psi^{\beta}\right\|_{L^{2}\left(\mathbb{R} \times \mathbb{T}^{2}\right)}^{2}\right] \\
& +\sum_{1 \leq \gamma \leq A}\left\|\nabla_{x, y} W^{\gamma}\right\|_{L^{2}\left(\mathbb{R} \times \mathbb{T}^{2}\right)}^{2}+\left\|\nabla_{x, y} R_{k}^{A}\right\|_{L^{2}\left(\mathbb{R} \times \mathbb{T}^{2}\right)}^{2}+o_{A, k \rightarrow+\infty}(1), \\
\left\|\phi_{k}\right\|_{L^{6}\left(\mathbb{R} \times \mathbb{T}^{2}\right)}^{6} & =\sum_{1 \leq \alpha \leq A}\left\|\varphi^{\alpha}\right\|_{L^{6}\left(\mathbb{R}^{3}\right)}^{6}+\sum_{1 \leq \gamma \leq A}\left\|W^{\gamma}\right\|_{L^{6}\left(\mathbb{R} \times \mathbb{T}^{2}\right)}^{6}+o_{A \rightarrow+\infty, k \rightarrow+\infty}(1),
\end{aligned}
$$

where $\xi_{\infty}^{\beta}=\lim _{k \rightarrow+\infty} \xi_{k}^{\beta}, o_{A, k \rightarrow+\infty}(1) \rightarrow 0$ as $k \rightarrow+\infty$ for each fixed $A$, and $o_{A \rightarrow+\infty, k \rightarrow+\infty}(1) \rightarrow 0$ in the ordered limit $\lim _{A \rightarrow+\infty} \lim _{k \rightarrow+\infty}$.

As in [34], this follows from iteration of the following finitary statement:

Lemma 6.4. Let $\delta>0$. Assume that $\phi_{k}$ is a sequence satisfying

$$
\sup _{k \geq 0}\left\|\phi_{k}\right\|_{L^{2}\left(\mathbb{R} \times \mathbb{T}^{2}\right)}+\left\|\nabla_{x, y} \phi_{k}\right\|_{L^{2}\left(\mathbb{R} \times \mathbb{T}^{2}\right)} \leq E<+\infty,
$$

then there exists a subsequence (which we also denote by $\phi_{k}$ ), $A=A(E, \delta)$ Euclidean profiles $\widetilde{\varphi}_{\mathcal{O}^{\alpha}, k}^{\alpha}, A$ large scale profiles $\widetilde{\psi}_{\mathcal{S}^{\beta}, k}^{\beta}$, and $A$ scale 1 profiles $\widetilde{W}_{\mathcal{O}^{\gamma}, k}^{\gamma}$ such that, for any $k \geq 0$ in the subsequence

$$
\phi_{k}(x, y)=\sum_{1 \leq \alpha \leq A} \widetilde{\varphi}_{\mathcal{O}^{\alpha}, k}^{\alpha}(x, y)+\sum_{1 \leq \beta \leq A} \widetilde{\psi}_{\mathcal{S}^{\beta}, k}^{\beta}(x, y)+\sum_{1 \leq \gamma \leq A} \widetilde{W}_{\mathcal{O}^{\gamma}, k}^{\gamma}(x, y)+R_{k}^{A}(x, y)
$$

with

$$
\limsup _{k \rightarrow+\infty}\left[\left\|e^{i t \Delta_{\mathbb{R} \times \mathbb{T}^{2}}} R_{k}^{A}\right\|_{Z(\mathbb{R})}+\sup _{t}\left\|e^{i t \Delta_{\mathbb{R} \times \mathbb{T}^{2}}} R_{k}^{A}\right\|_{L_{x, y}^{6}\left(\mathbb{R} \times \mathbb{T}^{2}\right)}\right] \leq \delta .
$$

In addition, all the frames are pairwise orthogonal and we have the following orthogonality property:

$$
\begin{aligned}
M\left(\phi_{k}\right) & =\sum_{1 \leq \beta \leq A} M\left(\psi^{\beta}\right)+\sum_{1 \leq \gamma \leq A} M\left(W^{\gamma}\right)+M\left(R_{k}^{A}\right)+o_{k \rightarrow+\infty}(1), \\
\left\|\nabla_{x, y} \phi_{k}\right\|_{L^{2}\left(\mathbb{R} \times \mathbb{T}^{2}\right)}^{2} & =\sum_{1 \leq \alpha \leq A}\left\|\varphi^{\alpha}\right\|_{\dot{H}^{1}\left(\mathbb{R}^{3}\right)}^{2}+\sum_{1 \leq \beta \leq A}\left[\left|\xi_{\infty}^{\beta}\right|^{2} M\left(\psi^{\beta}\right)+\left\|\nabla_{y} \psi^{\beta}\right\|_{L^{2}\left(\mathbb{R} \times \mathbb{T}^{2}\right)}^{2}\right] \\
& +\sum_{1 \leq \gamma \leq A}\left\|\nabla_{x, y} W^{\gamma}\right\|_{L^{2}\left(\mathbb{R} \times \mathbb{T}^{2}\right)}^{2}+\left\|\nabla_{x, y} R_{k}^{A}\right\|_{L^{2}\left(\mathbb{R} \times \mathbb{T}^{2}\right)}^{2}+o_{k \rightarrow+\infty}(1), \\
\left\|\phi_{k}\right\|_{L^{6}\left(\mathbb{R} \times \mathbb{T}^{2}\right)}^{6} & =\sum_{1 \leq \alpha \leq A}\left\|\varphi^{\alpha}\right\|_{L^{6}\left(\mathbb{R}^{3}\right)}^{6}+\sum_{1 \leq \gamma \leq A}\left\|W^{\gamma}\right\|_{L^{6}\left(\mathbb{R} \times \mathbb{T}^{2}\right)}^{6} \\
& +\left\|R_{k}^{A}\right\|_{L^{6}\left(\mathbb{R} \times \mathbb{T}^{2}\right)}^{6}+o_{k \rightarrow+\infty}(1),
\end{aligned}
$$

where $o_{k \rightarrow+\infty}(1) \rightarrow 0$ as $k \rightarrow+\infty$.

The proof of Lemma 6.4 will be completed in two steps: first we extract the Euclidean and Scale-1 profiles by studying the defects of compactness of a Besov-type Strichartz/Sobolev estimate (cf (6.8)). This extraction leaves only sequences whose linear flow has small critical Besov norm (cf. (6.8), (6.10) but large $Z(\mathbb{R})$ norm, from which we extract the large-scale profiles and finish the proof. 
We start with the first step: For a sequence of functions $\left\{f_{k}\right\}$ in $H^{1}\left(\mathbb{R} \times \mathbb{T}^{2}\right)$, we consider the following functional

$$
\Lambda_{\infty}\left(\left\{f_{k}\right\}\right)=\limsup _{k \rightarrow+\infty}\left\|e^{i t \Delta} f_{k}\right\|_{L_{t}^{\infty} B_{\infty, \infty}^{-\frac{1}{2}}}:=\limsup _{k \rightarrow+\infty} \sup _{(N, t, x, y)} N^{-\frac{1}{2}}\left|\left(e^{i t \Delta} P_{N} f_{k}\right)(x, y)\right|,
$$

where the supremum is taken over all scales $N \geq 1$, times $t \in \mathbb{R}$ and locations $(x, y) \in \mathbb{R} \times \mathbb{T}^{2}$.

Lemma 6.5 (Extraction of the Euclidean and Scale-1 profiles). Let $\nu>0$. Assume that $\phi_{k}$ is a sequence satisfying (6.6), then there exists a subsequence of $\phi_{k}, A=O\left(\nu^{-2}\right)$ Euclidean profiles $\widetilde{\varphi}_{\mathcal{O}^{\alpha}, k}^{\alpha}$, and $A$ scale 1 profiles $\widetilde{W}_{\mathcal{O}^{\gamma}, k}$ such that, for any $k \geq 0$ in the subsequence

$$
\phi_{k}^{\prime}(x, y)=\phi_{k}(x, y)-\sum_{1 \leq \alpha \leq A} \widetilde{\varphi}_{\mathcal{O}^{\alpha}, k}^{\alpha}(x, y)-\sum_{1 \leq \gamma \leq A} \widetilde{W}_{\mathcal{O}^{\gamma}, k}^{\gamma}(x, y)
$$

satisfies

$$
\Lambda_{\infty}\left(\left\{\phi_{k}^{\prime}\right\}\right)<\nu
$$

Besides, all the frames involved are pairwise orthogonal and $\phi_{k}^{\prime}$ is absent from all these frames.

Proof of Lemma 6.5. We first claim that if $\Lambda_{\infty}\left(\left\{f_{k}\right\}\right) \geq \nu$, then there exists a frame $\mathcal{O}$ and an associated (Euclidean or Scale-1) profile $\widetilde{\psi}_{\mathcal{O}_{k}}$ satisfying

$$
\limsup _{k \rightarrow+\infty}\left\|\widetilde{\psi}_{\mathcal{O}, k}\right\|_{H^{1}} \lesssim 1
$$

and

$$
\limsup _{k \rightarrow+\infty}\left|\left\langle f_{k}, \widetilde{\psi}_{\mathcal{O}, k}\right\rangle_{H^{1} \times H^{1}}\right| \gtrsim \nu
$$

In addition, if $f_{k}$ was absent from a family of frames $\mathcal{O}^{\alpha}$, then $\mathcal{O}$ is orthogonal to all the previous frames $\mathcal{O}^{\alpha}$.

Let us prove the claim above. By assumption, up to extracting a subsequence, there exists a sequence $\left(N_{k}, t_{k},\left(x_{k}, y_{k}\right)\right)_{k}$ such that, for all $k$

$$
\begin{aligned}
\frac{\nu}{2} & \leq N_{k}^{-\frac{1}{2}}\left|\left(e^{i t_{k} \Delta} P_{N_{k}} f_{k}\right)\left(x_{k}, y_{k}\right)\right|=\left|\left\langle N_{k}^{-\frac{1}{2}} e^{i t_{k} \Delta} P_{N_{k}} f_{k}, \delta_{\left(x_{k}, y_{k}\right)}\right\rangle_{\mathcal{D} \times \mathcal{D}^{\prime}}\right| \\
& \leq\left|\left\langle f_{k}, N_{k}^{-\frac{1}{2}} e^{-i t_{k} \Delta} P_{N_{k}} \delta_{\left(x_{k}, y_{k}\right)}\right\rangle_{H^{1} \times H^{-1}}\right| .
\end{aligned}
$$

Now, first assume that $N_{k}$ remains bounded, then, up to a subsequence, one may assume that $N_{k} \rightarrow N_{\infty}$ and since $N_{k}$ is dyadic, we may even assume that $N_{k}=N_{\infty}$ for all $k$. In this case, we define the scale-1 profile $\mathcal{O}=\left(1, t_{k},\left(x_{k}, y_{k}\right), 0\right)_{k}$ and

$$
\psi=(1-\Delta)^{-1} N_{\infty}^{-\frac{1}{2}} P_{N_{\infty}} \delta_{(0,0)} .
$$

Inequalities (6.12) is direct. The fact that $\psi$ is bounded in $H^{1}$ uniformly in $N_{\infty}$ follows from the limit below and implies (6.11).

Now, we assume that $N_{k} \rightarrow+\infty$ and we define the Euclidean frame $\mathcal{O}=\left(N_{k}, t_{k},\left(x_{k}, y_{k}\right), 0\right)_{k}$ and the function

$$
\psi=\mathcal{F}_{\mathbb{R}^{3}}^{-1}\left(|\zeta|^{-2}\left[\eta^{3}(\zeta)-\eta^{3}(2 \zeta)\right]\right) \in H^{1}\left(\mathbb{R}^{3}\right), \quad \zeta=\left(\zeta_{1}, \zeta_{2}, \zeta_{3}\right) \in \mathbb{R}^{3} .
$$

It is not hard to prove that

$$
\begin{aligned}
& \lim _{k \rightarrow+\infty}\left\|\left(1-\Delta_{\mathbb{R} \times \mathbb{T}^{2}}\right) T_{N_{k}} \psi-N_{k}^{-\frac{1}{2}} P_{N_{k}} \delta_{(0,0)}\right\|_{L^{6 / 5}\left(\mathbb{R} \times \mathbb{T}^{2}\right)}=0 \\
& \limsup _{k \rightarrow+\infty}\left\|T_{N_{k}} \psi\right\|_{H^{1}\left(\mathbb{R} \times \mathbb{T}^{2}\right)} \lesssim 1 .
\end{aligned}
$$


Thus $\left\|\left(1-\Delta_{\mathbb{R} \times \mathbb{T}^{2}}\right) T_{N_{k}} \psi-N_{k}^{-\frac{1}{2}} P_{N_{k}} \delta_{(0,0)}\right\|_{H^{-1}\left(\mathbb{R} \times \mathbb{T}^{2}\right)} \rightarrow 0$ and we conclude that

$$
\frac{\nu}{2} \lesssim\left|\left\langle f_{k}, N_{k}^{-\frac{1}{2}} e^{-i t_{k} \Delta_{\mathbb{R} \times \mathbb{T}^{2}}} P_{N_{k}} \delta_{\left(x_{k}, y_{k}\right)}\right\rangle_{H^{1} \times H^{-1}}\right| \lesssim\left|\left\langle f_{k},\left(1-\Delta_{\mathbb{R} \times \mathbb{T}^{2}}\right) \widetilde{\psi}_{\mathcal{O}, k}\right\rangle_{H^{1} \times H^{-1}}\right| .
$$

This gives (6.12).

The last claim about orthogonality of $\mathcal{O}$ with $\mathcal{O}^{\alpha}$ follows from Lemma 6.2 and the existence of a nonzero scalar product in (6.12).

Now, continuing with the sequence $\left\{f_{k}\right\}_{k}$ as above, if the frame selected in the previous step was a Scale-1 frame, $\mathcal{O}=\left(1, t_{k}, p_{k}, 0\right)_{k}$, we consider the sequence

$$
g_{k}(x, y):=e^{i t_{k} \Delta} f_{k}\left((x, y)+p_{k}\right)=\Pi_{-\left(t_{k}, p_{k}\right)} f_{k} .
$$

This is a bounded sequence in $H^{1}\left(\mathbb{R} \times \mathbb{T}^{2}\right)$, thus, up to considering a subsequence, we can assume that it converges weakly to $\varphi \in H^{1}\left(\mathbb{R} \times \mathbb{T}^{2}\right)$. We then define the profile corresponding to $\mathcal{O}$ as $\widetilde{\varphi}_{\mathcal{O}, k}$. By its definition and (6.6), $\varphi$ has norm smaller than $E$. Besides, we also have that

$$
\frac{\nu}{2} \lesssim \lim _{k \rightarrow+\infty}\left\langle f_{k}, \widetilde{\psi}_{\mathcal{O}, k}\right\rangle_{H^{1} \times H^{1}} \lesssim \lim _{k \rightarrow+\infty}\left\langle g_{k}, \psi\right\rangle_{H^{1} \times H^{1}}=\langle\varphi, \psi\rangle_{H^{1} \times H^{1}}
$$

Consequently, we get that

$$
\|\varphi\|_{H^{1}} \gtrsim \nu
$$

We also observe that since $g_{k}-\varphi$ weakly converges to 0 in $H^{1}$, there holds that

$$
\begin{aligned}
\left\|A f_{k}\right\|_{L^{2}}^{2} & =\left\|A g_{k}\right\|_{L^{2}}^{2}=\left\|A\left(g_{k}-\varphi\right)\right\|_{L^{2}}^{2}+\|A \varphi\|_{L^{2}}^{2}+o_{k}(1) \\
& =\left\|A\left(f_{k}-\varphi_{\mathcal{O}, k}\right)\right\|_{L^{2}}^{2}+\|A \varphi\|_{L^{2}}^{2}+o_{k}(1)
\end{aligned}
$$

for $A=1$ or $A=\nabla_{x, y}$.

The situation if $\mathcal{O}$ is a Euclidean frame is similar. In this case, for $k$ large enough, we consider

$$
\varphi_{k}(y)=N_{k}^{-\frac{1}{2}} \eta^{3}\left(y /\left(10 N_{k}^{\frac{1}{2}}\right)\right)\left(\Pi_{\left(-t_{k},-x_{k}\right)} f_{k}\right)\left(\Psi\left(y / N_{k}\right)\right), \quad y \in \mathbb{R}^{3} .
$$

This is a sequence of functions bounded in $\dot{H}^{1}\left(\mathbb{R}^{3}\right)$. We can thus extract a subsequence that converges weakly to a function $\varphi \in \dot{H}^{1}\left(\mathbb{R}^{3}\right)$ satisfying

$$
\|\varphi\|_{\dot{H}^{1}\left(\mathbb{R}^{3}\right)} \lesssim 1
$$

Now, let $\gamma \in C_{0}^{\infty}\left(\mathbb{R}^{3}\right)$; for $k$ large enough,

$$
\begin{aligned}
\left\langle f_{k}, \widetilde{\gamma}_{\mathcal{O}, k}\right\rangle_{H^{1} \times H^{1}\left(\mathbb{R} \times \mathbb{T}^{2}\right)} & =\left\langle\Pi_{-\left(t_{k}, x_{k}\right)} f_{k}, T_{N_{k}}^{e} \gamma\right\rangle_{H^{1} \times H^{1}\left(\mathbb{R} \times \mathbb{T}^{2}\right)} \\
& =\langle\varphi, \gamma\rangle_{\dot{H}^{1} \times \dot{H}^{1}\left(\mathbb{R}^{3}\right)}+o_{k}(1) .
\end{aligned}
$$

Form this and (6.11), (6.12), we conclude that

$$
\|\varphi\|_{\dot{H}^{1}\left(\mathbb{R}^{3}\right)} \gtrsim \nu
$$

and that

$$
h_{k}=f_{k}-\widetilde{\varphi}_{\mathcal{O}, k}
$$

is absent from the frame $\mathcal{O}$. Now, similarly to (6.14) and using Lemma 6.2 we see that

$$
\begin{aligned}
\left\|h_{k}\right\|_{L^{2}}^{2} & =\left\|f_{k}\right\|_{L^{2}}^{2}+o_{k}(1) \\
\left\|\nabla_{x, y} h_{k}\right\|_{L^{2}}^{2} & =\left\|\nabla_{x, y} f_{k}\right\|_{L^{2}}^{2}+\|\nabla \varphi\|_{L^{2}\left(\mathbb{R}^{3}\right)}^{2}-2\left\langle\nabla f_{k}, \nabla \widetilde{\varphi}_{\mathcal{O}, k}\right\rangle_{L^{2} \times L^{2}} \\
& =\left\|\nabla_{x, y} f_{k}\right\|_{L^{2}}^{2}-\|\nabla \varphi\|_{L^{2}\left(\mathbb{R}^{3}\right)}^{2}+o_{k}(1) .
\end{aligned}
$$


Defining $f_{k}^{0}=\phi_{k}$ and for each $\alpha, f_{k}^{\alpha+1}=f_{k}^{\alpha}-\widetilde{\psi}_{\mathcal{O}^{\alpha}, k}$ where $\widetilde{\psi}_{\mathcal{O}^{\alpha}, k}$ is the profile given by the considerations above; iterating this claim at most $O\left(\nu^{-2}\right)$ times and replacing $\phi_{k}$ by

$$
\phi_{k}^{\prime}:=\phi_{k}-\sum_{\alpha} \widetilde{\psi}_{\mathcal{O}^{\alpha}, k}
$$

we obtain that $\left\{\phi_{k}^{\prime}\right\}$ satisfies

$$
\limsup _{k \rightarrow+\infty}\left\|\phi_{k}^{\prime}\right\|_{H^{1}} \leq E<+\infty
$$

and

$$
\limsup _{k \rightarrow+\infty} \sup _{N \geq 1, t, x, y} N^{-\frac{1}{2}}\left|\left(e^{i t \Delta} P_{N} \phi_{k}^{\prime}\right)(x, y)\right|<\nu .
$$

This proves our lemma.

We can now finish the proof of Lemma 6.4

Proof of Lemma 6.4. First, for $\nu=\nu(\delta, E)$ to be decided later, we run Lemma 6.5 and extract some profiles. Then, we replace $\phi_{k}$ by $\phi_{k}^{\prime}$, thus ensuring that (6.10) holds for the sequence $\left\{\phi_{k}^{\prime}\right\}_{k}$. We now consider

$$
\Lambda_{0}\left(\left\{\phi_{k}^{\prime}\right\}\right)=\limsup _{k \rightarrow+\infty}\left\|e^{i t \Delta} \phi_{k}^{\prime}\right\|_{Z(\mathbb{R})} .
$$

If $\Lambda_{0}\left(\left\{\phi_{k}^{\prime}\right\}\right)<\delta$, we may set $R_{k}^{A}=\phi_{k}^{\prime}$ for all $k$ and we get Lemma 6.4 Assume otherwise.

We claim that if $\left\{\phi_{k}^{\prime}\right\}_{k}$ satisfies $\Lambda_{0}\left(\left\{\phi_{k}^{\prime}\right\}\right) \geq \delta$ and $\left\{\phi_{k}^{\prime}\right\}$ is orthogonal to a family of frames $\mathcal{O}^{\alpha}, 1 \leq \alpha \leq A$, then there exists a frame $\mathcal{O}$ orthogonal to $\mathcal{O}^{\alpha}, 1 \leq \alpha \leq A$ and an associated profile $\widetilde{\varphi}_{\mathcal{O}, k}$ such that, after passing to a subsequence, we have that

$$
\limsup _{k \rightarrow+\infty}\left\|\widetilde{\varphi}_{\mathcal{O}, k}\right\|_{H^{1}\left(\mathbb{R} \times \mathbb{T}^{2}\right)} \gtrsim_{\delta} 1, \quad \phi_{k}^{\prime}-\widetilde{\varphi}_{\mathcal{O}, k} \text { is absent from } \mathcal{O} .
$$

Once this claim is established, the end of the proof follows by iterating the extraction process as in Lemma 6.5.

Since $\Lambda_{0}\left(\left\{\phi_{k}^{\prime}\right\}\right) \geq \delta$, by Hölder's inequality and Strichartz estimates (3.2), we have that, for $p_{0} \in\{9 / 2,18\}$ and $q_{0}=\frac{4 p_{0}}{p_{0}-2}$,

$$
\begin{aligned}
c_{N}^{k} & =N^{\frac{5}{p_{0}}-\frac{1}{2}}\left\|e^{i t \Delta} P_{N} \phi_{k}^{\prime}\right\|_{l_{\gamma}^{q_{0}} L_{x, y, t}^{p_{0}}\left(\mathbb{R} \times \mathbb{T}^{2} \times I_{\gamma}\right)} \\
\left\|c_{N}^{k}\right\|_{l_{N}^{p_{0}}} & \leq\left\|c_{N}^{k}\right\|_{l_{N}^{\frac{2}{p_{0}}}}^{p_{N}}\left\|c_{N}^{k}\right\|_{l_{N}^{\frac{p_{0}-2}{p_{0}}}}^{p_{0}} \leq\left(\sum_{N \geq 1} N^{2}\left\|P_{N} \phi_{k}^{\prime}\right\|_{L^{2}}^{2}\right)^{\frac{1}{p_{0}}}\left(\sup _{N} c_{N}^{k}\right)^{\frac{p_{0}-2}{p_{0}}} .
\end{aligned}
$$

Using (6.16), we obtain that there exists a sequence of scales $N_{k} \geq 1$ such that, for some $p_{0}$ in $\{9 / 2,18\}$,

$$
(\delta / 2)^{\frac{p_{0}}{p_{0}-2}}<\Lambda_{0}\left(\left\{\phi_{k}^{\prime}\right\}\right)^{\frac{p_{0}}{p_{0}-2}} \leq E^{\frac{2}{p_{0}-2}} N_{k}^{\frac{5}{p_{0}}-\frac{1}{2}}\left\|e^{i t \Delta} P_{N_{k}} \phi_{k}^{\prime}\right\|_{l_{\gamma}^{q_{0}} L_{x, y, t}^{p_{0}}\left(\mathbb{R} \times \mathbb{T}^{2} \times I_{\gamma}\right)} .
$$

We conclude that there exists a sequence $h_{k} \in C_{c}^{\infty}\left(\mathbb{R}_{x} \times \mathbb{T}_{y}^{2} \times \mathbb{R}_{t}\right)$ such that

$$
\begin{aligned}
& 1 \leq\left\|h_{k}\right\|_{l_{\gamma}^{q_{0}} L_{x, y, t}^{p_{0}^{\prime}}\left(\mathbb{R} \times \mathbb{T}^{2} \times I_{\gamma}\right)} \leq 2 \\
& (\delta / 2)^{\frac{p_{0}}{p_{0}-2}} E^{-\frac{2}{p_{0}-2}} N_{k}^{\frac{1}{2}-\frac{5}{p_{0}}} \leq\left\langle h_{k}, e^{i t \Delta} P_{N_{k}} \phi_{k}^{\prime}\right\rangle_{L^{2} \times L_{x, y, t, \gamma}^{2}} .
\end{aligned}
$$


Now, for a given threshold $B$, we introduce the partition function

$$
\chi_{B}(\gamma)= \begin{cases}1 & \text { if }\left\|h_{k}\right\|_{L_{x, y, t}^{p_{0}^{\prime}}\left(\mathbb{R} \times \mathbb{T}^{2} \times I_{\gamma}\right)} \geq B \\ 0 & \text { otherwise }\end{cases}
$$

and we decompose

$$
h_{k}(x, y, t)=h_{k}^{>B}+h_{k}^{<B}=h_{k}(x, y, t) \chi_{B}\left(\left[\frac{t}{2 \pi}\right]\right)+h_{k}(x, y, t)\left(1-\chi_{B}\left(\left[\frac{t}{2 \pi}\right]\right)\right)
$$

so that

$$
\begin{aligned}
& \left\|h_{k}\right\|_{l_{\gamma}^{q_{0}^{\prime}} L_{x, y, t}^{p_{0}^{\prime}}\left(\mathbb{R} \times \mathbb{T}^{2} \times I_{\gamma}\right)} \leq\left\|h_{k}^{>B}\right\|_{l_{\gamma}^{q_{0}^{\prime}} L_{x, y, t}^{p_{0}^{\prime}}\left(\mathbb{R} \times \mathbb{T}^{2} \times I_{\gamma}\right)}+\left\|h_{k}^{<B}\right\|_{l_{\gamma}^{q_{0}^{\prime}} L_{x, y, t}^{p_{0}^{\prime}}\left(\mathbb{R} \times \mathbb{T}^{2} \times I_{\gamma}\right)} \leq B . \\
& \sup _{\gamma}\left\|h_{k}^{<B}\right\|_{L_{x, y, t}^{p_{0}^{\prime}}\left(\mathbb{R} \times \mathbb{T}^{2} \times I_{\gamma}\right)} \leq B .
\end{aligned}
$$

Using Strichartz estimates, we have that, for $p_{1}=17 / 4<p_{0}$,

$$
\limsup _{k \rightarrow+\infty} N_{k}^{\frac{5}{p_{1}}-\frac{1}{2}}\left\|e^{i t \Delta} P_{N_{k}} \phi_{k}^{\prime}\right\|_{l_{\gamma}^{\frac{p_{1}}{p_{1}-2}} L_{x, y, t}^{p_{1}}\left(\mathbb{R} \times \mathbb{T}^{2} \times I_{\gamma}\right)} \lesssim \limsup _{k \rightarrow+\infty}\left\|\phi_{k}^{\prime}\right\|_{H^{1}} \lesssim E .
$$

Interpolating with (6.17), we obtain that

$$
\limsup _{k \rightarrow+\infty} N_{k}^{\frac{5}{p_{0}}-\frac{1}{2}}\left\|e^{i t \Delta} P_{N_{k}} \phi_{k}^{\prime}\right\|_{l_{\gamma}^{\frac{4 p_{0}}{p_{1}-2}} L_{x, y, t}^{p_{0}}\left(\mathbb{R} \times \mathbb{T}^{2} \times I_{\gamma}\right)} \lesssim E^{\frac{p_{1}}{p_{0}}} \nu^{\frac{p_{0}-p_{1}}{p_{0}}} .
$$

We first observe that

$$
\left|\operatorname{supp}_{\gamma}\left(h_{k}^{>B}\right)\right| \leq(2 / B)^{q_{0}^{\prime}}
$$

and consequently, by Hölder's inequality in $\gamma$,

$$
\begin{aligned}
\left\langle e^{i t \Delta} \phi_{k}^{\prime}, h_{k}^{>B}\right\rangle & \leq\left\|e^{i t \Delta} \phi_{k}^{\prime}\right\|_{l_{\gamma}^{\frac{4 p_{0}}{p^{2}}}} L_{x, y, t}^{p_{0}\left(I_{\gamma}\right)}\left\|h_{k}^{>B}\right\|_{l_{\gamma}^{q_{0}^{\prime}} L_{x, y, t}^{p_{0}^{\prime}\left(I_{\gamma}\right)}}\left[(2 / B)^{q_{0}^{\prime}}\right]^{\frac{p_{0}-p_{1}}{4 p_{0}}} \\
& \lesssim 2[2 / B]^{\frac{p_{0}-p_{1}}{3 p_{0}+2}} E^{\frac{p_{1}}{p_{0}}} \nu^{\frac{p_{0}-p_{1}}{p_{0}}} N_{k}^{\frac{1}{2}-\frac{5}{p_{0}}} .
\end{aligned}
$$

Consequently, for any fixed $B>0$, we can choose $\nu=\nu(B, \delta)$ with

$$
\nu^{\frac{1}{18}}=c E^{-\frac{2}{p_{0}-2}-\frac{p_{1}}{p_{0}}} \delta^{\frac{p_{0}}{p_{0}-2}} B^{\frac{p_{0}-p_{1}}{3 p_{0}+2}},
$$

for $c>0$ a universal constant sufficiently small so that,

$$
\begin{aligned}
(\delta / 2)^{\frac{p_{0}}{p_{0}-2}} E^{-\frac{2}{p_{0}-2}} N_{k}^{\frac{1}{2}-\frac{5}{p_{0}}} & \leq 2\left\langle h_{k}^{<B}, e^{i t \Delta} P_{N_{k}} \phi_{k}^{\prime}\right\rangle_{L^{2} \times L_{x, y, t, \gamma}^{2}} \\
& \leq 2 E N_{k}^{-1}\left\|\int_{\mathbb{R}} e^{-i s \Delta} P_{N_{k}} h_{k}^{<B}(x, y, s) d s\right\|_{L_{x, y}^{2}\left(\mathbb{R} \times \mathbb{T}^{2}\right)} .
\end{aligned}
$$

Using Strichartz estimates (3.4), we obtain that

$$
\begin{aligned}
\left\|\int_{\mathbb{R}} e^{-i s \Delta} P_{N_{k}} h_{k}^{<B}(x, y, s) d s\right\|_{L_{x, y}^{2}} & \lesssim N_{k}^{\frac{3}{2}-\frac{5}{p_{0}}}\left\|h_{k}^{<B}\right\|_{l_{\gamma}^{2} L_{x, y, t}^{p_{0}^{\prime}}\left(I_{\gamma}\right)}+N_{k}^{1-\frac{3}{p_{0}}}\left\|h_{k}^{<B}\right\|_{l_{\gamma}^{q_{0}^{\prime}} L_{x, y, t}^{p_{0}^{\prime}}} \\
& \lesssim N_{k}^{\frac{3}{2}-\frac{5}{p_{0}}}\left\|h_{k}^{<B}\right\|_{l_{\gamma} \frac{2 p_{0}}{3 p_{0}+2}}^{l_{q_{0}^{\prime}}^{p_{0}^{\prime}} L_{x, y, t}^{p_{0}^{\prime}}\left(I_{\gamma}\right)} \\
& B^{\frac{p_{0}+2}{3 p_{0}+2}}+N_{k}^{\frac{p_{0}-3}{p_{0}}}\left\|h_{k}^{<B}\right\|_{l_{\gamma}^{q_{0}^{\prime}} L_{x, y, t}^{p_{0}^{\prime}}\left(I_{\gamma}\right)} \\
& \lesssim N_{k}^{\frac{3}{2}-\frac{5}{p_{0}}} B^{\frac{p_{0}+2}{3 p_{0}+2}}+N_{k}^{\frac{p_{0}-3}{p_{0}}} .
\end{aligned}
$$


Choosing $B$ such that

$$
B^{\frac{p_{0}+2}{3 p_{0}+2}}=\varepsilon E^{-\frac{2}{p_{0}-2}} \delta^{\frac{p_{0}}{p_{0}-2}}
$$

for some absolute constant $\varepsilon>0$ small enough and plugging into (6.19), we obtain that

$$
\delta^{\frac{p_{0}}{p_{0}-2}} \lesssim \varepsilon \delta^{\frac{p_{0}}{p_{0}-2}}+E^{\frac{2}{p_{0}-2}} N_{k}^{\frac{2}{p_{0}}-\frac{1}{2}} .
$$

If $\varepsilon>0$ is small enough, we obtain a uniform bound

$$
N_{k} \lesssim\left[E^{\frac{2}{p_{0}-2}} \delta^{-\frac{p_{0}}{p_{0}-2}}\right]^{\frac{2 p_{0}}{p_{0}-4}}
$$

To sum up, we have proved that if $\Lambda_{0}\left(\left\{\phi_{k}^{\prime}\right\}\right)>\delta$, then there exists a sequence of scales $\left\{N_{k}\right\}$ satisfying (6.20) and such that

$$
N_{k}^{\frac{5}{p_{0}}-\frac{1}{2}}\left\|P_{N_{k}} e^{i t \Delta} \phi_{k}^{\prime}\right\|_{l_{\gamma}^{q_{0}} L_{x, y, t}^{p_{0}}\left(I_{\gamma}\right)}>\delta^{\frac{p_{0}}{p_{0}-2}} E^{-\frac{2}{p_{0}-2}} .
$$

Using again Strichartz estimates, we see that

$$
N_{k}^{\frac{5}{p_{2}}-\frac{1}{2}}\left\|P_{N_{k}} e^{i t \Delta} \phi_{k}^{\prime}\right\|_{l_{\gamma}^{\frac{4 p_{2}}{p_{2}-2}} L_{x, y, t}^{p_{2}}\left(I_{\gamma}\right)} \lesssim E
$$

for $p_{2} \in\{4.001,1000\}$ and using Hölder's estimate, we deduce that

$$
\left\|P_{N_{k}} e^{i t \Delta} \phi_{k}^{\prime}\right\|_{L_{x, y, t}^{6}\left(\mathbb{R} \times \mathbb{T}^{2} \times \mathbb{R}\right)}>c(\delta, E)
$$

for some $c(\delta, E)>0$. Here and below, $c(\delta, E)$ denotes a small positive constant depending only on $\delta$ and $E$ which is allowed to change from line to line.

At this point, we write

$$
P_{N_{k}} e^{i t \Delta_{\mathbb{R} \times \mathbb{T}^{2}}} \phi_{k}^{\prime}(x, y)=\sum_{z=\left(z_{1}, z_{2}\right) \in \mathbb{Z}^{2} ;\left|z_{i}\right| \leq N_{k}} e^{-i t|z|^{2}} e^{i\langle z, y\rangle} \eta_{N_{k}}^{2}(z)\left[e^{i t \Delta_{\mathbb{R}}} \phi_{z, k}^{\prime \prime}(x)\right]
$$

where

$$
\phi_{z, k}^{\prime \prime}(x)=\frac{1}{(2 \pi)^{2}} \int_{\mathbb{T}^{2}} P_{N_{k}} \phi_{k}^{\prime}(x, y) e^{-i\langle z, y\rangle} d y
$$

and, extracting a subsequence, we conclude that there exists $z$ such that, for all $k$,

$$
\begin{aligned}
& \operatorname{supp}\left(\mathcal{F}_{x} \phi_{z, k}^{\prime \prime}\right) \subset\left[-3 N_{k}, 3 N_{k}\right] \\
&\left\|e^{i t \Delta_{\mathbb{R}}} \phi_{z, k}^{\prime \prime}\right\|_{L_{x, t}^{6}(\mathbb{R} \times \mathbb{R})}>c(\delta, E) \\
&\left\|\phi_{z, k}^{\prime \prime}\right\|_{H^{1}(\mathbb{R})} \leq M .
\end{aligned}
$$

To proceed, we borrow the following proposition from [5, 15, 51] (we use the version in [51]):

Proposition 6.6 ([5, 15, 51]). For any $M, c(\delta, E)>0$, there exists a finite set $\mathcal{C} \subset L^{2}(\mathbb{R})$ of functions satisfying,

$$
\|v\|_{L^{2}(\mathbb{R})}=1, \quad\|v\|_{L^{1}(\mathbb{R})} \leq S(E, M, \delta), \forall v \in \mathcal{C}
$$

and $\kappa(M, c(\delta, E))>0$ such that whenever $u \in L^{2}(\mathbb{R})$ obeys the bounds

$$
\|u\|_{L^{2}} \leq M, \quad\left\|e^{i t \Delta_{\mathbb{R}}} u\right\|_{L_{x, t}^{6}(\mathbb{R} \times \mathbb{R})} \geq c(\delta, E),
$$

then there exists $v \in \mathcal{C}$ and $\left(\lambda, \xi_{0}, t_{0}, x_{0}\right) \in \mathbb{R}_{+} \times \mathbb{R} \times \mathbb{R} \times \mathbb{R}$ such that

$$
\left\langle u, v^{\prime}\right\rangle_{L^{2} \times L^{2}(\mathbb{R})} \geq \kappa, \quad v^{\prime}(x)=\lambda^{\frac{1}{2}} e^{i x \xi_{0}}\left[e^{i t_{0} \Delta_{\mathbb{R}}} v\right]\left(\lambda\left(x-x_{0}\right)\right) .
$$


This proposition gives, after extraction, a function $v \in L^{2}(\mathbb{R})$ satisfying (6.22) and a sequence $\left(\lambda_{k}, \xi_{k}, t_{k}, x_{k}\right) \in$ $\mathbb{R}_{+} \times \mathbb{R} \times \mathbb{R} \times \mathbb{R}$ such that

$$
\left\langle\phi_{z, k}^{\prime \prime}, v_{k}\right\rangle \geq \kappa, \quad v_{k}(x)=\lambda_{k}^{\frac{1}{2}} e^{i x \xi_{k}}\left[e^{i t_{k} \Delta_{\mathbb{R}}} v\right]\left(\lambda_{k}\left(x-x_{k}\right)\right) .
$$

We may assume that $v$ has a compactly supported Fourier transform.

We now claim that $\lambda_{k}$ and $\left|\xi_{k}\right|$ remain bounded. Indeed, we compute that

$$
\begin{aligned}
\mathcal{F}_{\mathbb{R}} v_{k}(\xi) & =e^{i \xi_{k} x_{k}} e^{-i x_{k} \xi} \lambda_{k}^{-\frac{1}{2}}\left[\mathcal{F}_{\mathbb{R}} e^{i t_{k} \Delta_{\mathbb{R}}} v\right]\left(\frac{\xi-\xi_{k}}{\lambda_{k}}\right) \\
\left\|\langle\xi\rangle^{-1} \mathcal{F}_{\mathbb{R}} v_{k}(\xi)\right\|_{L^{2}\left(\left[-4 N_{k}, 4 N_{k}\right]\right)} & \leq \sqrt{\pi} \lambda_{k}^{-\frac{1}{2}} S(M, E, \delta) \\
\left\|v_{k}\right\|_{H^{-1}(\mathbb{R})}^{2} & \sim \int_{\mathbb{R}}\left[\frac{1}{1+\left|\xi_{k}+\lambda_{k} \eta\right|}\right]^{2}\left|\mathcal{F}_{\mathbb{R}} v\right|^{2}(\eta) d \eta .
\end{aligned}
$$

The second line, together with (6.21) and (6.23) implies that

$$
\kappa \leq \sqrt{\pi} M S(M, E, \delta) \lambda_{k}^{-\frac{1}{2}}
$$

which forces $\lambda_{k} \leq C_{\kappa}(E, M, \delta)=\pi M^{2} \kappa^{-2} S(M, E, \delta)$. Similarly, the third line, together with (6.21) and (6.23), along with the uniform bound on $\lambda_{k}$ and the compactness of the support of $\mathcal{F}_{\mathbb{R}} v$, forces $\left|\xi_{k}\right| \leq C_{\xi}(E, M, \delta)$.

Assume first that $\lambda_{k}$ remains bounded from below. Then, up to extracting a subsequence, we may assume that $\lambda_{k} \rightarrow \lambda_{\infty} \in(0, \infty)$. Similarly, we may assume that $\xi_{k} \rightarrow \xi_{\infty}$. Then, setting

$$
\tilde{\psi}(x, y)=e^{i\langle z, y\rangle} e^{i x \xi_{\infty}} \lambda_{\infty}^{\frac{1}{2}} v\left(\lambda_{\infty} x\right), \quad t_{k}^{\prime}=-\lambda_{k}^{-2} t_{k}, \quad x_{k}^{\prime}=x_{k}+2 t_{k}^{\prime} \xi_{k}
$$

and defining the frame $\mathcal{O}=\left\{\left(1, t_{k}^{\prime},\left(x_{k}^{\prime}, 0\right), 0\right)_{k}\right\}$, we see from (6.23) that

$$
\begin{aligned}
\kappa & \leq\left\langle P_{N_{k}} \phi_{k}^{\prime}, e^{i\langle z, y\rangle} e^{i x \xi_{k}} \lambda_{k}^{\frac{1}{2}}\left[e^{-i t_{k} \Delta_{\mathbb{R}}} v\right]\left(\lambda_{k}\left(x-x_{k}\right)\right)\right\rangle \\
& \leq\left\langle\phi_{k}^{\prime}, e^{i\left(x_{k} \xi_{\infty}-t_{k}^{\prime}\left(|z|^{2}-\left|\xi_{\infty}\right|^{2}\right)\right)} P_{N_{k}} e^{-i t_{k}^{\prime} \Delta_{\mathbb{R} \times \mathbb{T}^{2}}} \tilde{\psi}\left(x-x_{k}^{\prime}\right)\right\rangle+o_{k}(1)
\end{aligned}
$$

Since $1 \leq N_{k} \lesssim 1$ is dyadic, we may assume (up to a subsequence) that $N_{k}=N_{\infty}$. As a result, setting $\psi=P_{N_{\infty}} \tilde{\psi}$, we see that the Scale-1 profile $\widetilde{\psi}_{\mathcal{O}, k}$ satisfies both (6.11) and (6.12). We also conclude that $\mathcal{O}$ is orthogonal to $\mathcal{O}^{\alpha}, 1 \leq \alpha \leq A$. In addition, proceeding as in the proof of Lemma 6.4 we find $\varphi$ satisfying (6.18).

Assume now that $\lambda_{k} \rightarrow 0$. Let $M_{k}$ be a dyadic number such that $1 \leq \lambda_{k}^{-1} M_{k} \leq 2$ and consider the sequence

We have that

$$
\Phi_{k}(x, y)=M_{k}^{-\frac{1}{2}} e^{-i t_{k} \partial_{x x}}\left[e^{i \xi_{k} x / M_{k}} \phi_{k}^{\prime}\left(x_{k}+\frac{x}{M_{k}}, y\right)\right]
$$

$$
\left\|\Phi_{k}\right\|_{L^{2}\left(\mathbb{R} \times \mathbb{T}^{2}\right)}^{2}+\left\|\nabla_{y} \Phi_{k}\right\|_{L^{2}\left(\mathbb{R} \times \mathbb{T}^{2}\right)}^{2} \leq M^{2}+E^{2}
$$

hence, up to extracting a subsequence, we may assume that $\Phi_{k} \rightarrow \Phi$ in $H^{0,1}\left(\mathbb{R} \times \mathbb{T}^{2}\right)$. We define

$$
t_{k}^{\prime}=-M_{k}^{-2} t_{k}, \quad \xi_{k}^{\prime}=-\xi_{k}, \quad x_{k}^{\prime}=x_{k}+2 t_{k}^{\prime} \xi_{k}^{\prime},
$$

and $\mathcal{O}=\left(M_{k}, t_{k}^{\prime},\left(x_{k}^{\prime}, 0\right), \xi_{k}^{\prime}\right)$. Then we obtain from the existence of a nonzero inner product (6.23) that $\mathcal{O}$ is orthogonal to $\mathcal{O}^{\alpha}, 1 \leq \alpha \leq A$ and we see from the definition of $\Phi_{k}$ that (6.18) holds with $\varphi=e^{i \theta_{\infty} \Phi}$ for some $\theta_{\infty} \in \mathbb{R} / \mathbb{Z}$. This finishes the proof. 


\section{INDUCTION ON ENERGY}

7.1. Proof of Theorem 1.3, We are now ready to prove Theorem 1.3. We follow an induction on energy method formalized in [36, 37. Define

$$
\Lambda(L)=\sup \left\{\|u\|_{Z(I)}^{2}: u \in X_{l o c}^{1}(I), E(u)+\frac{1}{2} M(u) \leq L\right\}
$$

where the supremum is taken over all strong solutions of full energy less than $L$. By the local wellposedness theory in Theorem 1.1, this is sublinear in $L$ and finite for $L$ sufficiently small. In addition, define

$$
L_{\max }=\sup \{L: \Lambda(L)<+\infty\} .
$$

Our goal is to prove that $L_{\max }=+\infty$. As in [53], the key proposition is:

Proposition 7.1. Assume that $L_{\max }<+\infty$ and that Conjecture 1.2 holds for $L_{\max }$. Let $\left\{t_{k}\right\}_{k},\left\{a_{k}\right\}_{k}$, $\left\{b_{k}\right\}_{k}$ be arbitrary sequences of real numbers and $\left\{u_{k}\right\}$ be a sequence of solutions to (1.1) such that $u_{k} \in X_{c, l o c}^{1}\left(t_{k}-a_{k}, t_{k}+b_{k}\right)$ and satisfying

$$
L\left(u_{k}\right) \rightarrow L_{\max }, \quad\left\|u_{k}\right\|_{Z\left(t_{k}-a_{k}, t_{k}\right)} \rightarrow+\infty, \quad\left\|u_{k}\right\|_{Z\left(t_{k}, t_{k}+b_{k}\right)} \rightarrow+\infty .
$$

Then, passing to a subsequence, there exists a sequence $x_{k} \in \mathbb{R}$ and $w \in H^{1}\left(\mathbb{R} \times \mathbb{T}^{2}\right)$ such that

$$
w_{k}(x, y)=u_{k}\left(x-x_{k}, y, t_{k}\right) \rightarrow w
$$

strongly in $H^{1}\left(\mathbb{R} \times \mathbb{T}^{2}\right)$.

The proof of this key proposition is postponed to the end of this section.

Corollary 7.2. Assume that $L_{\max }<+\infty$ and that Conjecture 1.2 holds for some $E_{\max }^{l s} \geq L_{\max }$. Then, there exists $u \in X_{\text {loc }}^{1}(\mathbb{R})$ solving (1.1) and a Lipschitz function $\underline{x}: \mathbb{R} \rightarrow \mathbb{R}$ such that $L(u)=L_{\text {max }}$ and

$$
\begin{gathered}
\sup _{t \in \mathbb{R}}\left|\underline{x}^{\prime}(t)\right| \lesssim 1 \\
\{u(x-\underline{x}(t), y, t): t \in \mathbb{R}\} \text { is precompact in } H^{1}\left(\mathbb{R} \times \mathbb{T}^{2}\right) .
\end{gathered}
$$

Proof of Corollary 7.2. Assuming that $L_{\max }<+\infty$, we can find a sequence of solutions of (1.1) $u_{k}$ satisfying (7.1). Applying Proposition [7.1] we can extract a subsequence and obtain a sequence $x_{k}$ such that (7.2) holds for some $w \in H^{1}\left(\mathbb{R} \times \mathbb{T}^{2}\right)$. Clearly $L(w)=L_{\max }$. Let $U \in C\left(I: H^{1}\right)$ be the (maximal) strong solution of (1.1) with initial data $w$, defined on $I=\left(-a_{\infty}, b_{\infty}\right)$. A consequence of the local wellposedness theory and (7.1) is that

$$
\|W\|_{Z\left(-a_{\infty}, 0\right)}=\|W\|_{Z\left(0, b_{\infty}\right)}=+\infty .
$$

We first claim that there exists $\kappa>0$ such that for all $t$ in $I$,

$$
\|U\|_{Z(t-2 \kappa, t+2 \kappa)} \leq 2 .
$$

In particular, $U$ is global, $a_{\infty}=b_{\infty}=\infty$.

Assume (7.5) is not true. Then there exists a sequence $t_{k} \in I$ such that

$$
\|U\|_{Z\left(t_{k}-\frac{1}{k}, t_{k}+\frac{1}{k}\right)} \geq 2 .
$$

We can apply Proposition 7.1 to the sequence $U\left(t_{k}\right)$ and obtain that, up to a subsequence, there exists $x_{k}$ such that $U_{k}(x, y):=U\left(t_{k}, x-x_{k}, y\right) \rightarrow \omega$ in $H^{1}$. Let $W$ be the nonlinear solution of (1.1) with initial data $\omega$. By the local wellposedness theory in Proposition 4.4, there exists $\kappa^{*}>0$ such that

$$
\|W\|_{Z\left(-\kappa^{*}, \kappa^{*}\right)} \leq 1
$$


and still by the stability theory Proposition 4.6, we obtain that, for $k$ large enough,

$$
\|U\|_{Z\left(t_{k}-\kappa^{*}, t_{k}+\kappa^{*}\right)} \leq 2
$$

which gives a contradiction for $k$ large enough.

We now prove (7.3). We define the sequence of times $\left\{t_{k}\right\}=\{k \kappa\}$ and for each $t_{k}$, we define $\underline{x}_{k}$ and $R_{k}$ such that

$$
\frac{1}{2} \int_{\left\{\left|x-\underline{x}_{k}\right| \leq R_{k}\right\}} \int_{\mathbb{T}^{2}}\left[\left|u\left(t_{k}, x, y\right)\right|^{2}+\left|\nabla u\left(t_{k}, x, y\right)\right|^{2}+\frac{1}{3}\left|u\left(t_{k}, x, y\right)\right|^{6}\right] d x d y=99 / 100 L_{\max }
$$

and $R_{k}$ is minimal with this property. While $\underline{x}_{k}$ is not necessarily unique, we claim that there exists $D$ such that for all $k$,

and that

$$
R_{k} \leq D, \quad\left|\underline{x}_{k}-\underline{x}_{k+1}\right| \leq D
$$

$$
\left\{u\left(t_{k}+s, x-\underline{x}_{k}\right), k \in \mathbb{Z}, s \in(-\kappa, \kappa)\right\} \quad \text { is precompact in } H^{1}\left(\mathbb{R} \times \mathbb{T}^{2}\right) .
$$

The fact that the $R_{k}$ are uniformly bounded is a direct consequence of compactness up to translation of $\left\{u\left(t_{k}\right)\right\}_{k}$. Assume now that $\left\{v_{k}(x, y)=u\left(x-\underline{x}_{k}, y, t_{k}\right)\right\}_{k}$ was not precompact in $H^{1}\left(\mathbb{R} \times \mathbb{T}^{2}\right)$. Then, we could find $\varepsilon>0$ and a subsequence $k^{\prime}$ such that for all $k_{1}^{\prime}, k_{2}^{\prime}$,

$$
\left\|v_{k_{1}}-v_{k_{2}{ }^{\prime}}\right\|_{H^{1}\left(\mathbb{R} \times \mathbb{T}^{2}\right)}>\varepsilon \text {. }
$$

On the other hand, applying once again Proposition 7.1. we see that there exists a sequence $\bar{x}^{k}$ and a subsequence of $k^{\prime}$ such that

$$
v_{k^{\prime \prime}}\left(x-\bar{x}^{k^{\prime \prime}}, y\right) \rightarrow w(x, y) \quad \text { strongly in } H^{1}\left(\mathbb{R} \times \mathbb{T}^{2}\right) .
$$

Necessarily, from (7.6),$\left\{\bar{x}^{k}\right\}_{k}$ remains bounded, so that the convergence of $\left\{v_{k^{\prime \prime}}\right\}_{k}$ contradicts (7.9). Using (7.5) and the precompactness of $\left\{v_{k}\right\}_{k}$, we obtain (7.8). For the same reason as above, this implies the second statement in (7.7). Choosing $\underline{x}(t)$ to be a Lipschitz function satisfying $\underline{x}\left(t_{k}\right)=\underline{x}_{k}$, we obtain (7.3). This ends the proof.

Now, we can finish the proof of our main theorem.

Proposition 7.3. Assume that $u$ satisfies the conclusions of Corollary 7.2, then $u=0$. In particular, $L_{\max } \geq E_{\max }^{l s}$.

Proof. Assume that $u \neq 0$. Then, from the compactness property (7.3), we see that there exists $\rho>0$ such that

$$
\inf _{t \in \mathbb{R}} \min \left(\|u(t)\|_{L_{x, y}^{6}\left(\mathbb{R} \times \mathbb{T}^{2}\right)},\|u(t)\|_{L_{x, y}^{2}\left(\mathbb{R} \times \mathbb{T}^{2}\right)}\right) \geq \rho .
$$

The proof we use is adapted from an argument of Duyckaerts-Holmer-Roudenko [23] (see also [45]). The fundamental tool is a monotonicity formula derived from the conserved momentum

$$
\operatorname{Mom}(u)=\operatorname{Im} \int_{\mathbb{R} \times \mathbb{T}^{2}} \bar{u}(x, y, t) \nabla u(x, y, t) d x d y
$$

First, replacing $u$ by 12

$$
\begin{aligned}
& v(z, t)=e^{-i\left|\xi_{0}\right|^{2} t+i\left\langle z, \xi_{0}\right\rangle} u\left(z-2 \xi_{0} t, t\right), \quad M(u) \xi_{0}=-\operatorname{Mom}(u) \\
& M(v)=M(u), \quad E(u)=E(v)+\left|\xi_{0}\right|^{2} M(u)
\end{aligned}
$$

\footnotetext{
${ }^{12} \xi_{0}$ is well defined since $M(u) \neq 0$ by (7.10).
} 
we may assume that $u$ satisfies

$$
\operatorname{Mom}(u)=0
$$

in addition to (7.3).

Define the Virial action by

$$
A_{R}(t)=\int_{\mathbb{R} \times \mathbb{T}^{2}} \chi_{R}(x-\underline{x}(t)) \cdot(x-\underline{x}(t)) \cdot \operatorname{Im}\left[\bar{u}(x, y, t) \partial_{x} u(x, y, t)\right] d x d y
$$

for $\chi_{R}(x)=\chi(x / R)$ and $\chi$ satisfies $\chi(x)=1$ when $|x| \leq 1$ and $\chi(x)=0$ when $|x| \geq 2$. Clearly

$$
\sup _{t}\left|A_{R}(t)\right| \lesssim R
$$

Independently, we compute that

$$
\begin{aligned}
\frac{d}{d t} A_{R}= & -\underline{x}^{\prime}(t) \operatorname{Im} \int_{\mathbb{R} \times \mathbb{T}^{2}} \bar{u}(x, y, t) \partial_{x} u(x, y, t) d x d y \\
& -\underline{x}^{\prime}(t) \int_{\mathbb{R} \times \mathbb{T}^{2}}\left\{\left(\chi^{\prime}\right)_{R}(x-\underline{x}(t)) \frac{x-\underline{x}(t)}{R}-\left(1-\chi_{R}(x-\underline{x})\right)\right\} \operatorname{Im}\left[\bar{u}(x, y, t) \partial_{x} u(x, y, t)\right] d x d y \\
& +\int_{\mathbb{R} \times \mathbb{T}^{2}} \chi_{R}(x-\underline{x}(t))(x-\underline{x}(t)) \cdot \partial_{t} \operatorname{Im}\left[\bar{u}(x, y, t) \partial_{x} u(x, y, t)\right] d x d y .
\end{aligned}
$$

The first line vanishes thanks to assumption (7.11). The second line can be bounded by

$$
\int_{\{|x-\underline{x}(t)| \geq R\}} \int_{\mathbb{T}^{2}}\left[|u(x, y, t)|^{2}+|\nabla u(x, y, t)|^{2}\right] d x d y=O_{R}(t)
$$

where, thanks to (7.3),

$$
\sup _{t} O_{R}(t) \rightarrow 0 \text { as } R \rightarrow+\infty
$$

Since

we get that

$$
\partial_{t} \operatorname{Im}\left[\bar{u}(x, y, t) \partial_{x} u(x, y, t)\right]=\partial_{x} \Delta \frac{|u|^{2}}{2}-2 \operatorname{div}\left\{\operatorname{Re}\left\{\partial_{x} \bar{u} \nabla u\right\}\right\}-\frac{4}{6} \partial_{x}|u|^{6},
$$

$$
\begin{aligned}
\frac{d}{d t} A_{R} & =4 \int_{\mathbb{R} \times \mathbb{T}^{2}} \chi_{R}(x-\underline{x}(t))\left\{\frac{1}{2}\left|\partial_{x} u(x, y, t)\right|^{2}+\frac{1}{6}|u(x, y, t)|^{6}\right\} d x d y \\
& +4 \int_{\mathbb{R} \times \mathbb{T}^{2}}\left(\chi^{\prime}\right)_{R}(x-\underline{x}(t)) \frac{x-\underline{x}(t)}{R}\left\{\frac{1}{2}\left|\partial_{x} u(x, y, t)\right|^{2}+\frac{1}{6}|u(x, y, t)|^{6}\right\} d x d y \\
& -\int_{\mathbb{R} \times \mathbb{T}^{2}} \frac{|u(x, y, t)|^{2}}{2} \partial_{x}^{3}\left[(x-\underline{x}(t)) \chi_{R}(x-\underline{x}(t))\right] d x d y+O_{R}(t) \\
& =4 \int_{{\mathbb{R} \times \mathbb{T}^{2}}^{2}}\left\{\frac{1}{2}\left|\partial_{x} u(x, y, t)\right|^{2}+\frac{1}{6}|u(x, y, t)|^{6}\right\} d x d y+\tilde{O}_{R}(t)
\end{aligned}
$$

where $\tilde{O}_{R}(t)$ satisfies the same bounds as $O_{R}(t)$ in (7.13). Integrating this equality, we obtain

$$
\left|A_{R}(t)-A_{R}(0)\right| \geq 4 t \rho-t \sup _{t} \tilde{O}_{R}(t) .
$$

Taking $R$ sufficiently large and using (7.13), we obtain, when $t$ is sufficiently large a contradiction with (17.12). This finishes the proof. 


\subsection{Proof of Proposition 7.1.}

Proof. Without loss of generality, we may assume that $t_{k}=0$. We apply Proposition 6.3 to the sequence $\left\{u_{k}(0)\right\}_{k}$ which is indeed bounded in $H^{1}\left(\mathbb{R} \times \mathbb{T}^{2}\right)$. This way we obtain, for all $J$,

$$
u_{k}(0)=\sum_{1 \leq \alpha \leq J} \widetilde{\varphi}_{\mathcal{O}^{\alpha}, k}^{\alpha}+\sum_{1 \leq \beta \leq J} \widetilde{\psi}_{\mathcal{S}^{\beta}, k}^{\beta}+\sum_{1 \leq \gamma \leq J} \widetilde{\omega}_{\mathcal{O}^{\gamma}, k}^{\gamma}+R_{k}^{J} .
$$

Case I: There are no profiles. Then, taking $J$ sufficiently large, we see from (6.4) that

$$
\left\|e^{i t \Delta} u_{k}(0)\right\|_{Z(\mathbb{R})}=\left\|e^{i t \Delta} R_{k}^{J}\right\|_{Z(\mathbb{R})} \leq \delta_{0} / 2
$$

for $k$ sufficiently large, where $\delta_{0}=\delta_{0}\left(L_{\max }\right)$ is given in Proposition 4.4 Then we see from Proposition 4.4 that $u_{k}$ can be extended on $\mathbb{R}$ and that

$$
\lim _{k \rightarrow+\infty}\left\|u_{k}\right\|_{Z(\mathbb{R})} \leq \delta_{0}
$$

which contradicts (7.1).

Hence, we see that there exists at least one profile. Using Lemma 6.2 and passing to a subsequence, we may renormalize every Euclidean profile, that is, up to passing to an equivalent profile, we may assume that every Euclidean frame $\mathcal{E}^{\alpha}$ belongs to $\widetilde{\mathcal{F}}_{e}$, every large-scale frame $\mathcal{S}^{\beta}$ belongs to $\widetilde{\mathcal{F}}_{l s}$, and that every Scale-1 frame $\mathcal{O}^{\gamma}$ belong to $\widetilde{\mathcal{F}}_{1}$, where $\widetilde{\mathcal{F}}_{e}$ was defined in (5.10), $\widetilde{\mathcal{F}}_{l s}$ was defined in (5.31), and

$$
\widetilde{\mathcal{F}}_{1}:=\left\{\left(1, t_{k}, p_{k}, 0\right)_{k}: \text { either }\left|t_{k}\right| \rightarrow+\infty \text { or } t_{k} \equiv 0\right\} .
$$

Besides, passing to a subsequence once again and applying Lemma 6.2, we may assume that for every $\alpha \neq \beta$, either $N_{k}^{\alpha} / N_{k}^{\beta}+N_{k}^{\beta} / N_{k}^{\alpha} \rightarrow+\infty$ as $k \rightarrow+\infty$ or $N_{k}^{\alpha}=N_{k}^{\beta}$ for all $k$ and in this case, either $t_{k}^{\alpha}=t_{k}^{\beta}$ as $k \rightarrow+\infty$ or $\left(N_{k}^{\alpha}\right)^{2}\left|t_{k}^{\alpha}-t_{k}^{\beta}\right| \rightarrow+\infty$ as $k \rightarrow+\infty$.

Now for every linear profile, we define the associated nonlinear profile as the maximal solution of (1.1) with the same initial data. More precisely,

(1) If $\mathcal{E}^{\alpha}$ is a Euclidean frame, this is given by Lemma 5.4. This nonlinear profile is noted $U_{k}^{E, \alpha}$.

(2) If $\mathcal{S}^{\beta}$ is a large-scale profile, this is given by Lemma 5.9. This nonlinear profile is noted $U_{k}^{l s, \beta}$.

(3) If $\mathcal{O}^{\gamma}=\left(1, t^{\gamma}, x_{k}^{\gamma}\right)_{k}$ is a Scale- 1 frame, then letting $W^{\gamma}$ be the maximal strong solution with initial data $\omega^{\gamma} \in H^{1}\left(\mathbb{R} \times \mathbb{T}^{2}\right)$ if $t_{k}^{\gamma} \equiv 0$, and $W^{\gamma}$ be the unique nonlinear solution which scatters to $e^{i t \Delta_{\mathbb{R} \times \mathbb{T}^{2}} \omega^{\gamma}}$ as $t \rightarrow \pm \infty$ if $t_{k} \rightarrow \mp \infty$, we have that

$$
U_{k}^{1, \gamma}(x, t)=W^{\gamma}\left(x-x_{k}^{\gamma}, t-t_{k}^{\gamma}\right)
$$

From (6.14) and defining

$$
\begin{aligned}
& L_{E}(\alpha):=\lim _{k \rightarrow+\infty}\left(E\left(\widetilde{\varphi}_{\mathcal{E}^{\alpha}, k}^{\alpha}\right)+\frac{1}{2} M\left(\widetilde{\varphi}_{\mathcal{E}^{\alpha}, k}^{\alpha}\right)\right)=E_{\mathbb{R}^{3}}\left(\varphi^{\alpha}\right) \in\left(0, L_{\text {max }}\right], \\
& L_{l s}(\beta):=\lim _{k \rightarrow+\infty}\left(E\left(\widetilde{\psi}_{\mathcal{S}^{\beta}, k}^{\beta}\right)+\frac{1}{2} M\left(\widetilde{\psi}_{\mathcal{S}^{\beta}, k}^{\beta}\right)\right)=\left\|\psi^{\beta}\right\|_{H^{0,1}\left(\mathbb{R} \times \mathbb{T}^{2}\right)}^{2} \in\left(0, L_{\text {max }}\right], \\
& L_{1}(\gamma):=\lim _{k \rightarrow+\infty}\left(E\left(\widetilde{\omega}_{\mathcal{O}^{\gamma}, k}^{\gamma}\right)+\frac{1}{2} M\left(\widetilde{\omega}_{\mathcal{O}^{\gamma}, k}^{\gamma}\right)\right)=E\left(W^{\gamma}\right)+\frac{1}{2} M\left(W^{\gamma}\right) \in\left(0, L_{\text {max }}\right],
\end{aligned}
$$


we see that

$$
\lim _{J \rightarrow+\infty}\left[\sum_{1 \leq \alpha, \beta, \gamma \leq J}\left[L_{E}(\alpha)+L_{l s}(\beta)+L_{1}(\gamma)\right]+\lim _{k \rightarrow+\infty} L\left(R_{k}^{J}\right)\right] \leq L_{\max } .
$$

The numbers $L_{E}(\alpha), L_{l s}(\beta), L_{1}(\gamma)$ and $\lim _{k \rightarrow+\infty} L\left(R_{k}^{J}\right)$ are all well defined up to taking a subsequence. Up to relabelling the profiles, we can assume that for all $\alpha, L_{\mu}(1) \geq L_{\mu}(\alpha), \mu \in\{E, l s, 1\}$.

Case IIa: $L_{E}(1)=L_{\max }$, there is only one Euclidean profile, that is

$$
u_{k}(0)=\widetilde{\varphi}_{\mathcal{E}, k}+o_{k}(1)
$$

in $H^{1}\left(\mathbb{R} \times \mathbb{T}^{2}\right)$ (see (7.15) $)$, where $\mathcal{E}$ is a Euclidean frame. In this case, since from (5.13) the corresponding nonlinear profile $U_{k}$ satisfies

$$
\left\|U_{k}\right\|_{Z(\mathbb{R})} \lesssim E_{\mathbb{R}^{3}(\varphi)} 1 \text { and } \lim _{k \rightarrow+\infty}\left\|U_{k}(0)-u_{k}(0)\right\|_{H^{1}\left(\mathbb{R} \times \mathbb{T}^{2}\right)} \rightarrow 0
$$

we may use Proposition 4.6 to deduce that

$$
\left\|u_{k}\right\|_{Z(\mathbb{R})} \lesssim\left\|u_{k}\right\|_{X^{1}(\mathbb{R})} \lesssim L_{\max } 1
$$

which contradicts (7.1).

Case IIb: $L_{l s}(1)=L_{\max }$, there is only one large scale profile, that is

$$
u_{k}(0)=\widetilde{\psi}_{\mathcal{S}, k}+o_{k}(1)
$$

in $H^{1}$ (see (7.15) $)$, where $\mathcal{S}$ is a large-scale frame. In this case, since from (5.32) the corresponding nonlinear profile $U_{k}$ satisfies

$$
\left\|U_{k}\right\|_{Z(\mathbb{R})} \lesssim_{\|\psi\|_{H^{0,1}}} 1 \quad \text { and } \quad \lim _{k \rightarrow+\infty}\left\|U_{k}(0)-u_{k}(0)\right\|_{H^{1}\left(\mathbb{R} \times \mathbb{T}^{2}\right)} \rightarrow 0
$$

we may use Proposition 4.6 to deduce that

$$
\left\|u_{k}\right\|_{Z(\mathbb{R})} \lesssim\left\|u_{k}\right\|_{X^{1}(\mathbb{R})} \lesssim L_{\max } 1
$$

which contradicts (7.1).

Case IIc: $L_{1}(1)=L_{\max }$ and, using again (7.15), we have that

$$
u_{k}(0)=\widetilde{\omega}_{\mathcal{O}, k}+o_{k}(1) \quad \text { in } H^{1}\left(\mathbb{R} \times \mathbb{T}^{2}\right),
$$

where $\mathcal{O}=\left\{\left(1, t_{k}, x_{k}, 0\right)\right\}$ is a Scale-1 frame. If $t_{k} \equiv 0$, this is precisely conclusion (17.2). If $t_{k} \rightarrow+\infty$, then, we observe that

$$
\left\|e^{i t \Delta_{\mathbb{R} \times \mathbb{T}^{2}}} \widetilde{\omega}_{\mathcal{O}_{k}}\right\|_{Z\left(a_{k}, 0\right)} \leq\left\|e^{i t \Delta_{\mathbb{R} \times \mathbb{T}^{2}}} \widetilde{\omega}_{\mathcal{O}_{k}}\right\|_{Z(-\infty, 0)}=\left\|e^{i t \Delta_{\mathbb{R} \times \mathbb{T}^{2}} \omega}\right\|_{Z\left(-\infty,-t_{k}\right)}
$$

which goes to 0 as $k \rightarrow+\infty$. Using Proposition 4.4, we see that, for $k$ large enough,

$$
\left\|u_{k}\right\|_{Z(-\infty, 0)} \leq \delta_{0}
$$

which again contradicts (7.1). The case $t_{k} \rightarrow+\infty$ is similar.

Case III: $L_{\mu}(1)<L_{\max }$ for all $\mu \in\{E, l s, 1\}$. In this case, we construct an approximate finite- $Z-$ norm solution of (1.1) with initial data $u_{k}(0)$ and derive a contradiction by invoking Proposition 4.6. Since there exists $\eta>0$ such that for all $\alpha \geq 1, \mu \in\{E, l s, 1\}, L_{\mu}(\alpha)<L_{\max }-\eta$, we have that all nonlinear profiles are global and satisfy, for any $k, \alpha \geq 1$ and $\mu \in\{E, l s, 1\}$ (after extracting a subsequence)

$$
\left\|U_{k}^{\mu, \alpha}\right\|_{Z(\mathbb{R})} \leq 2 \Lambda\left(L_{\max }-\eta / 2\right) \lesssim 1,
$$


where from now on all the implicit constants are allowed to depend on $\Lambda\left(L_{\max }-\eta / 2\right)$. Using Proposition 4.6 it follows that

$$
\left\|U_{k}^{\mu, \alpha}\right\|_{X^{1}(\mathbb{R})} \lesssim 1
$$

For $J, k \geq 1$ we define

$$
U_{\text {prof }, k}^{J}:=\sum_{\alpha=1}^{J} \sum_{\mu \in\{E, l s, 1\}} U_{k}^{\mu, \alpha} .
$$

We show first that there is a constant $Q \lesssim 1$ such that

$$
\left\|U_{\text {prof, },}^{J}\right\|_{X^{1}}^{2}+\sum_{1 \leq \alpha \leq J} \sum_{\mu \in\{E, l s, 1\}}\left\|U_{k}^{\mu, \alpha}\right\|_{X^{1}}^{2} \leq Q^{2},
$$

uniformly in $J$, for all $k$ sufficiently large. Let $\delta_{0}=\delta_{0}\left(2 L_{\max }\right)$ defined in Proposition 4.4 From (7.15), we know that there are only finitely many profiles such that $L(\alpha) \geq \delta_{0} / 2$. Without loss of generality, we may assume that for all $\alpha \geq A, L(\alpha) \leq \delta_{0}$. Using (6.14), (17.16), and (4.8) we then see that

$$
\begin{aligned}
\left\|U_{\text {prof }, k}^{J}\right\|_{X^{1}(\mathbb{R})} & =\left\|\sum_{1 \leq \alpha \leq J} \sum_{\mu \in\{E, l s, 1\}} U_{k}^{\mu, \alpha}\right\|_{X^{1}(\mathbb{R})} \\
& \leq\left\|\sum_{1 \leq \alpha \leq A} \sum_{\mu \in\{E, l s, 1\}} U_{k}^{\mu, \alpha}\right\|_{X^{1}(\mathbb{R})}+\left\|\sum_{A \leq \alpha \leq J} \sum_{\mu \in\{E, l s, 1\}}\left(U_{k}^{\mu, \alpha}-e^{i t \Delta} U_{k}^{\mu, \alpha}(0)\right)\right\|_{X^{1}(\mathbb{R})} \\
& +\left\|e^{i t \Delta} \sum_{A \leq \alpha \leq J} \sum_{\mu \in\{E, l s, 1\}} U_{k}^{\mu, \alpha}(0)\right\|_{X^{1}(\mathbb{R})} \\
& \leq \sum_{1 \leq \alpha \leq A} \sum_{\mu \in\{E, l s, 1\}}\left\|U_{k}^{\mu, \alpha}\right\|_{X^{1}(\mathbb{R})}+\sum_{A \leq \alpha \leq J} \sum_{\mu \in\{E, l s, 1\}} L_{\mu}(\alpha)^{\frac{3}{2}} \\
& +\left\|\sum_{A \leq \alpha \leq J} \sum_{\mu \in\{E, l s, 1\}} U_{k}^{\mu, \alpha}(0)\right\|_{H^{1}\left(\mathbb{R} \times \mathbb{T}^{2}\right)} \sum_{A \leq \alpha \leq J} L_{\mu \in\{E, l s, 1\}}(\alpha)^{\frac{3}{2}}+\left\|\sum_{A \leq \alpha \leq J} \sum_{\mu \in\{E, l s, 1\}} U_{k}^{\mu, \alpha}(0)\right\|_{H^{1}\left(\mathbb{R} \times \mathbb{T}^{2}\right)} \lesssim 1 .
\end{aligned}
$$

The bound on $\sum_{\alpha=1}^{J} \sum_{\mu \in\{E, l s, 1\}}\left\|U_{k}^{\mu, \alpha}\right\|_{X^{1}(\mathbb{R})}^{2}$ is similar (in fact easier), which gives (7.18).

We are now ready to construct our approximate solution. Let $F(z)=|z|^{4} z$ and introduce the notation:

$$
F^{\prime}(G) u:=3|G|^{4} u+2 G^{2}|G|^{2} \bar{u}
$$

For each $B$ and $J$, we define $g_{k}^{B, J}$ to be the solution of the initial value problem:

$$
i \partial_{t} g+\Delta g-F^{\prime}\left(U_{p r o f, k}^{B}\right) g=0, \quad g(0)=R_{k}^{J} .
$$

The solution $g_{k}^{B, J}$ is well defined on $\mathbb{R}$ for $k \geq k_{0}(J)$ and satisfies:

$$
\left\|g_{k}^{B, J}\right\|_{X^{1}(\mathbb{R})} \leq Q^{\prime} .
$$

for some constant $Q^{\prime}$ independent of $J$ and $B$. This follows by splitting $\mathbb{R}$ into $O(Q)$ intervals $I_{j}$ over which $\left\|U_{\text {prof,k }}^{B}\right\|_{Z\left(I_{j}\right)}$ is small and applying the local theory in Section 4 (in particular Lemma 4.3) on each subinterval.

We now define the approximate solution: for $A$ to be chosen shortly, we let 


$$
U_{k}^{a p p, J}=U_{\text {prof }, k}^{A}+g_{k}^{A, J}+U_{\text {prof }, k}^{>A} \quad \text { where } \quad U_{\text {prof }, k}^{>A}=\sum_{A<\alpha \leq J} \sum_{\mu \in\{E, l s, 1\}} U_{k}^{\mu, \alpha}
$$

which has $u_{k}(0)$ as its initial data and satisfies, for any $1 \leq A \leq J$, the bound:

$$
\left\|U_{k}^{a p p, J}\right\|_{X^{1}(\mathbb{R})} \leq 3\left(Q+Q^{\prime}\right)
$$

for all $k \geq k_{0}(J)$. The stability result in Proposition 4.6 with $M=6\left(1+Q+Q^{\prime}\right)$ gives us an $\epsilon_{1}=$ $\epsilon_{1}(M) \leq \frac{1}{K\left(1+Q+Q^{\prime}\right)}$ for some $K$ sufficiently large, such that if the error term defined below in (7.24) satisfies $\left\|e_{k}^{J}\right\|_{N(\mathbb{R})} \leq \epsilon_{1}$, then we can upgrade the uniform $X^{1}(\mathbb{R})$ bounds on $U_{k}^{a p p, J}$ into a uniform bound on $\left\|u_{k}\right\|_{Z(\mathbb{R})}$ thus deriving a contradiction. To do this, we first choose $A$ so that:

$$
\left\|U_{\text {prof }, k}^{>A}\right\|_{X^{1}(\mathbb{R})}^{2}+\sum_{A+1 \leq \alpha \leq J} \sum_{\mu \in\{E, l s, 1\}}\left\|U_{k}^{\mu, \alpha}\right\|_{X^{1}(\mathbb{R})}^{2} \leq \epsilon_{1}^{10} \quad \text { for any } J \geq A \text { and } k \text { sufficiently large, }
$$

which is possible by arguing as in (7.18). With $A$ fixed as above, the rest of the proof depends on Lemma 7.4 and Lemma 7.5 proved in the next subsection to bound the error term $e_{k}^{J}$ resulting from the approximate solution $U_{k}^{a p p, J}$ as follows:

$$
\begin{aligned}
e_{k}^{J}= & \left(i \partial_{t}+\Delta_{\mathbb{R} \times \mathbb{T}^{2}}\right) U_{k}^{a p p, J}-F\left(U_{k}^{a p p, J}\right) \\
= & -F\left(U_{\text {prof }, k}^{A}+g_{k}^{A, J}+U_{\text {prof }, k}^{>A}\right)+\sum_{\substack{1 \leq \alpha \leq J \\
\mu \in\{E, l s, 1\}}} F\left(U_{k}^{\mu, \alpha}\right)+F^{\prime}\left(U_{\text {prof }, k}^{A}\right) g_{k}^{A, J} \\
= & -F\left(U_{\text {prof }, k}^{A}+g_{k}^{A, J}+U_{\text {prof }, k}^{>A}\right)+F\left(U_{\text {prof }, k}^{A}+g_{k}^{A, J}\right)+F\left(U_{\text {prof }, k}^{>A}\right) \\
& -F\left(U_{\text {prof }, k}^{A}+g_{k}^{A, J}\right)+F\left(U_{\text {prof }, k}^{A}\right)+F^{\prime}\left(U_{\text {prof }, k}^{A}\right) g_{k}^{A, J} \\
& -F\left(U_{\text {prof }, k}^{A}\right)+\sum_{\substack{1 \leq \alpha \leq A \\
\mu \in\{E, l s, 1\}}} F\left(U_{k}^{\mu, \alpha}\right) \\
& -F\left(U_{\text {prof }, k}^{>A}\right)+\sum_{\substack{A+1 \leq \alpha \leq J \\
\mu \in\{E, l s, 1\}}} F\left(U_{k}^{\mu, \alpha}\right)
\end{aligned}
$$

By Lemma 4.3, (7.23), and (7.18), we estimate:

$$
\|\left(\overline{(7.26)}\left\|_{N(\mathbb{R})} \lesssim\left(\left\|U_{\text {prof }, k}^{A}+g_{k}^{A, J}\right\|_{X^{1}(\mathbb{R})}+\left\|U_{\text {prof }, k}^{>A}\right\|_{X^{1}(\mathbb{R})}\right)^{4}\right\| U_{\text {prof }, k}^{>A} \|_{X^{1}(\mathbb{R})}<\epsilon_{1} / 4\right.
$$

for $k$ large enough. By Lemma 4.3 , Lemma 7.5 we bound 13

$$
\|\left(\mathbf{7 . 2 7 )}\left\|_{N(\mathbb{R})} \lesssim\left(\left\|U_{\text {prof }, k}^{A}\right\|_{X^{1}(\mathbb{R})}+\left\|g_{k}^{A, J}\right\|_{X^{1}(\mathbb{R})}\right)^{4}\right\| g_{k}^{A, J}\left\|_{Z^{\prime}(\mathbb{R})} \lesssim\left(Q+Q^{\prime}\right)^{4}\right\| g_{k}^{A, J} \|_{Z^{\prime}(\mathbb{R})}<\epsilon_{1} / 4\right.
$$

if $J$ is sufficiently large and $k \geq k_{0}(J)$ for some $k_{0}(J)$. Turning to (7.28), by Lemma 7.4;

$$
\|\left(\overline{7.28)}\left\|_{N(\mathbb{R})} \sum_{\substack{\left(\alpha_{j}, \mu_{j}\right) \in\{1, \ldots, A\} \times\{E, l s, 1\} \\\left(\alpha_{1}, \mu_{1}\right) \neq\left(\alpha_{2}, \mu_{2}\right)}}\right\| \widetilde{U}_{k}^{\mu_{1}, \alpha_{1}} \widetilde{U}_{k}^{\mu_{2}, \alpha_{2}} \prod_{i=3}^{5} \widetilde{U}_{k}^{\mu_{i}, \alpha_{i}} \|_{N(\mathbb{R})} \leq \epsilon_{1} / 4\right.
$$

\footnotetext{
${ }^{13}$ The last term in (7.27) was introduced in (7.40) precisely to guarantee that only quadratic in $g_{k}^{A, J}$ factors appear in (7.27); otherwise one is left with terms like $\left|U^{\mu, \alpha}\right|{ }^{4} g_{k}^{A, J}$ that cannot be bounded using Lemmas 7.4 and 7.5
} 
if $k$ is large enough. Finally, by (7.23),

$$
\begin{aligned}
\|(7.29)\|_{N(\mathbb{R})} & \lesssim\left\|F\left(U_{\text {prof }, k}^{>A}\right)\right\|_{N(\mathbb{R})}+\sum_{\substack{A<\alpha \leq J \\
\mu \in\{E, l s, 1\}}}\left\|F\left(U_{\text {prof }, k}^{\mu, \alpha}\right)\right\|_{N(\mathbb{R})} \\
& \lesssim\left\|U_{\text {prof,k }}^{>A}\right\|_{X^{1}(\mathbb{R})}^{5}+\sum_{\substack{A<\alpha \leq J \\
\mu \in\{E, l s, 1\}}}\left\|U_{\text {prof,k }}^{\mu, \alpha}\right\|_{X^{1}(\mathbb{R})}^{5} \lesssim \epsilon_{1} / 4 .
\end{aligned}
$$

The upshot is that $\left\|e_{k}^{J}\right\|_{N(\mathbb{R})}<\epsilon_{1}$ if $J$ is sufficiently large and $k \geq k_{0}(J)$. Invoking the conclusion of Proposition 4.6 we get that $u_{k}$ extends as a solution in $X_{c}^{1}(\mathbb{R})$ satisfying:

$$
\left\|u_{k}\right\|_{Z(\mathbb{R})}<+\infty
$$

which is in contradiction with (7.1).

We are thus left with proving the following two lemmas:

Lemma 7.4. Assume that $U_{k}^{\alpha}, U_{k}^{\beta}, U_{k}^{\gamma_{1}}, U_{k}^{\gamma_{2}}, U_{k}^{\gamma_{3}}$ are five nonlinear profiles from the set $\left\{U_{k}^{\mu, \alpha}: 1 \leq \alpha \leq\right.$ $A, \mu \in\{E, l s, 1\}\}$ such that $U_{k}^{\alpha}$ and $U_{k}^{\beta}$ correspond to orthogonal frames. Then:

$$
\limsup _{k \rightarrow+\infty}\left\|\widetilde{U}_{k}^{\alpha} \widetilde{U}_{k}^{\beta} \prod_{i=1}^{3} \widetilde{U}_{k}^{\gamma_{i}}\right\|_{N(\mathbb{R})}=0 \quad \text { where, for } \delta \in\left\{\alpha, \beta, \gamma_{1}, \gamma_{2}, \gamma_{3}\right\}, \widetilde{U}_{k}^{\delta} \in\left\{U_{k}^{\delta}, \overline{U_{k}^{\delta}}\right\}
$$

Lemma 7.5. For any fixed $A$, it holds that:

$$
\limsup _{J \rightarrow+\infty} \limsup _{k \rightarrow+\infty}\left\|g_{k}^{A, J}\right\|_{Z(\mathbb{R})}=0
$$

7.3. Proof of Lemma 7.4. We start with some notational simplifications: We will often use the notation $U_{k}^{\alpha}, U_{k}^{\beta}$ instead of $\widetilde{U}_{k}^{\alpha}, \widetilde{U}_{k}^{\beta}$ due to the fact that all our estimates are invariant under complex conjugation of any of the arguments.

Based on the analysis in Lemma 6.2, we only need to consider non-linear profiles generated by renormalized frames. Recall that these were defined in (5.10), (5.31), (7.14) for Euclidean, large-scale, and scale-1 frames respectively. Note that any frame is equivalent to a re-normalized frame by Lemma 6.2.

Let $\theta>0$ be an arbitrary small parameter. If $\mathcal{O}^{\gamma}$ is a scale- 1 profile, then the nonlinear profile $U_{k}^{\gamma}=U_{k}^{s 1, \alpha}$ associated to the linear profile $\widetilde{\omega}_{\mathcal{O}^{\gamma}, k}^{\gamma}$ can be decomposed as follows: there exists parameters $T^{\gamma}$ and $R^{\gamma}$ depending on $\theta$ such that:

$$
\begin{aligned}
U_{k}^{s 1, \gamma}(t, x) & =\Pi_{\left(t_{k}^{\gamma}, p_{k}^{\gamma}\right)}\left[\mathbb{1}_{t \in\left[T^{\gamma},+\infty\right)} e^{i t \Delta} \phi_{+}^{\gamma}+\mathbb{1}_{t \in\left(-\infty,-T^{\gamma}\right)} e^{i t \Delta} \phi_{-}^{\gamma}+V^{\gamma}+\rho^{\gamma}\right] \\
& =\omega_{k}^{\gamma,+\infty}+\omega_{k}^{\gamma,-\infty}+\omega_{k}^{\gamma}+\rho_{k}^{\gamma} \\
& \left\|\rho^{\gamma}\right\|_{X^{1}(\mathbb{R})}+\left\|e^{i t \Delta} \phi_{ \pm}^{\gamma}\right\|_{Z^{\prime}\left( \pm\left[T^{\gamma},+\infty\right)\right)}+\left\|\left(1-P_{\geq\left(R^{\gamma}\right)^{-1}}^{x} P_{\leq R^{\gamma}}\right) V^{\gamma}\right\|_{X^{1}(\mathbb{R})} \leq \theta \\
& \left\|V^{\gamma}\right\|_{X^{1}(\mathbb{R})}+\left\|\phi_{ \pm}^{\gamma}\right\|_{H^{1}} \lesssim 1 \\
& \mid D_{x, y}^{m} V^{\gamma} \lesssim R^{\gamma} \mathbb{1}_{S_{k}^{\gamma}} \quad \text { for } 0 \leq m \leq 3 \\
& \operatorname{Supp} V^{\gamma} \subset S_{k}^{\gamma}:=\left\{(x, y, t) \in \mathbb{R} \times \mathbb{T}^{2} \times \mathbb{R}:|t| \leq T^{\gamma},|x| \leq R^{\gamma}\right\}, \quad \phi_{ \pm}^{\gamma}=P_{\leq R^{\gamma}} \phi_{ \pm}^{\gamma} .
\end{aligned}
$$


If $\mathcal{E}^{\gamma}$ is a normalized Euclidean profile, then the analysis in Section 5.1 allows us to find $R^{\gamma}$ and $T^{\gamma}$ depending on $\theta$ such that

$$
\begin{aligned}
U_{k}^{E, \gamma}(t, x) & =\Pi_{\left(t_{k}^{\gamma}, p_{k}^{\gamma}\right)}\left[\mathbb{1}_{t \in\left[\left(N_{k}^{\gamma}\right)^{-2} T^{\gamma},+\infty\right)} e^{i t \Delta} T_{N_{k}^{\gamma}}^{e} \phi_{+}^{\gamma}+\mathbb{1}_{t \in\left(-\infty,-\left(N_{k}^{\gamma}\right)^{-2} T^{\gamma}\right)} e^{i t \Delta} T_{N_{k}^{\gamma}}^{e} \phi_{-}^{\gamma}+V_{k}^{\gamma}+\rho_{k}^{\gamma}\right] \\
& =\omega_{k}^{\gamma,+\infty}+\omega_{k}^{\gamma,-\infty}+\omega_{k}^{\gamma}+\rho_{k}^{\gamma} \\
& \left\|\rho_{k}^{\gamma}\right\|_{X^{1}(\mathbb{R})}+\left\|\omega_{k}^{\gamma, \pm \infty}\right\|_{Z^{\prime}(\mathbb{R})}+\left\|\left(1-P_{\left.N_{k}^{\gamma}\left(R^{\gamma}\right)^{-1} \leq|\nabla| \leq N_{k}^{\gamma} R^{\gamma}\right)}\right) V_{k}^{\gamma}\right\|_{X^{1}(\mathbb{R})} \leq \theta \\
& \left\|V_{k}^{\gamma}\right\|_{X^{1}(\mathbb{R})}+\left\|\phi_{ \pm}^{\gamma}\right\|_{\dot{H}^{1}\left(\mathbb{R}^{3}\right)} \lesssim 1 \\
& \left|D_{x, y}^{m} V_{k}^{\gamma}\right| \lesssim R^{\gamma}\left(N_{k}^{\gamma}\right)^{1 / 2+m} \mathbb{1}_{S_{k}^{\gamma}} \quad \text { for } 0 \leq k \leq 3 \\
& \operatorname{Supp} V_{k}^{\gamma} \subset S_{k}^{\gamma}:=\left\{(x, y, t) \in \mathbb{R} \times \mathbb{T}^{2} \times \mathbb{R}:|t| \leq\left(N_{k}^{\gamma}\right)^{-2} T^{\gamma},|(x, y)| \leq\left(N_{k}^{\gamma}\right)^{-1} R^{\gamma}\right\} \\
& \phi_{ \pm}^{\gamma}=P_{\left(R^{\gamma}\right)^{-1} \leq \leq R^{\gamma}} \tilde{\phi}_{ \pm}^{\gamma}, \quad\left\|\tilde{\phi}_{ \pm}^{\gamma}\right\|_{L^{1}\left(\mathbb{R}^{3}\right)} \leq R^{\gamma} .
\end{aligned}
$$

Finally if $\mathcal{L}^{\gamma}$ is a large-scale profile, then the analysis in Section 5.2 allows us to decompose:

$$
\begin{aligned}
U_{k}^{l s, \gamma}(t, x)= & \Pi_{\left(t_{k}^{\gamma},\left(x_{k}^{\gamma}, 0\right)\right)}\left[\mathbb{1}_{t \in\left[\left(N_{k}^{\gamma}\right)^{-2} T^{\gamma},+\infty\right)} e^{i t \Delta} e^{i \xi_{k} \cdot x} T_{N_{k}^{\gamma}}^{l s} \phi_{+}^{\gamma}+\mathbb{1}_{t \in\left(-\infty,-\left(N_{k}^{\gamma}\right)^{-2} T^{\gamma}\right)} e^{i t \Delta} e^{i \xi_{k} \cdot x} T_{N_{k}^{\gamma}}^{l s} \phi_{-}^{\gamma}\right. \\
& \left.+e^{i\left(\xi_{k}, x-\left|\xi_{k}\right|^{2} t\right)} V_{k}^{\gamma}+\rho_{k}^{\gamma}\right]=\omega_{k}^{\gamma,+\infty}+\omega_{k}^{\gamma,-\infty}+\omega_{k}^{\gamma}+\rho_{k}^{\gamma} \\
& \left\|\rho_{k}^{\gamma}\right\|_{X^{1}(\mathbb{R})}+\left\|\omega_{k}^{\gamma, \pm \infty}\right\|_{Z^{\prime}(\mathbb{R})}+\left\|\left(1-P_{N_{k}^{\gamma}\left(R^{\gamma}\right)^{-1} \leq\left|\partial_{x}\right| \leq N_{k}^{\gamma} R^{\gamma}} P_{\left.\left|\nabla_{y}\right| \leq R^{\gamma}\right)}\right) V_{k}^{\gamma}\right\|_{X^{1}(\mathbb{R})} \leq \theta \\
& \left\|V_{k}^{\gamma}\right\|_{X^{1}(\mathbb{R})}+\left\|\phi_{ \pm}\right\|_{H^{0,1}\left(\mathbb{R} \times \mathbb{T}^{2}\right)} \lesssim 1 \\
& \left|D_{x}^{m} D_{y}^{k} V_{k}^{\gamma}\right| \lesssim R^{\gamma}\left(N_{k}^{\gamma}\right)^{1 / 2+m} \mathbb{1}_{S_{k}^{\gamma}} \quad \text { for } 0 \leq m, k \leq 3 \\
& \operatorname{Supp} V_{k}^{\gamma} \subset S_{k}^{\gamma}:=\left\{(x, y, t) \in \mathbb{R} \times \mathbb{T}^{2} \times \mathbb{R}:|t| \leq\left(N_{k}^{\gamma}\right)^{-2} T^{\gamma},\left|x-2 \xi_{k}^{\gamma} t\right| \leq\left(N_{k}^{\gamma}\right)^{-1} R^{\gamma}\right\} \\
& \phi_{ \pm}^{\gamma}=P_{\leq R^{\gamma}} \phi_{ \pm}^{\gamma},
\end{aligned}
$$

for some $R^{\gamma}$ and $T^{\gamma}$ depending on $\theta$.

The decomposition for Euclidean and large-scale profiles follow from Propositions 5.4 and 5.9 . The further frequency and space support properties are either consequences of the latter propositions or are obtained a posteriori by truncating and incurring an error smaller than $\theta$ in $X^{1}(\mathbb{R})$ which is absorbed in $\rho_{k}^{\gamma}$. In all three cases above, we denote by $\mathcal{S}_{k}^{\gamma}$ the translate of $S_{k}^{\gamma}$ by $\left(t_{k}^{\gamma}, p_{k}^{\gamma}\right)$, so that $\operatorname{supp} \omega_{k}^{\gamma} \subset \mathcal{S}_{k}^{\gamma}$.

Proof of Lemma 7.4. For each $\delta \in\left\{\alpha, \beta, \gamma_{1}, \gamma_{2}, \gamma_{3}\right\}$, we decompose $U_{k}^{\delta}$ as above with a smallness parameter $\theta$. Expanding the product $U_{k}^{\alpha} U_{k}^{\beta} \prod_{i=1}^{3} U_{k}^{\gamma_{i}}$ into its constituents, we directly notice that by Lemma 4.3 any monomial containing at least one factor of $\rho_{k}^{\delta}$ contributes $\lesssim \theta$ to (7.30), and similarly for any monomial with more than one factor of type $\omega_{k}^{\delta, \pm \infty}$.

There remains only two possibilities: either there are two different bulk terms $\omega_{k}^{\alpha}$ and $\omega_{k}^{\beta}$ and we can use Lemma 7.6 below, or there are four copies of the same, up to conjugation, bulk (say $\omega_{k}^{\alpha}$ ) and a linear term $\omega^{\beta, \pm \infty}$ and we can use Lemma 7.7 below. Since $\theta$ is arbitrary, we are thus only left with proving Lemmas 7.6 and 7.7 .

Lemma 7.6. With the notation as above, suppose that $f_{k}^{1}, f_{k}^{2}$, and $f_{k}^{3} \in X_{c}^{1}(\mathbb{R})$ with unit $X^{1}$ norm. For any $\theta>0$ and $1 \leq \alpha, \beta \leq A$ that correspond to orthogonal frames, there holds:

$$
\limsup _{k \rightarrow+\infty}\left\|\omega_{k}^{\alpha} \omega_{k}^{\beta} \prod_{j=1}^{3} f_{k}^{j}\right\|_{N(\mathbb{R})}=0 .
$$


Lemma 7.7. With the notation above, for any $\theta>0$, and any $1 \leq \alpha, \beta \leq A$ that correspond to orthogonal frames,

$$
\limsup _{k \rightarrow+\infty}\left\|\mathfrak{O}_{4,1}\left(\omega_{k}^{\alpha}, \omega_{k}^{\beta, \pm \infty}\right)\right\|_{N(\mathbb{R})}=0 .
$$

Recall that $\mathfrak{O}_{4,1}(a, b)$ represents an expression which is 4-linear in $\{a, \bar{a}\}$ and linear in $\{b, \bar{b}\}$.

Proof of Lemma 7.6. Recall that by Lemma 6.2 we may assume that either $N_{k}^{\alpha} / N_{k}^{\beta} \rightarrow+\infty$ or $N_{k}^{\alpha}=N_{k}^{\beta}$. In the latter case, either $\left(N_{k}^{\alpha}\right)^{2}\left|t_{k}^{\alpha}-t_{k}^{\beta}\right|+\left(N_{k}^{\alpha}\right)^{-1}\left|\xi_{k}^{\alpha}-\xi_{k}^{\beta}\right| \rightarrow+\infty$ or $t_{k}^{\alpha}=t_{k}^{\beta}$ and $\xi_{k}^{\alpha}=\xi_{k}^{\beta}$.

We fix $\kappa>0$. We start by assuming that $N_{k}^{\alpha} / N_{k}^{\beta} \rightarrow+\infty$ and let $I_{k}^{\alpha}=\left(t_{k}^{\alpha}-T^{\alpha}\left(N_{k}^{\alpha}\right)^{-2}, t_{k}^{\alpha}+T^{\alpha}\left(N_{k}^{\alpha}\right)^{-2}\right)$. Assume also that $\omega_{k}^{\beta}$ corresponds to a large scale profile or a scale-1 profile. In this case, there exists $D=D(\kappa)$ such that

$$
\left\|P_{\geq D} \omega_{k}^{\beta}\right\|_{X^{1}(\mathbb{R})} \leq \kappa
$$

and therefore,

$$
\left\|\omega_{k}^{\alpha}\left(P_{\geq D} \omega_{k}^{\beta}\right) f_{k}^{1} f_{k}^{2} f_{k}^{3}\right\|_{N\left(I_{\alpha}\right)} \leq \kappa .
$$

Independently, using (4.6) we also see that

$$
\begin{aligned}
\left\|\omega_{k}^{\alpha}\left(P_{\leq D} \omega_{k}^{\beta}\right) f_{k}^{1} f_{k}^{2} f_{k}^{3}\right\|_{N\left(I_{\alpha}\right)} & =\left\|\omega_{k}^{\alpha}\left(\mathbb{1}_{I_{k}^{\alpha}} P_{\leq D} \omega_{k}^{\beta}\right) f_{k}^{1} f_{k}^{2} f_{k}^{3}\right\|_{N\left(I_{k}^{\alpha}\right)} \lesssim_{\theta, \kappa}\left\|\mathbb{1}_{I_{k}^{\alpha}} \omega_{k}^{\beta}\right\|_{Z(\mathbb{R})} \\
& \lesssim_{\theta, \kappa} O_{k}(1)
\end{aligned}
$$

where the last line follows from the estimate in the fifth line in (7.31) and (7.33).

It remains to consider the case when both profiles are Euclidean profiles. In this case, there exists $D=D(\kappa)$ such that

$$
\left\|P_{\leq D^{-1} N_{k}^{\alpha}} \omega_{k}^{\alpha}\right\|_{X^{1}(\mathbb{R})} \lesssim \kappa \text { whereas }\left\|P_{\geq D N_{k}^{\beta}} \omega_{k}^{\beta}\right\|_{X^{1}(\mathbb{R})} \lesssim \kappa
$$

and using (7.36) and (4.6):

$$
\begin{aligned}
\left\|\omega_{k}^{\alpha} \omega_{k}^{\beta} \prod_{j=1}^{3} f_{k}^{j}\right\|_{N(\mathbb{R})} \leq & \left\|\left(P_{\leq D^{-1} N_{k}^{\alpha}} \omega_{k}^{\alpha}\right) \omega_{k}^{\beta} \prod_{j=1}^{3} f_{k}^{j}\right\|_{N(\mathbb{R})}+\left\|\left(P_{\geq D^{-1} N_{k}^{\alpha}} \omega_{k}^{\alpha}\right) \cdot\left(P_{\geq D N_{k}^{\beta}} \omega_{k}^{\beta}\right) \prod_{j=1}^{3} f_{k}^{j}\right\|_{N(\mathbb{R})} \\
& +\left\|\left(P_{\geq D^{-1} N_{k}^{\alpha}} \omega_{k}^{\alpha}\right) \cdot\left(P_{\leq D N_{k}^{\beta}} \omega_{k}^{\beta}\right) \prod_{j=1}^{3} f_{k}^{j}\right\|_{N(\mathbb{R})} \\
\leq \quad & 2 \kappa+\left\|\left(P_{\geq D^{-1} N_{k}^{\alpha}} \omega_{k}^{\alpha}\right) \cdot\left(\mathbb{1}_{I_{k}^{\alpha}} P_{\leq D N_{k}^{\beta}} \omega_{k}^{\beta}\right) \prod_{j=1}^{3} f_{k}^{j}\right\|_{N(\mathbb{R})},
\end{aligned}
$$

and once again, since for $k$ large enough, $D^{-1} N_{k}^{\alpha}>>D N_{k}^{\beta}$, using (4.6) and the fifth line in (7.32),

$$
\left\|\left(P_{\geq D^{-1} N_{k}^{\alpha}} \omega_{k}^{\alpha}\right) \cdot\left(\mathbb{1}_{I_{k}^{\alpha}} P_{\leq D N_{k}^{\beta}} \omega_{k}^{\beta}\right) \prod_{j=1}^{3} f_{k}^{j}\right\|_{N(\mathbb{R})} \lesssim_{\theta}\left\|\mathbb{1}_{I_{k}^{\alpha}} \omega_{k}^{\beta}\right\|_{Z(\mathbb{R})}=o_{k}(1) .
$$

This finishes the proof of the case $N_{k}^{\alpha} / N_{k}^{\beta} \rightarrow+\infty$.

Now assume that $N_{k}^{\alpha}=N_{k}^{\beta}$. If $N_{k}^{2}\left|t_{k}^{\alpha}-t_{k}^{\beta}\right| \rightarrow+\infty$, then by inspecting the time supports, we get that $\omega_{k}^{\alpha} \omega_{k}^{\beta}=0$ for large enough $k$. As a result, to continue we may assume $t_{k}^{\alpha} \equiv t_{k}^{\beta}$. 
Assume now that we have two large scale profiles and that $\left(N_{k}^{\alpha}\right)^{-1}\left|\xi_{k}^{\alpha}-\xi_{k}^{\beta}\right| \rightarrow+\infty$. In this case, if $(x, y, s) \in \mathcal{S}_{k}^{\alpha} \cap \mathcal{S}_{k}^{\beta}$, then

$$
\left(N_{k}^{\alpha}\right)^{-1} R^{\alpha} \geq\left|x-2 \xi_{k}^{\alpha} s\right|=\left|x-2 \xi_{k}^{\beta} s-2\left(\xi_{k}^{\alpha}-\xi_{k}^{\beta}\right) s\right| \geq N_{k}^{\alpha}|s| \cdot\left[\left(N_{k}^{\alpha}\right)^{-1}\left|\xi_{k}^{\alpha}-\xi_{k}^{\beta}\right|\right]-\left(N_{k}^{\alpha}\right)^{-1} R_{k}^{\beta} .
$$

as a result, we see that there exists an interval $I_{k}$ of length satisfying $\left(N_{k}^{\alpha}\right)^{2}\left|I_{k}\right| \rightarrow 0$ as $k \rightarrow+\infty$ such that

and using the fifth line in (17.33), we have that

$$
\omega_{k}^{\alpha} \omega_{k}^{\beta}=\left(\mathbb{1}_{I_{k}} \omega_{k}^{\alpha}\right) \cdot\left(\mathbb{1}_{I_{k}} \omega_{k}^{\beta}\right),
$$

$$
\left\|\mathbb{1}_{I_{k}} \omega_{k}^{\alpha}\right\|_{Z(\mathbb{R})}+\left\|\mathbb{1}_{I_{k}} \omega_{k}^{\beta}\right\|_{Z(\mathbb{R})} \rightarrow 0 \text { as } k \rightarrow+\infty
$$

Using Lemma 4.6, we deduce that

$$
\left\|\omega_{k}^{\alpha} \omega_{k}^{\beta} f_{k}^{1} f_{k}^{2} f_{k}^{3}\right\|_{N(\mathbb{R})} \rightarrow 0
$$

Finally, we are left with the case when $N_{k}^{\alpha} \equiv N_{k}^{\beta}, t_{k}^{\alpha} \equiv t_{k}^{\beta}$, and $\xi_{k}^{\alpha} \equiv \xi_{k}^{\beta}$, but $\left(N_{k}^{\alpha}\right)\left|x_{k}^{\alpha}-x_{k}^{\beta}\right| \rightarrow+\infty$. Here, (7.34) follows directly since $\omega_{k}^{\alpha} \omega_{k}^{\beta}=0$ for large enough $k$. Since $\kappa>0$ was arbitrary, the proof is complete.

Proof of lemma 7.7. Using that

$$
\nabla \mathfrak{O}_{4,1}\left(\omega_{k}^{\alpha}, \omega_{k}^{\beta, \pm \infty}\right)=\mathfrak{O}_{3,1,1}\left(\omega_{k}^{\alpha}, \nabla \omega_{k}^{\alpha}, \omega_{k}^{\beta, \pm \infty}\right)+\mathfrak{O}_{4,1}\left(\omega_{k}^{\alpha}, \nabla \omega_{k}^{\beta, \pm \infty}\right),
$$

we see that

$$
\begin{aligned}
& \left\|\mathfrak{O}_{4,1}\left(\omega_{k}^{\alpha}, \omega_{k}^{\beta, \pm \infty}\right)\right\|_{L_{t}^{1}\left(I_{k}^{\alpha}, H^{1}\right)} \\
& \lesssim_{\theta}\left(N_{k}^{\alpha}\right)^{\frac{1}{2}}\left[\left(1+N_{k}^{\alpha}\right)\left\|\mathfrak{O}_{1,1}\left(\omega_{k}^{\alpha}, \omega_{k}^{\beta, \pm \infty}\right)\right\|_{L_{x, y, t}^{2}\left(\mathcal{S}_{k}^{\alpha}\right)}+\left\|\mathfrak{O}_{1,1}\left(\omega_{k}^{\alpha}, \nabla \omega_{k}^{\beta, \pm \infty}\right)\right\|_{L_{x, y, t}^{2}\left(\mathcal{S}_{k}^{\alpha}\right)}\right] .
\end{aligned}
$$

where $I_{k}^{\alpha}=\left(t_{k}^{\alpha}-T^{\alpha}\left(N_{k}^{\alpha}\right)^{-2}, t_{k}^{\alpha}+T^{\alpha}\left(N_{k}^{\alpha}\right)^{-2}\right)$. Since we also have that

$$
\left\|\partial^{p} \omega_{k}^{\beta, \pm \infty}\right\|_{L_{x, y, t}^{\infty}} \lesssim_{\theta}\left(1+\left(N_{k}^{\beta}\right)^{|p|}\right)\left(N_{k}^{\beta}\right)^{\frac{1}{2}}, \quad\left\|\omega_{k}^{\alpha}\right\|_{L_{x, y, t}^{2}} \lesssim_{\theta} \begin{cases}\left(N_{k}^{\alpha}\right)^{-2} & \text { if } N_{k}^{\alpha} \geq 1 \\ \left(N_{k}^{\alpha}\right)^{-1} & \text { if } N_{k}^{\alpha} \rightarrow 0 .\end{cases}
$$

we get that when $N_{k}^{\alpha} / N_{k}^{\beta} \rightarrow+\infty$,

$$
\limsup _{k \rightarrow+\infty}\left\|\mathfrak{O}_{4,1}\left(\omega_{k}^{\alpha}, \omega_{k}^{\beta, \pm \infty}\right)\right\|_{L_{t}^{1} H_{x, y}^{1}}=0 .
$$

Assume now that $N_{k}^{\alpha} \equiv N_{k}^{\beta}$. If $\left(N_{k}^{\alpha}\right)^{2}\left|t_{k}^{\alpha}-t_{k}^{\beta}\right| \rightarrow+\infty$, we may simply use the $t^{-1 / 2}$ decay of the propagator $e^{i t \Delta}$ and replace the first bound in (7.38) by

$$
\left\|\partial^{p} \omega_{k}^{\beta, \pm \infty}\right\|_{L_{t}^{\infty}\left(I_{k}^{\alpha}: L_{x, y}^{\infty}\right)} \lesssim_{\theta}\left(1+\left(N_{k}^{\alpha}\right)^{|p|}\right)\left(N_{k}^{\alpha}\right)^{\frac{1}{2}}\left[\left(N_{k}^{\alpha}\right)^{2}\left|t_{k}^{\alpha}-t_{k}^{\beta}\right|\right]^{-1 / 2}
$$

and conclude similarly. If $t_{k}^{\alpha} \equiv t_{k}^{\beta}$ but $\left(N_{k}^{\alpha}\right)^{-1}\left|\xi_{k}^{\alpha}-\xi_{k}^{\beta}\right|+N_{k}^{\alpha}\left|\left(x_{k}^{\alpha}-x_{k}^{\beta}\right)-2\left(\xi_{k}^{\alpha}-\xi_{k}^{\beta}\right) t_{k}^{\alpha}\right| \rightarrow+\infty$, then, it is easy to see that

$$
\mathbb{1}_{\mathcal{S}_{k}^{\alpha}} \mathfrak{O}_{4,1}\left(\omega_{k}^{\alpha}, \omega_{k}^{\beta, \pm \infty}\right) \rightarrow 0 \text { in } L_{t}^{1}\left(\mathbb{R}, H_{x, y}^{1}\left(\mathbb{R} \times \mathbb{T}^{2}\right)\right) .
$$

It remains to consider the case $N_{k}^{\beta} / N_{k}^{\alpha} \rightarrow+\infty$. Fix $\kappa>0$. There exists $\delta=\delta(\kappa, \theta)>0$ such that

$$
\left\|P_{\leq \delta N_{k}^{\beta}}^{x} \omega_{k}^{\beta,+\infty}\right\|_{X^{1}(\mathbb{R})} \leq \kappa
$$

so this gives an acceptable contribution. Similar considerations hold for $(-\infty, 0)$. Independently, estimating $\omega_{k}^{\alpha}$ in $L_{x, y, t}^{\infty}$ in (7.37) and using that

$$
\left\|P_{\geq \delta N_{k}^{\beta}}^{x} \nabla \omega_{k}^{\beta, \pm \infty}\right\|_{L_{x}^{\infty} L_{y, t}^{2}} \lesssim \delta^{-1 / 2}\left(N_{k}^{\beta}\right)^{-1 / 2}
$$


which follows from:

$$
\left\|P_{\geq K} e^{i t \Delta} \phi\right\|_{L_{x}^{\infty} L_{t, y}^{2}} \lesssim K^{-1 / 2}\|\phi\|_{L^{2}\left(\mathbb{R} \times \mathbb{T}^{2}\right)}
$$

(Plancherel in $(y, t)$ and change variables, cf. (2.13) in [34]), we also find

$$
\left\|\mathfrak{O}_{4,1}\left(\omega_{k}^{\alpha}, P_{\geq \delta N_{k}^{\beta}}^{x} \omega_{k}^{\beta, \pm \infty}\right)\right\|_{L_{t}^{1}\left(I_{k}^{\alpha}, H^{1}\right)} \lesssim_{\kappa, \theta} \delta^{-3 / 2}\left(N_{k}^{\alpha} / N_{k}^{\beta}\right)^{\frac{1}{2}}
$$

Since $\kappa>0$ is arbitrary, this finishes the proof.

\subsection{Proof of Lemma 7.5,}

Proof. We would like to show that for any fixed $A$ the solution $g_{k}^{A, J}$ of:

$$
\left(i \partial_{t}+\Delta_{\mathbb{R} \times \mathbb{T}^{2}}\right) g_{k}^{J, A}-F^{\prime}\left(U_{\text {prof }, k}^{A}\right) g_{k}^{A, J}=0, \quad g_{k}^{A, J}(0)=R_{k}^{J}
$$

with $F(G) u$ being defined in (7.19) and $U_{\text {prof,k }}^{A}$ in (7.17), satisfies:

$$
\limsup _{J \rightarrow+\infty} \limsup _{k \rightarrow+\infty}\left\|g_{k}^{A, J}\right\|_{Z(\mathbb{R})}=0 .
$$

The key point here is that $R_{k}^{J}$ satisfies the following:

$$
\sup _{J, k}\left\|R_{k}^{J}\right\|_{H^{1}\left(\mathbb{R} \times \mathbb{T}^{2}\right)} \lesssim 1 \quad \text { and } \limsup _{J \rightarrow+\infty} \limsup _{k \rightarrow+\infty}\left\|e^{i t \Delta_{\mathbb{R} \times \mathbb{T}^{2}}} R_{k}^{J}\right\|_{Z(\mathbb{R})}=0 .
$$

Throughout this proof we will keep using the following claim that follows easily from a simple analysis similar to that in Proposition 4.4

Claim: Suppose that for each $1 \leq i \leq 4, u_{i}, u_{i}^{\prime}$ satisfy:

$$
\sum_{i=1}^{4}\left\|u_{i}\right\|_{X^{1}(\mathbb{R})}+\left\|u_{i}^{\prime}\right\|_{X^{1}(\mathbb{R})} \leq C, \quad\|h\|_{N(\mathbb{R})}<+\infty
$$

then for any $g_{0} \in H^{1}\left(\mathbb{R} \times \mathbb{T}^{2}\right)$, the solution $g(t)$ of the initial value problem:

$$
\left(i \partial_{t}+\Delta\right) g+\left(\prod_{i=1}^{4} u_{i}\right) g+\left(\prod_{i=1}^{4} u_{i}^{\prime}\right) \bar{g}=h, \quad g(0)=g_{0}
$$

exists for all time in $X_{c}^{1}(\mathbb{R})$ and satisfies:

$$
\|g\|_{X^{1}(\mathbb{R})} \lesssim C{ }_{C}\left\|_{H^{1}\left(\mathbb{R} \times \mathbb{T}^{2}\right)}+\right\| h \|_{N(\mathbb{R})} .
$$

As a result of this and the fact that $U_{k}^{\alpha}$ are uniformly bounded in $X_{c}^{1}(\mathbb{R})$, we obtain that $g_{k}^{A, J}$ is welldefined on $\mathbb{R}$ and satisfies:

$$
\sup _{J, k \in \mathbf{N}}\left\|g_{k}^{A, J}\right\|_{X^{1}(\mathbb{R})} \lesssim 1
$$

Let $\theta>0$ be given. We will prove the lemma by showing that the left-hand side of (7.41) is $\lesssim \sqrt{\theta}$. This will be accomplished in three steps:

Step 1: (Reduction to profile cores) With $\theta$ fixed as above, we decompose each profile $U_{k}^{\alpha}$ for $1 \leq \alpha \leq A$ as (7.31), (7.32), or (7.33) with smallness parameter $\theta$. Let $h_{k}^{J}$ be the solution of the initial value problem:

$$
\left(i \partial_{t}+\Delta_{\mathbb{R} \times \mathbb{T}^{2}}\right) h_{k}^{J}-\sum_{1 \leq \alpha \leq A} F^{\prime}\left(\omega_{k}^{\alpha}\right) h_{k}^{J}=0, \quad h(0)=R_{k}^{J} .
$$


By the above claim we have that $h_{k}^{J} \in X_{c}^{1}(\mathbb{R})$ and satisfies:

$$
\sup _{J, k}\left\|h_{k}^{J}\right\|_{X^{1}(\mathbb{R})} \lesssim 1
$$

We will reduce our problem to proving:

$$
\limsup _{J \rightarrow+\infty} \limsup _{k \rightarrow \infty}\left\|h_{k}^{J}\right\|_{Z(\mathbb{R})} \lesssim \theta .
$$

In fact, assuming (7.46), we show that:

$$
\limsup _{J \rightarrow+\infty} \limsup _{k \rightarrow+\infty}\left\|g_{k}^{A, J}-h_{k}^{J}\right\|_{X^{1}(\mathbb{R})} \lesssim \theta^{1 / 2} .
$$

To see this, define $\zeta_{k}=g_{k}^{A, J}-h_{k}^{J}$ which satisfies the following equation:

$$
\begin{aligned}
\left(i \partial_{t}+\Delta_{\mathbb{R} \times \mathbb{T}^{2}}\right) \zeta_{k}= & F^{\prime}\left(U_{\text {prof }, k}^{A}\right) \zeta_{k}+\left(F^{\prime}\left(U_{\text {prof }, k}^{A}\right) h_{k}^{J}-\sum_{1 \leq \alpha \leq A} F^{\prime}\left(U_{k}^{\alpha}\right) h_{k}^{J}\right) \\
& +\sum_{1 \leq \alpha \leq A}\left(F^{\prime}\left(U_{k}^{\alpha}\right) h_{k}^{J}-F^{\prime}\left(\omega_{k}^{\alpha}\right) h_{k}^{J}\right)
\end{aligned}
$$

Estimate (7.47) would follow directly (for $k$ large enough) using (7.43) once we show that:

$$
\begin{aligned}
& \limsup _{k \rightarrow+\infty}\left\|F^{\prime}\left(U_{\text {prof, }, k}^{A}\right) h_{k}^{J}-\sum_{1 \leq \alpha \leq A} F^{\prime}\left(U_{k}^{\alpha}\right) h_{k}^{J}\right\|_{N(\mathbb{R})} \lesssim \theta \\
& \limsup _{J \rightarrow+\infty} \limsup _{k \rightarrow+\infty}\left\|\sum_{1 \leq \alpha \leq A}\left(F^{\prime}\left(U_{k}^{\alpha}\right) h_{k}^{J}-F^{\prime}\left(\omega_{k}^{\alpha}\right) h_{k}^{J}\right)\right\|_{N(\mathbb{R})} \lesssim \theta .
\end{aligned}
$$

To prove (17.48), expand

$$
F^{\prime}\left(U_{p r o f, k}^{A}\right) h_{k}^{J}-\sum_{1 \leq \alpha \leq A} F^{\prime}\left(U_{k}^{\alpha}\right) h_{k}^{J}=\sum_{1 \leq \alpha, \beta, \gamma, \delta \leq A} \tilde{U}_{k}^{\alpha} \tilde{U}_{k}^{\beta} \tilde{U}_{k}^{\gamma} \tilde{U}_{k}^{\delta} h_{k}^{J},
$$

where the first two profiles differ by more than complex conjugation, then expand each profile as in (7.31), (7.32), (7.33) with smallness parameter $\theta$. The term involving $\omega_{k}^{\alpha} \omega_{k}^{\beta}$ is then controlled using Lemma 7.6, while the bound for the other terms follows from (4.7) since there are at least two terms which are small in $Z^{\prime}$. Similarly, (7.49) follows from (4.7) and the $Z^{\prime}$-norm smallness of the asymptotic components $\omega_{k}^{\alpha, \pm \infty}$ which give:

$$
\sum_{1 \leq \alpha \leq A}\left\|F^{\prime}\left(U_{k}^{\alpha}\right) h_{k}^{J}-F^{\prime}\left(\omega_{k}^{\alpha}\right) h_{k}^{J}\right\|_{N(\mathbb{R})} \lesssim \theta+\left\|h_{k}^{J}\right\|_{Z^{\prime}(\mathbb{R})}
$$

which is $\lesssim \theta^{1 / 2}$ once we prove (7.46).

\section{Step 2 (Contribution of low frequencies and high-frequency smoothing directions)}

Let $\kappa>0$ be a small dyadic number to be chosen later in terms of $\theta$. We will decompose $R_{k}^{J}$ into three components: a low frequency part, a high frequency smoothing part, and a high frequency non-smoothing part:

$$
R_{k}^{J}=P_{\leq \kappa^{-1}} R_{k}^{J}+\widetilde{P}_{\kappa}^{1} P_{>\kappa^{-1}} R_{k}^{J}+\left(1-\widetilde{P}_{\kappa}^{1}\right) P_{>\kappa^{-1}} R_{k}^{J}
$$

where $\widetilde{P}_{\kappa}^{1}$ was defined in (2.1). In this step we will estimate the contribution of first two terms above: Let $\sigma_{k}^{J, \kappa}$ be the solution to the initial value problem: 


$$
\left(i \partial_{t}+\Delta_{\mathbb{R} \times \mathbb{T}^{2}}\right) \sigma_{k}^{J, \kappa}+\sum_{1 \leq \alpha \leq A} F^{\prime}\left(\omega_{k}^{\alpha}\right) \sigma_{k}^{J, \kappa}=0, \quad \sigma_{k}^{J, \kappa}(0)=R_{k}^{J, 0}:=P_{\leq \kappa^{-1}} R_{k}^{J}+\widetilde{P}_{\kappa}^{1} P_{>\kappa^{-1}} R_{k}^{J} .
$$

As in (7.43), we have that $\sigma_{k}^{J, \kappa}$ is well-defined and satisfies $\left\|\sigma_{k}^{J, \kappa}\right\|_{X^{1}(\mathbb{R})} \lesssim 1$. Applying (7.43) to the difference equation between (17.50) and the linear homogeneous Schrödinger equation, we get:

$$
\left\|\sigma_{k}^{J, \kappa}-e^{i t \Delta} R_{k}^{J, 0}\right\|_{X^{1}(\mathbb{R})} \lesssim \sum_{1 \leq \alpha \leq A}\left\|F^{\prime}\left(\omega_{k}^{\alpha}\right) e^{i t \Delta}\left(P_{\leq \kappa^{-1}\left\langle N_{k}^{\alpha}\right\rangle} R_{k}^{J, 0}+P_{>\kappa^{-1}\left\langle N_{k}^{\alpha}\right\rangle} R_{k}^{J, 0}\right)\right\|_{N(\mathbb{R})} .
$$

Using (4.6), we directly see that for any $1 \leq \alpha \leq A$,

$$
\begin{aligned}
\limsup _{J \rightarrow+\infty} \limsup _{k \rightarrow+\infty}\left\|F^{\prime}\left(\omega_{k}^{\alpha}\right) e^{i t \Delta} P_{\leq \kappa^{-1}\left\langle N_{k}^{\alpha}\right\rangle} R_{k}^{J, 0}\right\|_{N(\mathbb{R})} & \lesssim \limsup _{J \rightarrow+\infty} \limsup _{k \rightarrow+\infty}\left\|e^{i t \Delta} P_{\leq \kappa^{-1}\left\langle N_{k}^{\alpha}\right\rangle} R_{k}^{J, 0}\right\|_{Z^{\prime}(\mathbb{R})} \\
& =0 .
\end{aligned}
$$

We now bound the contribution of $P_{>\kappa^{-1}\left\langle N_{k}^{\alpha}\right\rangle} R_{k}^{J, 0}=\widetilde{P}_{\kappa}^{1} P_{>\kappa^{-1}\left\langle N_{k}^{\alpha}\right\rangle} R_{k}^{J}$ : Using the convention that $\nabla^{1} h:=$ $|h|+|\nabla h|$, we estimate:

$$
\begin{aligned}
&\left\|F^{\prime}\left(\omega_{k}^{\alpha}\right) e^{i t \Delta} P_{>\kappa^{-1}\left\langle N_{k}^{\alpha}\right\rangle} R_{k}^{J, 0}\right\|_{N(\mathbb{R})} \lesssim\left\|\left(\omega_{k}^{\alpha}\right)^{4} \nabla^{1}\left(e^{i t \Delta} P_{>\kappa^{-1}\left\langle N_{k}^{\alpha}\right\rangle} R_{k}^{J, 0}\right)\right\|_{L_{t}^{1} L_{x, y}^{2}} \\
&+\left\|\left(\omega_{k}^{\alpha}\right)^{3} \nabla \omega_{k}^{\alpha} e^{i t \Delta} P_{>\kappa^{-1}\left\langle N_{k}^{\alpha}\right\rangle} R_{k}^{J, 0}\right\|_{L_{t}^{1} L_{x, y}^{2}} \\
& \lesssim\left\|\left(\omega_{k}^{\alpha}\right)^{4}\right\|_{L_{t}^{2} L_{x, y}^{\infty}}\left\|\mathbb{1}_{\mathcal{S}_{k}^{\alpha}} \nabla^{1}\left(e^{i t \Delta} P_{\kappa}^{1} P_{>\kappa^{-1}\left\langle N_{k}^{\alpha}\right\rangle} R_{k}^{J}\right)\right\|_{L_{x, y, t}^{2}\left(\mathcal{S}_{k}^{\alpha}\right)} \\
&+\kappa\left\langle N_{k}^{\alpha}\right\rangle^{-1}\left\|\left(\omega_{k}^{\alpha}\right)^{3} \nabla \omega_{k}^{\alpha}\right\|_{L_{t}^{2} L_{x, y}^{\infty}}\left\|\nabla^{1}\left(e^{i t \Delta} P_{\kappa}^{1} P_{>\kappa^{-1}\left\langle N_{k}^{\alpha}\right\rangle} R_{k}^{J}\right)\right\|_{L_{x, y, t}^{2}\left(\mathcal{S}_{k}^{\alpha}\right)} \\
& \lesssim{ }_{\theta} N_{k}^{\alpha} \| \mathbb{1}_{\mathcal{S}_{k}^{\alpha}} \nabla^{1}\left(e^{i t \Delta} P_{\kappa}^{1} P_{>\kappa}{ }^{-1}\left\langle N_{k}^{\alpha}\right\rangle\right. \\
&\left.R_{k}^{J}\right) \|_{L_{x, y, t}^{2}} \lesssim_{\theta} \kappa^{\frac{1}{100}},
\end{aligned}
$$

which follows from Lemma 7.9 at the end of this section. Using again (7.42) we conclude after combining (7.51), (7.52) and (7.53) that, whenever $\kappa \leq \kappa_{1}(\theta)$,

$$
\left\|\sigma_{k}^{J, \kappa}\right\|_{Z(\mathbb{R})} \leq C(\theta, A) \kappa^{\frac{1}{100}} \leq \theta .
$$

Step 3: (Contribution of high-frequency non-smoothing directions) Let $f_{k}^{J, \kappa}$ denote the solution of the initial value problem:

$$
\left(i \partial_{t}+\Delta_{\mathbb{R} \times \mathbb{T}^{2}}\right) f_{k}^{J, \kappa}-\sum_{\alpha=1}^{A} F^{\prime}\left(\omega_{k}^{\alpha}\right) f_{k}^{J, \kappa}=0, \quad f_{k}^{J, \kappa}(0)=R_{k}^{J, 1}:=\left(1-\widetilde{P}_{\kappa}^{1}\right) P_{\geq \kappa^{-1}} R_{k}^{J}
$$

We would like to show that if $k$ is large enough and $J \geq A$ :

$$
\left\|f_{k}^{J, \kappa}\right\|_{Z(\mathbb{R})} \lesssim \theta
$$

whenever $\kappa \leq \kappa_{2}(\theta)$. This combined with (17.54) and the fact that $h_{k}^{J}=\sigma_{k}^{J, \kappa}+f_{k}^{J, \kappa}$ gives (7.46) thus finishing the proof.

The idea behind the proof of (7.56) is to exploit the narrowness of the angular aperture of the Fourier support of the initial data $R_{k}^{J, 1}$ represented by the smallness of $\kappa$. The key observation is that the smallness of $\kappa$ implies that of $\left\|e^{i t \Delta} R_{k}^{J, 1}\right\|_{Z(\mathbb{R})}$, which essentially follows from (3.24). Translating this information from the homogeneous linear Schrödinger equation to the inhomogeneous one (7.55) is done via a slightly delicate perturbative argument using an appropriately modified $X^{1}$-type norm that-unlike $X^{1}$ - captures the gain reflected in (3.24). We now move to the details: 
For dyadic numbers $1 \leq M \leq N$, we define:

$$
\begin{aligned}
& p_{N, M}(\xi)=\left[\eta^{3}(\xi / 2 N)-\eta^{3}(\xi / N)\right]\left[\eta^{1}\left(\xi_{1} / 2 M\right)-\eta^{1}\left(\xi_{1} / M\right)\right] \quad \text { if } M \geq 2 \\
& p_{N, 1}(\xi)=\left[\eta^{3}(\xi / N)-\eta^{3}(\xi / 2 N)\right] \eta^{1}\left(\xi_{1} / 2\right)
\end{aligned}
$$

and the corresponding operators:

$$
\mathcal{F} P_{N, M} f=p_{N, M} \mathcal{F} f, \quad P_{N, \leq K}=\sum_{1 \leq M \leq \min (K, N)} P_{N, M}
$$

Given $\rho \in(0,1]$, we define the weights:

$$
c_{\rho, N, M}:=\left[M / N+\rho+(1+\rho N)^{-1}\right]^{\delta_{0} / 100}, \quad c_{\rho, N, M} \simeq \begin{cases}1 & \text { if } N \leq \rho^{-1} \\ \max (M / N, 1 /(N \rho))^{\frac{\delta_{0}}{100}} & \text { if } \rho^{-1} \leq N \leq \rho^{-2} \\ \max (M / N, \rho)^{\frac{\delta_{0}}{100}} & \text { if } N \geq \rho^{-2}\end{cases}
$$

where $\delta_{0}$ is the same constant as in (4.4). For functions $f \in X^{1}(I)$ on intervals $I \subset \mathbb{R}$ and $g \in H^{1}\left(\mathbb{R} \times \mathbb{T}^{2}\right)$, we defined the norms:

$$
\begin{aligned}
\|f\|_{\widetilde{X}_{\rho}^{1}(I)}^{2} & :=\sum_{N \geq 1} \sum_{1 \leq M \leq N} c_{\rho, N, M}^{2}\left\|P_{N, M} f\right\|_{X^{1}(I)}^{2} \\
\|g\|_{\widetilde{H}_{\rho}^{1}\left(\mathbb{R} \times \mathbb{T}^{2}\right)}^{2} & :=\sum_{N \geq 1} \sum_{1 \leq M \leq N} c_{\rho, N, M}^{2}\left\|P_{N, M} g\right\|_{H^{1}\left(\mathbb{R} \times \mathbb{T}^{2}\right)}^{2}
\end{aligned}
$$

From (7.57), we see that

$$
\begin{aligned}
\rho^{\delta_{0} / 100}\|f\|_{X^{1}(I)} & \lesssim\|f\|_{\widetilde{X}_{\rho}^{1}(I)} \lesssim\|f\|_{X^{1}(I)} \\
\rho^{\delta_{0} / 100}\|g\|_{H^{1}\left(\mathbb{R} \times \mathbb{T}^{2}\right)} & \lesssim\|g\|_{\widetilde{H}_{\rho}^{1}\left(\mathbb{R} \times \mathbb{T}^{2}\right)} \lesssim\|g\|_{H^{1}\left(\mathbb{R} \times \mathbb{T}^{2}\right)}
\end{aligned}
$$

It is obvious that the $\widetilde{X}_{\rho}^{1}$ satisfies:

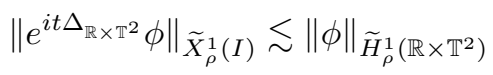

for any interval $I$, from which follows the smallness of $\left\|e^{i t \Delta}\left(1-\tilde{P}_{\kappa}^{1}\right) P_{\geq \kappa^{-1}} \phi\right\|_{\widetilde{X}_{\rho}^{1}}$ for any $\kappa \leq \rho$. This will be particularly useful since $\widetilde{X}_{\rho}^{1}$ controls the $Z$ norm: for any $\rho \in(0,1]$ :

$$
\|f\|_{Z(I)} \lesssim\|f\|_{\widetilde{X}_{\rho}^{1}(I)}
$$

In fact, (3.24) and Lemma 4.1 imply that for $p_{0} \in\left\{\frac{9}{2}, 18\right\}$ :

$$
N^{\frac{5}{p_{0}}-\frac{1}{2}}\left\|P_{N, M} f\right\|_{l_{\gamma}^{\frac{4 p_{0}}{p^{-2}}} L_{x, y, t}^{p_{0}}\left(\mathbb{R} \times \mathbb{T}^{2} \times I\right)} \lesssim\left(\frac{M}{M+N}\right)^{1 / 20} N\left\|P_{N, M} f\right\|_{U_{\Delta}^{9 / 2}(I)},
$$

which gives:

$$
\begin{aligned}
\|f\|_{Z(I)} & \leq \sum_{p_{0} \in\{9 / 2,18\}}\left(\sum_{N \geq 1} N^{\frac{10}{p_{0}}-1}\left[\sum_{1 \leq M \leq N}\left\|P_{N, M} f\right\|_{l_{\gamma}^{\frac{4 p_{0}}{p^{-2}}} L_{x, y, t}^{p_{0}}\left(\mathbb{R} \times \mathbb{T}^{2} \times I\right)}\right]^{2}\right)^{1 / 2} \\
& \lesssim\left(\sum_{N \geq 1}\left[\sum_{1 \leq M \leq N}\left(\frac{M}{N}\right)^{1 / 20}\left\|P_{N, M} f\right\|_{X^{1}(I)}\right]^{1 / 2} \lesssim\left(\sum_{N \geq 1} \sum_{1 \leq M \leq N}\left(\frac{M}{N}\right)^{1 / 20}\left\|P_{N, M} f\right\|_{X^{1}(I)}^{2}\right)^{1 / 2}\right. \\
& \lesssim\|f\|_{\widetilde{X}_{\rho}^{1}(I)}
\end{aligned}
$$


which proves (7.60). We will prove the following lemma:

Lemma 7.8. Let $D \geq 1$ be dyadic, $\theta \in(0,1]$, and $f_{k} \in X_{c}^{1}(\mathbb{R})$ be a solution to the linear equation:

$$
\left(i \partial_{t}+\Delta_{\mathbb{R} \times \mathbb{T}^{2}}\right) f_{k}-\sum_{1 \leq \alpha \leq A} F^{\prime}\left(\omega_{k, D}^{\alpha}\right) f_{k}=0
$$

where $\omega_{k, D}^{\alpha}$ is $P_{\leq D} \omega_{k}^{\alpha}$ if $N_{k}^{\alpha} \leq 1$ and $\left(P_{\leq D N_{k}^{\alpha}}-P_{\geq D^{-1} N_{k}^{\alpha}}\right) \omega_{k}^{\alpha}$ if $N_{k}^{\alpha} \rightarrow+\infty$. Then there exists $\rho_{0}=$ $\rho_{0}(\theta, D)$ such that for all $\rho \leq \rho_{0}$ and $k \geq k(\rho)$ sufficiently large, it holds that:

$$
\left\|f_{k}\right\|_{\widetilde{X}_{\rho}^{1}(\mathbb{R})} \lesssim D, \theta\left\|f_{k}(0)\right\|_{\widetilde{H}_{\rho}^{1}\left(\mathbb{R} \times \mathbb{T}^{2}\right)} \cdot
$$

With this lemma in hand, we can finish the proof as follows: by the decompositions in (7.31), (7.32), and (7.33), there exists a dyadic $D \geq 1$ such that $\left\|\omega_{k}^{\alpha}-\omega_{k, D}^{\alpha}\right\|_{X^{1}(\mathbb{R})} \leq \theta$. Denoting by $\tilde{f}_{k}^{J, \kappa}$ the solution to the initial value problem:

$$
\left(i \partial_{t}+\Delta_{\mathbb{R} \times \mathbb{T}^{2}}\right) \tilde{f}_{k}^{J, \kappa}-F^{\prime}\left(\omega_{k, D}^{\alpha}\right) \tilde{f}_{k}^{J, \kappa}=0, \quad \widetilde{f}_{k}^{J, \kappa}(0)=R_{k}^{J, 1}:=\left(1-\widetilde{P}_{\kappa}^{1}\right) P_{\geq \kappa^{-1}} R_{k}^{J}
$$

It follows from (7.43) that:

$$
\left\|f_{k}^{J, \kappa}-\tilde{f}_{k}^{J, \kappa}\right\|_{X^{1}(\mathbb{R})} \lesssim \theta
$$

for $k$ large enough, whereas it follows from Lemma 7.8 and (7.60) above that for $\rho \leq \rho(\theta, D)$ :

$$
\left\|\tilde{f}_{k}^{J, \kappa}\right\|_{Z(\mathbb{R})} \lesssim\left\|\widetilde{f}_{k}^{J, \kappa}\right\|_{\widetilde{X}_{\rho}^{1}(\mathbb{R})} \lesssim D, \theta\left\|R_{k}^{J, 1}\right\|_{\widetilde{H}_{\rho}^{1}}
$$

for $k$ large enough. Choosing $\kappa=\rho^{100}$, we have that $\left\|R_{k}^{J, 1}\right\|_{\widetilde{H}_{\rho}^{1}} \lesssim \kappa^{\delta_{0}}$ and (7.56) now follows by combining the above two inequalities. We are left with proving Lemma 7.8

7.5. Proof of Lemma 7.8, As before, we denote for any interval $I \subset \mathbb{R}$ :

$$
\|f\|_{\widetilde{N}_{\rho}(I)}=\left\|\int_{0}^{t} e^{i(t-s) \Delta} f(s) d s\right\|_{\widetilde{X}_{\rho}^{1}(I)} .
$$

Using the time-divisibility of the $Z$-norm and the nature of the decomposition in (7.31), (7.32), and (7.33), we can split $\mathbb{R}$ for each $\mu>0$ into $O_{\theta, \mu}(1)$ intervals $I$ for which:

$$
\sum_{1 \leq \alpha \leq A}\left\|\omega_{k, D}^{\alpha}\right\|_{Z^{\prime}(I)} \leq \mu
$$

We will eventually choose $\mu=\mu(\theta, D, A, Q)$ independent of $\rho, \kappa$, which means that it would be enough to prove that the analogue of (7.62) for each interval $I$ (with $f_{k}(0)$ replaced by the initial value of $f_{k}$ on that interval). By (7.43), this would follow if we prove that for each interval $I$ and for every $1 \leq \alpha \leq A$,

$$
\left\|F^{\prime}\left(\omega_{k, D}^{\alpha}\right) f\right\|_{\widetilde{N}_{\rho}(I)} \leq \frac{1}{2 A}\|f\|_{\widetilde{X}_{\rho}^{1}}
$$

if $k \geq k(\rho)$ is large enough. To prove this we will need the following refinement of (4.4): suppose that $1 \leq M_{1} \leq N_{1}, 1 \leq M_{2} \leq N_{2} \leq N_{1}$, and $N_{3} \leq N_{2}$ are all dyadic. Then:

$$
\begin{aligned}
& \left\|P_{N_{1}, M_{1}} u_{1} P_{N_{2}, \leq M_{2}} u_{2} P_{N_{3}} u_{3}\right\|_{L_{x, y, t}^{2}\left(\mathbb{R} \times \mathbb{T}^{2} \times I\right)} \\
& \lesssim\left[\left(\frac{N_{2}}{N_{1}}+\frac{1}{N_{2}}\right) \frac{M_{2}}{N_{2}} \frac{M_{1}}{M_{1}+N_{2}}\right]^{\delta_{0} / 2}\left\|P_{N_{1}, M_{1}} u_{1}\right\|_{Y^{0}(I)}\left(N_{2}\left\|P_{N_{2}, \leq M_{2}} u_{2}\right\|_{Y^{0}(I)}\right)\left(N_{3}\left\|P_{N_{3}} u_{3}\right\|_{Y^{0}(I)}\right) .
\end{aligned}
$$


This follows by interpolating (4.4) and the following refinement of (4.5) obtained by using (7.61): if $C$ is any cube in $\mathbb{R}^{3}$ with side-length $\sim N_{2}$ :

$$
\begin{aligned}
& \left\|P_{C} P_{N_{1}, M_{1}} u_{1} P_{N_{2}, \leq M_{2}} u_{2} P_{N_{3}} u_{3}\right\|_{L_{x, y, t}^{2}\left(\mathbb{R} \times \mathbb{T}^{2} \times I\right)} \\
& \lesssim\left\|P_{C} P_{N_{1}, M_{1}} u_{1}\right\|_{l_{\gamma}^{\frac{36}{5}} L_{x, y, t}^{\frac{9}{2}}}\left\|P_{N_{2}, \leq M_{2}} u_{2}\right\|_{l_{\gamma}^{\frac{36}{5}} L_{x, y, t}^{9 / 2}}\left\|P_{N_{3}} u_{3}\right\|_{l_{\gamma}^{\frac{9}{2}} L_{x, y, t}^{18}} \\
& \lesssim N_{2}^{\frac{7}{18}}\left(\frac{M_{1}}{M_{1}+N_{2}}\right)^{1 / 20}\left\|P_{C} P_{N_{1}, M_{1}} u_{1}\right\|_{Y^{0}}\left\|P_{N_{2}, \leq M_{2}} u_{2}\right\|_{l_{\gamma}^{\frac{36}{5}} L_{x, y, t}^{9 / 2}}\left\|P_{N_{3}} u_{3}\right\|_{l_{\gamma}^{\frac{9}{2}} L_{x, y, t}^{18}} \\
& \lesssim N_{2} N_{3}\left(\frac{M_{2}}{N_{2}} \frac{M_{1}}{M_{1}+N_{2}}\right)^{1 / 20}\left\|P_{C} u_{1}\right\|_{Y^{0}}\left\|u_{2}\right\|_{Y^{0}}\left\|u_{3}\right\|_{Y^{0}} \text {. }
\end{aligned}
$$

To continue we split into two cases:

Case 1: $N_{k} \leq 1$ for all $k$. In this case, we have that $\omega_{k, D}^{\alpha}=P_{\leq D} \omega_{k}^{\alpha}$. Writing $f=P_{\leq \rho^{-1}} f+P_{\geq \rho^{-1}} f$, we estimate using (4.6):

$$
\left\|F^{\prime}\left(\omega_{k, D}^{\alpha}\right) P_{\leq \rho^{-1}} f\right\|_{N(I)} \lesssim \mu^{3}\left\|P_{\leq \rho^{-1}} f\right\|_{X^{1}(I)} \lesssim \mu^{3}\|f\|_{\widetilde{X}_{\rho}^{1}(I)}
$$

since $c_{\rho, N, M} \sim 1$ if $N \lesssim \rho^{-1}$. On the other hand, for $N \geq \rho^{-1}$,

$$
P_{N, M}\left[F^{\prime}\left(\omega_{k, D}^{\alpha}\right) P_{\geq \rho^{-1}} f\right]=\sum_{N^{\prime} \sim_{D} N, M^{\prime} \sim_{D} M} P_{N, M}\left[F^{\prime}\left(\omega_{k, D}^{\alpha}\right) P_{N^{\prime}, M^{\prime}} P_{\geq \rho^{-1}} f\right]
$$

and as a result, we can estimate:

$$
\begin{aligned}
\left\|F^{\prime}\left(\omega_{k, D}^{\alpha}\right) P_{\geq \rho^{-1}} f\right\|_{\widetilde{N}_{\rho}(I)}^{2} & \lesssim \sum_{N, M} c_{\rho, N, M}^{2}\left\|P_{N, M}\left[F^{\prime}\left(\omega_{k, D}^{\alpha}\right) P_{\geq \rho^{-1}} f\right]\right\|_{N(I)}^{2} \\
& \lesssim \sum_{N, M} \sum_{N^{\prime} \sim_{D}, M^{\prime} \sim_{D} M} c_{\rho, N, M}^{2}\left\|F^{\prime}\left(\omega_{k, D}^{\alpha}\right) P_{N^{\prime}, M^{\prime}} P_{\geq \rho^{-1}} f\right\|_{N(I)}^{2} \\
& \lesssim \sum_{N, M N^{\prime} \sim_{D} N, M^{\prime} \sim_{D} M} \sum_{\rho, N^{\prime}, M^{\prime}}^{2} \mu^{3}\left\|P_{N^{\prime}, M^{\prime}} P_{\geq \rho^{-1}} f\right\|_{X^{1}(I)}^{2} \lesssim_{D} \mu^{3}\|f\|_{\widetilde{X}_{\rho}^{1}(I)}^{2},
\end{aligned}
$$

which gives (7.64) in this case provided $\mu$ is chosen small enough depending on $A, D, \theta$.

Case 2: $N_{k} \rightarrow+\infty$ : The analysis in this case is more delicate. We will assume throughout that $N_{k}^{\alpha} \geq \rho^{-2}$. The contribution of low frequencies $P_{\leq 2^{10} D N_{k}^{\alpha}}$ is easily estimated using (4.6) as follows:

$$
\left\|F^{\prime}\left(\omega_{k, D}\right) P_{\leq 2^{10} D N_{k}^{\alpha}} f\right\|_{N(I)} \lesssim_{D} \mu^{3}\left\|P_{\leq 2^{10} D N_{k}^{\alpha}} f\right\|_{Z(I)} \lesssim \mu^{3}\|f\|_{\widetilde{X}_{\rho}^{1}(I)} .
$$

We now turn to the contribution of $P_{\geq 2^{10} D N_{k}^{\alpha}} f$ : by inspecting the Fourier supports, we notice that:

$$
P_{N, M}\left[F^{\prime}\left(\omega_{k, D}^{\alpha}\right) P_{\geq 2^{10} D N_{k}^{\alpha}} f\right]=\sum_{N^{\prime} \sim N, M^{\prime} \sim M} P_{N, M}\left[F^{\prime}\left(\omega_{k, D}^{\alpha}\right) P_{N^{\prime}, M^{\prime}} P_{\geq 2^{10} D N_{k}^{\alpha}} f\right]
$$

if $M \geq 2^{5} D N_{k}^{\alpha}$, and

$$
P_{N, \leq 2^{5} D N_{k}^{\alpha}}\left[F^{\prime}\left(\omega_{k, D}^{\alpha}\right) P_{\geq 2^{10} D N_{k}^{\alpha}} f\right]=\sum_{N^{\prime} \sim N, M^{\prime} \leq 2^{5} D N_{k}^{\alpha}} P_{N, \leq 2^{5} D N_{k}^{\alpha}}\left[F^{\prime}\left(\omega_{k, D}^{\alpha}\right) P_{N^{\prime}, M^{\prime}} P_{\geq 2^{10} D N_{k}^{\alpha}} f\right] .
$$

In the case when $M \geq 2^{5} D N_{k}^{\alpha}$, we can proceed as in Case 1 to get:

$$
\left\|\sum_{N \geq 1} \sum_{M>2^{5} D N_{k}^{\alpha}} P_{N, M}\left[F^{\prime}\left(\omega_{k, D}^{\alpha}\right) P_{\geq 2^{10} D N_{k}^{\alpha}} f\right]\right\|_{\tilde{N}_{\rho}(I)} \lesssim \mu^{3}\|f\|_{\tilde{X}_{\rho}^{1}(I)}
$$


Moving on to the case when $M \leq 2^{5} D N_{k}^{\alpha}$, we first denote $\tilde{f} \in\left\{P_{\geq 2^{10} D N_{k}^{\alpha}} f, \overline{P_{\geq 2^{10} D N_{k}^{\alpha} f}}\right\}, \widetilde{\omega}_{k, D}^{\alpha} \in$ $\left\{\omega_{k, D}^{\alpha}, \overline{\omega_{k, D}^{\alpha}}\right\}$ and estimate, for $N^{\prime} \geq 2^{5} D N_{k}^{\alpha}, M^{\prime} \leq 2^{5} D N_{k}^{\alpha}$, using Proposition 2.1, and (7.65):

$$
\begin{aligned}
\left\|\mathcal{O}_{4,1}\left(\omega_{k, D}^{\alpha}, P_{N^{\prime}, M^{\prime}} f\right)\right\|_{N(I)} & =\sup _{\|h\|_{Y^{-1}(I)}=1}\left|\int_{\mathbb{R} \times \mathbb{T}^{2} \times I} h \cdot \mathfrak{O}_{4,1}\left(\omega_{k, D}^{\alpha}, P_{N^{\prime}, M^{\prime}} f\right) d x d y d t\right| \\
& \lesssim\left\|\mathfrak{O}_{2,1}\left(\omega_{k, D}^{\alpha}, P_{N^{\prime}, M^{\prime}} f\right)\right\|_{L_{x, y, t}^{2}{ }_{\|h\|_{Y-1}(I)}=1}\left\|\mathfrak{O}_{2,1}\left(\omega_{k}^{\alpha},\left(P_{\leq 2 N^{\prime}}-P_{\leq N^{\prime} / 2}\right) h\right)\right\|_{L_{x, y, t}^{2}} \\
& \lesssim{ }_{D, \theta} \mu^{4}\left[\left(\frac{N_{k}^{\alpha}}{N^{\prime}}+\frac{1}{N_{k}^{\alpha}}\right) \frac{M^{\prime}}{M^{\prime}+N_{k}^{\alpha}}\right]^{\delta_{0} / 2}\left\|P_{N^{\prime}, M^{\prime}} \tilde{f}\right\|_{Y^{0}(I)} .
\end{aligned}
$$

Note that 14 :

$$
\begin{aligned}
{\left[\left(\frac{N_{k}^{\alpha}}{N^{\prime}}+\frac{1}{N_{k}^{\alpha}}\right) \frac{M^{\prime}}{M^{\prime}+N_{k}^{\alpha}}\right]^{\delta_{0} / 4} } & \lesssim\left(\frac{M^{\prime}}{N^{\prime}}\right)^{\delta_{0} / 4} \lesssim c_{\rho, N^{\prime}, M^{\prime}} & \text { if } N^{\prime} \leq\left(N_{k}^{\alpha}\right)^{2} \\
& \lesssim\left(\frac{M^{\prime}}{\left(N_{k}^{\alpha}\right)^{2}}\right)^{\delta_{0} / 4} \lesssim_{D} \rho^{\delta_{0} / 2} \lesssim c_{\rho, N^{\prime}, M^{\prime}} & \text { if } N^{\prime} \geq\left(N_{k}^{\alpha}\right)^{2} \geq \rho^{-4} .
\end{aligned}
$$

As a result we get that:

$$
\sum_{1 \leq M^{\prime} \leq 2^{5} D N_{k}^{\alpha}}\left\|\mathfrak{O}_{4,1}\left(\omega_{k, D}^{\alpha}, P_{N^{\prime}, M^{\prime}} f\right)\right\|_{N(I)} \lesssim \mu^{4}\left(\frac{N_{k}^{\alpha}}{N^{\prime}}+\frac{1}{N_{k}^{\alpha}}\right)^{\delta_{0} / 4}\left\|P_{N^{\prime}} \tilde{f}\right\|_{\tilde{X}_{\rho}^{1}(I)}
$$

which gives by (7.68) combined with (7.69) that the contribution of $P_{\geq 2^{10} D N_{k}^{\alpha}} f$ is

$$
\left\|F^{\prime}\left(\omega_{k, D}^{\alpha}\right) P \geq D N_{k}^{\alpha} f\right\|_{N(I)} \lesssim_{D, \theta} \mu^{3}\|f\|_{\widetilde{X}_{\rho}^{1}(I)} .
$$

This and (7.66) give the desired bound if $\mu \leq \mu_{0}(D, \theta)$, which finishes the proof of Lemma 7.8 .

We end this section by proving the following smoothing lemma:

Lemma 7.9. Suppose that $\psi \in H^{1}\left(\mathbb{R} \times \mathbb{T}^{2}\right)$ satisfies the following:

$$
\|\psi\|_{H^{1}\left(\mathbb{R} \times \mathbb{T}^{2}\right)} \lesssim 1 \quad\left\|e^{i t \Delta} \psi\right\|_{Z(\mathbb{R})} \leq \delta^{2}
$$

for some $0<\delta<1$. Then for any $R>0$, there exists $C(R) \geq 1$ such that:

$$
N\left\|\nabla^{1}\left(e^{i t \Delta} \tilde{P}_{\delta}^{1} \psi\right)\right\|_{L_{x, y, t}^{2}\left(\left\{\left|p-p_{0}\right| \leq R N^{-1}\right\} \times\left\{\left|t-t_{0}\right| \leq R N^{-2}\right\}\right)} \leq C(R) \delta^{\frac{1}{100}} .
$$

for any dyadic integer $N \geq 1$ and $t_{0} \in \mathbb{R}$ and any $p_{0} \in \mathbb{R} \times \mathbb{T}^{2}$.

Proof. Without any loss of generality, we may assume that $R=1, p_{0}=0$, and $t_{0}=0$ thanks to space and time translation invariance. From the definition of the $Z$-norm and (7.70), we have:

$$
\left\|e^{i t \Delta} P_{K} \psi\right\|_{L_{x, y, t}^{18}\left(\left\{|p| \leq N^{-1}\right\} \times\left\{|t| \leq N^{-2}\right\}\right)} \lesssim \delta^{2} K^{\frac{2}{9}},
$$

from which it follows by Hölder's inequality that:

$$
N\left\|\nabla^{1}\left(e^{i t \Delta} P_{K} \widetilde{P}_{\delta}^{1} \psi\right)\right\|_{L_{x, y, t}^{2}\left(\left\{|p| \leq N^{-1}\right\} \times\left\{|t| \leq N^{-2}\right\}\right)} \lesssim \delta^{2} N^{-\frac{11}{9}} K^{\frac{11}{9}} .
$$

On the other hand, estimating differently, we have from the smoothing estimate (7.39) that:

$$
\begin{aligned}
N\left\|\nabla^{1}\left(e^{i t \Delta} P_{K} P_{\delta}^{1} \psi\right)\right\|_{L_{x, y, t}^{2}\left(\left\{|p| \leq N^{-1}\right\} \times\left\{|t| \leq N^{-2}\right\}\right)} & \lesssim N^{1 / 2}\left\|\nabla^{1}\left(e^{i t \Delta} P_{K} P_{\delta}^{1} \psi\right)\right\|_{L_{x}^{\infty} L_{y, t}^{2}\left(\left\{|p| \leq N^{-1}\right\} \times\left\{|t| \leq N^{-2}\right\}\right)} \\
& \lesssim N^{1 / 2}(\delta K)^{-1 / 2} .
\end{aligned}
$$

\footnotetext{
${ }^{14}$ Notice that here, we use crucially the gain from [31, Proposition 3.5].
} 
Combining (7.72) and (17.73) and summing in $K$ yields (7.71).

\section{Appendix: Local Theory of the Resonant System}

In this appendix, we collect some facts about resonant quintic system (1.6). This system is the Hamiltonian flow of the positive definite Hamiltonian:

$$
H[\vec{u}]=\frac{1}{2} \sum_{q \in \mathbb{Z}^{2}} \int_{\mathbb{R}}\left|\partial_{x} u_{q}\right|^{2} d x+\frac{1}{6} \sum_{q \in \mathbb{Z}^{2}, n \in \mathbb{N}} \int_{\mathbb{R}}\left|\sum_{\begin{array}{c}
p_{1}-p_{2}+p_{3}=q \\
\left|p_{1}\right|^{2}-\left|p_{2}\right|^{2}+\left|p_{3}\right|^{2}=n
\end{array}} u_{p_{1}} \overline{u_{p_{2}}} u_{p_{3}}\right|^{2} d x .
$$

In addition, (1.6) enjoys the following conservation laws: for weights $g(p)=g_{a, b, c}(p)=a+b p+c|p|^{2}$, the solutions of (1.6) have formally conserved $g$-energy where,

$$
E_{g}(\vec{u})=\sum_{p \in \mathbb{Z}^{2}} g(p)\left\|u_{p}\right\|_{L^{2}(\mathbb{R})}^{2} .
$$

Indeed,

$$
\begin{aligned}
\frac{d}{d t} E_{g}(\vec{u}) & =\sum_{\substack{p_{0}+p_{2}+p_{4}=p_{1}+p_{3}+p_{5} \\
\left|p_{0}\right|^{2}+\left|p_{2}\right|^{2}+\left|p_{4}\right|^{2}=\left|p_{1}\right|^{2}+\left|p_{3}\right|^{2}+\left|p_{5}\right|^{2}}} g\left(p_{0}\right) \operatorname{Im}\left\{\int_{\mathbb{R}} \overline{u_{p_{0}} u_{p_{2}} u_{p_{4}}} u_{p_{1}} u_{p_{3}} u_{p_{5}} d x\right\} \\
& =\frac{1}{3} \sum_{\substack{p_{0}+p_{2}+p_{4}=p_{1}+p_{3}+p_{5} \\
\left|p_{0}\right|^{2}+\left|p_{2}\right|^{2}+\left|p_{4}\right|^{2}=\left|p_{1}\right|^{2}+\left|p_{3}\right|^{2}+\left|p_{5}\right|^{2}}}\left[g\left(p_{0}\right)+g\left(p_{2}\right)+g\left(p_{4}\right)\right] \operatorname{Im}\left\{\int_{\mathbb{R}} \overline{u_{p_{0}} u_{p_{2}} u_{p_{4}}} u_{p_{1}} u_{p_{3}} u_{p_{5}} d x\right\} \\
& =0 .
\end{aligned}
$$

The following proposition proves the local well-posedness and small-data scattering for the quintic system (1.6). We recall that the norm $\vec{W}(I)$ is defined as:

$$
\|\vec{u}\|_{\vec{W}(I)}^{2}:=\sum_{p \in \mathbb{Z}^{2}}\left[1+|p|^{2}\right]\left\|u_{p}\right\|_{L_{x, t}^{6}\left(\mathbb{R}_{x} \times I\right)}^{2} .
$$

Proposition 8.1 (Local well-posedness and small-data scattering for (1.6)). Let $\vec{u}(0)=\left\{u_{p}(0)\right\}_{p} \in h^{1} L^{2}$ satisfy $\left\|\vec{u}_{0}\right\|_{h^{1} L^{2}} \leq E$, then:

(1) there exists an open interval $0 \in I$ and a unique solution $\vec{u}(t)$ of (1.6) in $C_{t}^{0}\left(I: h^{1} L^{2}\right) \cap \vec{W}(I)$.

(2) there exists $E_{0}$ such that if $E \leq E_{0}, \vec{u}(t)$ is global and scatters in positive and negative infinite time.

(3) propagation of regularity: if $\vec{u}(0) \in h^{\sigma} H^{k}$ for some $\sigma \geq 1$ and $k \geq 0$, then $\vec{u} \in C_{t}^{0}\left(I: h^{\sigma} H^{k}\right)$.

Proof. The proof follows from a simple fixed point theorem (and classical arguments) once we have established the fundamental nonlinear estimate. By the scalar Strichartz estimates, we see that

$$
\left\|u_{j}\right\|_{L_{x, t}^{6}(\mathbb{R} \times I)} \lesssim\left\|u_{j}(0)\right\|_{L^{2}}+\sum_{\mathcal{R}(j)}\left\|u_{p_{1}} \overline{u_{p_{2}}} u_{p_{3}} \overline{u_{p_{4}}} u_{p_{5}}\right\|_{L_{x, t}^{\frac{6}{5}}(\mathbb{R} \times I)},
$$

where $\mathcal{R}(j)$ was defined in (1.6). Multiplying by $\langle j\rangle$ and square-summing, the first term on the right-hand side is bounded by the square of the $h^{1} L_{x}^{2}$-norm. For the second term, we compute using Lemma 8.2 
below that

$$
\begin{aligned}
& \sum_{j \in \mathbb{Z}^{2}}\langle j\rangle^{2}\left[\sum_{\mathcal{R}(j)}\left\|u_{p_{1}} \overline{u_{p_{2}}} u_{p_{3}} \overline{u_{p_{4}}} u_{p_{5}}\right\|_{L_{x, t}^{\frac{6}{5}}(\mathbb{R} \times I)}\right]^{2} \\
& \lesssim \sum_{j \in \mathbb{Z}^{2}}\langle j\rangle^{2}\left[\sum_{\mathcal{R}(j)} \Pi_{k=1}^{5}\left\|u_{p_{k}}\right\|_{L_{x, t}^{6}(\mathbb{R} \times I)}\right]^{2} \\
& \lesssim \sum_{j \in \mathbb{Z}^{2}}\left\{\sum_{\mathcal{R}(j)} \Pi_{k=1}^{5}\left\langle p_{k}\right\rangle^{2}\left\|u_{p_{k}}\right\|_{L_{x, t}^{6}(\mathbb{R} \times I)}^{2} \times\langle j\rangle^{2} \sum_{\mathcal{R}(j)}\left\langle p_{1}\right\rangle^{-2}\left\langle p_{2}\right\rangle^{-2}\left\langle p_{3}\right\rangle^{-2}\left\langle p_{4}\right\rangle^{-2}\left\langle p_{5}\right\rangle^{-2}\right\} \\
& \lesssim \sum_{j \in \mathbb{Z}^{2}} \sum_{\mathcal{R}(j)} \Pi_{k=1}^{5}\left\langle p_{k}\right\rangle^{2}\left\|u_{p_{k}}\right\|_{L_{x, t}^{6}(\mathbb{R} \times I)}^{2} \lesssim\|\vec{u}\|_{\vec{W}(I)}^{10} .
\end{aligned}
$$

Consequently, we obtain

$$
\|\vec{u}\|_{\vec{W}(I)} \lesssim\left\|e^{i t \partial_{x x}} \vec{u}_{0}\right\|_{\vec{W}(I)}+\|\vec{u}\|_{\vec{W}(I)}^{5} .
$$

This and the Strichatz estimate:

$$
\left\|e^{i t \partial_{x x}} \vec{u}_{0}\right\|_{\vec{W}(\mathbb{R})} \lesssim\left\|\vec{u}_{0}\right\|_{h^{1} L^{2}} \lesssim E
$$

allows one to run a classical fixed-point argument in $\vec{W}(I) \cap C_{t}\left(I: h^{1} L^{2}\right)$ provided $I$ or $E$ is small enough. The rest of the proposition follows from standard arguments.

Lemma 8.2. There holds that

$$
\sup _{j \in \mathbb{Z}^{2}}\left\{\langle j\rangle^{2} \sum_{\left(p_{1}, p_{2}, p_{3}, p_{4}, p_{5}\right) \in \mathcal{R}(j)}\left\langle p_{1}\right\rangle^{-2}\left\langle p_{2}\right\rangle^{-2}\left\langle p_{3}\right\rangle^{-2}\left\langle p_{4}\right\rangle^{-2}\left\langle p_{5}\right\rangle^{-2}\right\} \lesssim 1
$$

Proof. Without loss of generality, we may assume that

$$
\left|p_{1}\right| \leq\left|p_{3}\right| \leq\left|p_{5}\right|, \quad\left|p_{2}\right| \leq\left|p_{4}\right|, \quad \max \left(|j|,\left|p_{4}\right|\right) \sim\left|p_{5}\right|
$$

The first equation in the resonant condition specifies

$$
p_{5}=p_{2}+p_{4}+j-p_{1}-p_{3},
$$

while the second condition specifies that $p_{3}$ lies on a specific circle $\mathcal{C}$, namely

$$
\left|p_{3}-\frac{p_{2}+p_{4}+j-p_{1}}{2}\right|^{2}=\frac{2\left[\left|p_{2}\right|^{2}+\left|p_{4}\right|^{2}+|j|^{2}-\left|p_{1}\right|^{2}\right]-3\left|p_{2}+p_{4}+j-p_{1}\right|^{2}}{4}
$$

We can use Lemma 8.3 below to bound the number of possible $p_{3}$ :

$$
\sum_{p_{3} ;\left|p_{3}\right| \geq \max \left(\left|p_{1}\right|,\left|p_{2}\right|,\left|p_{4}\right|\right) ; p_{3} \in \mathcal{C}}\left\langle p_{3}\right\rangle^{-2} \lesssim\left\langle\max \left(\left|p_{1}\right|,\left|p_{2}\right|,\left|p_{4}\right|\right)\right\rangle^{-1}
$$


Using this, we obtain that

$$
\begin{aligned}
S_{1} & =\sum_{\substack{\left(p_{1}, p_{2}, p_{3}, p_{4}, p_{5}\right) \in \mathcal{R}(j) ; \\
\left|p_{2}\right| \leq\left|p_{4}\right| \leq\left|p_{3}\right| ;\left|p_{1}\right| \leq\left|p_{3}\right| \leq\left|p_{5}\right|}}\left\langle p_{1}\right\rangle^{-2}\left\langle p_{2}\right\rangle^{-2}\left\langle p_{3}\right\rangle^{-2}\left\langle p_{4}\right\rangle^{-2} \frac{\langle j\rangle^{2}}{\left\langle p_{5}\right\rangle^{2}} \\
& \lesssim \sum_{p_{1}, p_{2}, p_{4}}\left\langle p_{1}\right\rangle^{-2}\left\langle p_{2}\right\rangle^{-2}\left\langle p_{4}\right\rangle^{-2} \sum_{\substack{p_{3}:\left(p_{1}, p_{2}, p_{3}, p_{4}, p_{2}+p_{4}+j-p_{1}-p_{3}\right) \in \mathcal{R}(j),\left|p_{3}\right| \geq \max \left(\left|p_{1}\right|,\left|p_{2}\right|,\left|p_{4}\right|\right)}}\left\langle p_{3}\right\rangle^{-2} \\
& \lesssim \sum_{p_{1}, p_{2}, p_{4}}\left\langle p_{1}\right\rangle^{-2}\left\langle p_{2}\right\rangle^{-2}\left\langle p_{4}\right\rangle^{-2}\left\langle\left|p_{1}\right|+\left|p_{2}\right|+\left|p_{4}\right|\right\rangle^{-1} \lesssim 1 .
\end{aligned}
$$

The sum when $\left|p_{3}\right| \leq\left|p_{4}\right|$ is bounded similarly, using Lemma 8.3 to bound the sum over $p_{4}$ instead of the bound over $p_{3}$.

We are left with

Lemma 8.3. For any $P \in \mathbb{R}^{2}, R>0$ and $A>1$ there holds that:

$$
\sum_{\substack{|p| \geq A \\ p \in \mathbb{Z}^{2} \cap C(P, R)}} \frac{1}{\langle p\rangle^{2}} \lesssim A^{-1},
$$

where $C(P, R)$ denotes the circle of radius $R$ centered at $P$.

Proof. Indeed, it suffices to prove that for any $k \geq 0$,

$$
\left|\left\{p:|p| \in\left[2^{k} A, 2^{k+1} A\right] ; p \in C(P, R)\right\}\right| \lesssim 2^{k} A .
$$

When $R \leq 2^{k+10} A$, this follows directly from the fact that the integer points are 1-separated and hence there can be at most $2 \pi R$ points on a circle of radius $R$. When $R \geq 2^{k+10} A$, we have that

$$
\left|D\left(0,2^{k+1} A\right) \cap C(P, R)\right| \lesssim 2^{k} A,
$$

where $D(0, K)$ denotes the disc centered at the origin of radius $K$. To see this, let $\alpha$ be the angle of the above arc in $C(P, R)$. This arc has length $R \alpha$ while from the fact that the chord has to fit into the disc, we also obtain that $R \tan \alpha \leq 2^{k+2} A$ which gives the result.

One can also prove by standard arguments a stability result for (1.6) similar to Lemma 4.6, which will allow to prove:

Lemma 8.4. Assume the conclusion of Theorem 1.3 holds for all initial data $u_{0} \in H^{1}\left(\mathbb{R} \times \mathbb{T}^{2}\right)$ with full energy $L\left(u_{0}\right)<E_{\max }$. Then Conjecture 1.2 holds true for all initial data $\vec{v}_{0} \in h^{1} L^{2}$ satisfying $E_{l s}\left(\vec{v}_{0}\right)<E_{\text {max }}$. In particular, if all finite full-energy (i.e. $H^{1}$ ) solutions scatter for (1.1), then the same holds for finite $E_{l s}$-energy solutions of (1.6).

\section{REFERENCES}

[1] M. J. Ablowitz, B. Prinari and A. D. Trubatch, Discrete and Continuous Nonlinear Schrödinger Systems, London Mathematical Society Lecture Note Series (No. 302), Cambridge University Press, ISBN: 9780521534376.

[2] T. Alazard and R. Carles, Loss of regularity for supercritical nonlinear Schrödinger equations. Math. Ann. 343 (2009), no. 2, 397-420.

[3] H. Bahouri and P. Gérard, High frequency approximation of solutions to critical nonlinear wave equations. Amer. J. Math. 121 (1999), no. 1, 131-175.

[4] V. Banica, R. Carles and T. Duyckaerts, On scattering for NLS: from Euclidean to hyperbolic space, Discrete Contin. Dyn. Syst. 24 (2009), 1113-1127. 
[5] P. Bégout and A. Vargas, Mass concentration phenomena for the $L^{2}$-critical nonlinear Schrödinger equation, Trans. Am. Math. Soc., 359 (11), (2007), 5257-5282.

[6] J.M. Bouclet, Strichartz estimates on asymptotically hyperbolic manifolds. Anal. PDE 4 (2011), no. 1, 1-84.

[7] J. M. Bouclet and N. Tzvetkov, Strichartz estimates for long range perturbations. Amer. J. Math. 129 (2007), no. 6 , $1565-1609$.

[8] J. Bourgain, Fourier transform restriction phenomena for certain lattice subsets and applications to nonlinear evolution equations. I. Schrödinger equations, Geom. Funct. Anal. 3 (1993), 107-156.

[9] J. Bourgain, Exponential sums and nonlinear Schrödinger equations, Geom. Funct. Anal. 3 (1993), 157-178.

[10] J. Bourgain, Global wellposedness of defocusing critical nonlinear Schrödinger equation in the radial case, J. Amer. Math. Soc. 12 (1999), 145-171.

[11] H. Brézis and T. Gallouët, Nonlinear Schrödinger evolution equations. Nonlinear Anal., Theory Methods Appl. 4, 677-681 (1980).

[12] N. Burq, P. Gérard, and N. Tzvetkov, Strichartz inequalities and the nonlinear Schrödinger equation on compact manifolds, Amer. J. Math. 126 (2004), 569-605.

[13] N. Burq, P. Gérard and N. Tzvetkov, Multilinear eigenfunction estimates and global existence for the three dimensional nonlinear Schrödinger equations. Ann. Sci. École Norm. Sup. 38 (2005), 255-301.

[14] N. Burq, P. Gérard, and N. Tzvetkov, Bilinear eigenfunction estimates and the nonlinear Schrödinger equation on surfaces, Invent. Math. 159 (2005), 187-223.

[15] R. Carles, and S. Keraani, On the role of quadratic oscillations in the nonlinear Schrödinger equation II, the $L^{2}$-critical case, Trans. Amer. Math. Soc. 359 (2007), 33-62.

[16] T. Cazenave, Semilinear Schrödinger Equations, Courant Lecture Notes in Mathematics 10, New York University, Courant Institute of Mathematical Sciences, New York; American Mathematical Society, Providence, RI, 2003.

[17] J. Coleman, Blowup phenomena for the vector nonlinear Schrödinger equation, PhD thesis, U. Toronto, ISBN 10: 0612636941

[18] J. Colliander, M. Keel, G. Staffilani, H. Takaoka and T. Tao, Global well-posedness and scattering for the energy-critical nonlinear Schrödinger equation in $\mathbb{R}^{3}$, Ann. of Math. 167 (2008), 767-865.

[19] J. Colliander, M. Keel, G. Staffilani, H. Takaoka and T. Tao, Transfer of energy to high frequencies in the cubic defocusing nonlinear Schrödinger equation, Invent. Math. 181 (2010), 39-113.

[20] B. Dodson, Global well-posedness and scattering for the defocusing, $L^{2}$-critical, nonlinear Schrödinger equation when $d \geq 3$, preprint, arXiv:0912.2467

[21] B. Dodson, Global well-posedness and scattering for the defocusing, $L^{2}$-critical, nonlinear Schrödinger equation when $d=1$, preprint, arXiv:1010.0040

[22] David Duchesne, Marcello Ferrera, Luca Razzari, Roberto Morandotti, Brent Little, Sai T. Chu and David J. Moss, Nonlinear Optics in Doped Silica Glass Integrated Waveguide Structures, Frontiers in Guided Wave Optics and Optoelectronics, Bishnu Pal (Ed.), (2010), ISBN: 978-953-7619-82-4.

[23] T. Duyckaerts, J. Holmer, and S. Roudenko, Scattering for the non-radial 3D cubic nonlinear Schrödinger equation, Math. Res. Lett. 15 (2008), no. 6, 1233-1250.

[24] P. Gérard and V. Pierfelice, Nonlinear Schrödinger equation on four-dimensional compact manifolds, Bull. Soc. Math. France 138 (2010), 119-151.

[25] V. S. Gerdjikov, On Soliton Interactions of Vector Nonlinear Schrödinger Equations AIP Conf. Proc. 1404, 57 (2011).

[26] B. Grebert and L. Thomann, Resonant dynamics for the quintic nonlinear Schrödinger equation, Preprint.

[27] M. Grillakis, On nonlinear Schrödinger equations, Comm. Partial Differential Equations 25 (2000), $1827-1844$.

[28] M. Hadac, S. Herr and H. Koch, Well-posedness and scattering for the KP-II equation in a critical space. Ann. Inst. H. Poincaré Anal. Non Linéaire 26 (2009), no. 3, 917-941.

[29] Z. Hani, Global well-posedness of the cubic nonlinear Schrödinger equation on compact manifolds without boundary, Comm. Partial Differential Equations, to appear.

[30] S. Herr, The quintic nonlinear Schrödinger equation on three-dimensional Zoll manifolds, Amer. J. Math. to appear, arXiv:1101.4565

[31] S. Herr, D. Tataru, and N. Tzvetkov, Global well-posedness of the energy critical nonlinear Schrödinger equation with small initial data in $H^{1}\left(\mathbb{T}^{3}\right)$, Duke Math. J. 159 (2011), 329-349.

[32] S. Herr, D. Tataru, and N. Tzvetkov, Strichartz estimates for partially periodic solutions to Schrödinger equations in $4 d$ and applications, Preprint, arXiv:1011.0591.

[33] A. D. Ionescu, B. Pausader, and G. Staffilani, On the global well-posedness of energy-critical Schrödinger equations in curved spaces, Analysis and PDE, to appear, arXiv:1008.1237.

[34] A. D. Ionescu and B. Pausader, Global wellposedness of the energy-critical defocusing NLS on $\mathbb{R} \times \mathbb{T}^{3}$, Comm. Math. Phys., to appear, arXiv:1101.4527

[35] A. D. Ionescu and B. Pausader, The energy-critical defocusing NLS on $\mathbb{T}^{3}$, Duke Math. J., to appear. 
[36] C. E. Kenig and F. Merle, Global well-posedness, scattering and blow-up for the energy-critical, focusing, non-linear Schrödinger equation in the radial case, Invent. Math. 166 (2006), 645-675.

[37] C. E. Kenig and F. Merle, Global well-posedness, scattering and blow-up for the energy-critical focusing non-linear wave equation, Acta Math. 201 (2008), 147-212.

[38] S. Keraani, On the defect of compactness for the Strichartz estimates of the Schrödinger equations, J. Diff. Equ. 175 (2001), 353-392.

[39] R. Killip, S. Kwon, S. Shao and M. Visan, On the mass-critical generalized KdV equation, DCDS-A 32 (2012), 191-221.

[40] R. Killip, B. Stovall and M. Visan, Scattering for the cubic Klein-Gordon equation in two space dimensions, Trans. Amer. Math. Soc. 364 (2012), 1571-1631.

[41] R. Killip, T. Tao and M. Visan, The cubic nonlinear Schrödinger equation in two dimensions with radial data, J. Eur. Math. Soc. 11 (2009), no. 6, 1203-1258.

[42] R. Killip and M. Visan, Global well-posedness and scattering for the defocusing quintic NLS in three dimensions, Analysis and PDE, to appear, arXiv:1102.1192

[43] Q. Lin, Oskar J. Painter, and Govind P. Agrawal, Nonlinear optical phenomena in silicon waveguides: Modeling and applications, Optics Express, Vol. 15, Issue 25 (2007), p. 16604-16644.

[44] J. Metcalfe, C. D. Sogge, and A. Stewart: Nonlinear hyperbolic equations in infinite homogeneous waveguides. Comm. Partial Differential Equations 30 (2005), 643-661.

[45] B. Pausader, Scattering for the Beam Equation in Low Dimensions, Indiana Univ. Math. J., 59 (2010), no. 3, 791-822.

[46] T. Roy, Scattering above energy norm of solutions of a loglog energy-supercritical Schrödinger equation with radial data. J. Diff. Equ. 250 (2011), no. 1, 292-319.

[47] T. Schneider, Nonlinear Optics in Telecommunications (Advanced Texts in Physics), Springer, 1rst edition, 2004. ISBN 3540201955.

[48] C. Sulem and P.L. Sulem, The nonlinear Schrödinger equation. Self-focusing and wave collapse, Chapter 13. Applied Mathematical Sciences, 139. Springer-Verlag, New York, 1999. ISBN: 0-387-98611-1.

[49] H. Takaoka and N. Tzvetkov, On $2 D$ Nonlinear Schrödinger equations with data on $\mathbb{R} \times \mathbb{T}$, J. Funct. Anal. 182 (2001), $427-442$

[50] T. Tao, Nonlinear Dispersive Equations, Local and Global Analysis, CBMS Regional Conference Series in Mathematics, 106, American Mathematical Society, Providence, RI, 2006.

[51] T. Tao, A pseudoconformal compactification of the nonlinear Schrödinger equation and applications, New York J. Math. 15 (2009), 265-282.

[52] T. Tao, Global regularity for a logarithmically supercritical defocusing nonlinear wave equation for spherically symmetric data, J. Hyperbolic Diff. Eq. 4 (2007), 259-266.

[53] T. Tao, M. Visan and X. Zhang, Minimal-mass blowup solutions of the mass-critical NLS, Forum Math. 20 (2008), 881-919.

[54] L. Thomann, Instabilities for supercritical Schrödinger equations in analytic manifolds. J. Diff. Equ. 245 (2008), no. $1,249-280$.

[55] N. Tzvetkov, N. Visciglia, Small data scattering for the nonlinear Schrödinger equation on product spaces, Preprint, arXiv:1011.6185.

Courant Institute of Mathematical Sciences, 251 Mercer Street, New York NY 10012

E-mail address: hani@cims.nyu.edu

E-mail address: pausader@cims.nyu.edu 